Florida International University FIU Digital Commons

$3-27-2013$

\title{
Localized Surface Plasmon Resonance Biosensors for Real-Time Biomolecular Binding Study
}

Chang Liu

Florida International University, cliu003@fiu.edu

DOI: $10.25148 /$ etd.FI13042210

Follow this and additional works at: https://digitalcommons.fiu.edu/etd

\section{Recommended Citation}

Liu, Chang, "Localized Surface Plasmon Resonance Biosensors for Real-Time Biomolecular Binding Study" (2013). FIU Electronic Theses and Dissertations. 837.

https://digitalcommons.fiu.edu/etd/837

This work is brought to you for free and open access by the University Graduate School at FIU Digital Commons. It has been accepted for inclusion in FIU Electronic Theses and Dissertations by an authorized administrator of FIU Digital Commons. For more information, please contact dcc@fiu.edu. 


\section{FLORIDA INTERNATIONAL UNIVERSITY}

Miami, Florida

\section{LOCALIZED SURFACE PLASMON RESONANCE BIOSENSORS FOR REAL-TIME BIOMOLECULAR BINDING STUDY}

A dissertation submitted in partial fulfillment of the requirements for the degree of DOCTOR OF PHILOSOPHY

in

BIOMEDICAL ENGINEERING

by

Chang Liu

2013 
To: Dean Amir Mirmiran

College of Engineering and Computing

This dissertation, written by Chang Liu, and entitled Localized Surface Plasmon Resonance Biosensors for Real-Time Biomolecular Binding Study, having been approved in respect to style and intellectual content, is referred to you for judgment.

We have read this dissertation and recommend that it be approved.

Wei-Chiang Lin

Anuradha Godavarty

Barry Rosen

Chenzhong Li, Major Professor

Date of Defense: March 26, 2013

The dissertation of Chang Liu is approved.

Dean Amir Mirmiran

College of Engineering and Computing

Dean Lakshmi N. Reddi

University Graduate School

Florida International University, 2013 


\section{DEDICATION}

I would like to dedicate this dissertation to my family whose love and support are the driving force of my life. 


\section{ACKNOWLEDGMENTS}

This dissertation has been made possible by the collective support of my family, friends and mentors. I would like to thank my wife, my parents, my grandparents and my aunts who are my greatest support system in life. Their unconditional love and support have kept me going through the hard times. My mentor Dr. Chenzhong Li has taught me a great deal and will be a father-figure for life. He has always had my best interest at heart and has provided me with every opportunity to grow. I am grateful for all the kindness and patience that he has shown me over the years. I would also like to thank my dissertation committee members Dr. Anuradha Godavarty, Dr. Wei-Chiang Lin and Dr. Barry Rosen for their invaluable advice in steering me towards the right direction. My lab members Dr. Subbiah Alwarappan, Shradha Prabhulkar, Evangelia Hondroulis, Rakesh Guduru, Pratikkumar Shah, Xuena Zhu, Dali Sun and Qiang Shi have been great friends as well as colleagues and have helped me not just with academics but to grow as a person.

The friends I made in Miami have been my surrogate family and have helped me on every step of the way. They have come and gone but each one of them has touched my life in a special way and taught me to live life to the fullest. Their support and good wishes have been the driving force in helping me successfully finish my dissertation.

I would also like to acknowledge FIU Graduate School Doctoral Evidence Acquisition Fellowship and Chinese Government Award for Outstanding Self-financed Students Aboard for financial supports through my doctoral study. I also thank AMERI (Advanced Material Engineering Research Institute, FIU) for technical support. This work has also been partially supported by U.S. Public Health Service Grant GM55425 to 
Professor Barry Rosen, NIH R15 ES021079-01 and W81XWH-10-1-0732 by U.S. Army Medical Research \& Material Command (USAMRMC) and the Telemedicine \& Advanced Technology Research Center (TATRC) to Professor Chenzhong Li, Everglades Foundation and Kauffman Foundations.

I truly thank all of you from the bottom of my heart. 
ABSTRACT OF THE DISSERTATION

LOCALIZED SURFACE PLASMON RESONANCE BIOSENSORS FOR REAL-TIME

BIOMOLECULAR BINDING STUDY

by

Chang Liu

Florida International University, 2013

Miami, Florida

\section{Professor Chenzhong Li, Major Professor}

Surface Plasmon Resonance (SPR) and localized surface plasmon resonance (LSPR) biosensors have brought a revolutionary change to in vitro study of biological and biochemical processes due to its ability to measure extremely small changes in surface refractive index (RI), binding equilibrium and kinetics. Strategies based on LSPR have been employed to enhance the sensitivity for a variety of applications, such as diagnosis of diseases, environmental analysis, food safety, and chemical threat detection. In LSPR spectroscopy, absorption and scattering of light are greatly enhanced at frequencies that excite the LSPR, resulting in a characteristic extinction spectrum that depends on the RI of the surrounding medium. Compositional and conformational change within the surrounding medium near the sensing surface could therefore be detected as shifts in the extinction spectrum.

This dissertation specifically focuses on the development and evaluation of highly sensitive LSPR biosensors for in situ study of biomolecular binding process by incorporating nanotechnology. Compared to traditional methods for biomolecular binding studies, LSPR-based biosensors offer real-time, label free detection. First, we 
modified the gold sensing surface of LSPR-based biosensors using nanomaterials such as gold nanoparticles (AuNPs) and polymer to enhance surface absorption and sensitivity. The performance of this type of biosensors was evaluated on the application of small heavy metal molecule binding affinity study. This biosensor exhibited $\sim 7$ fold sensitivity enhancement and binding kinetics measurement capability comparing to traditional biosensors. Second, a miniaturized cell culture system was integrated into the LSPRbased biosensor system for the purpose of real-time biomarker signaling pathway studies and drug efficacy studies with living cells. To the best of our knowledge, this is the first LSPR-based sensing platform with the capability of living cell studies. We demonstrated the living cell measurement ability by studying the VEGF signaling pathway in living SKOV-3 cells. Results have shown that the VEGF secretion level from SKOV-3 cells is $0.0137 \pm 0.0012 \mathrm{pg}$ per cell. Moreover, we have demonstrated bevacizumab drug regulation to the VEGF signaling pathway using this biosensor. This sensing platform could potentially help studying biomolecular binding kinetics which elucidates the underlying mechanisms of biotransportation and drug delivery. 


\section{TABLE OF CONTENTS}

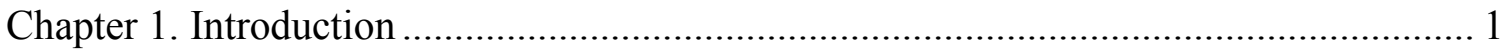

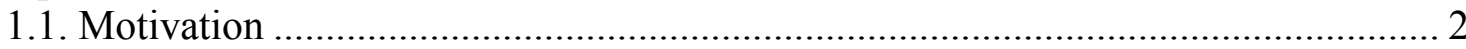

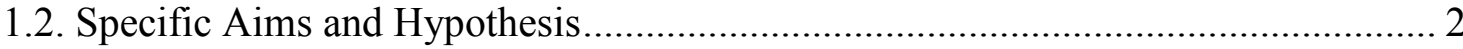

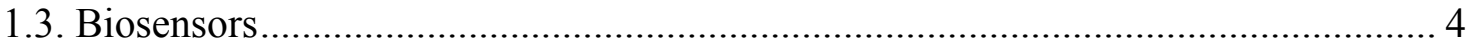

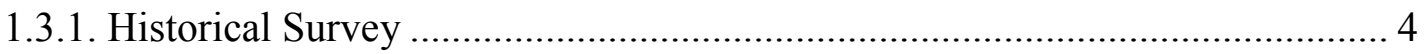

1.3.2. Definition and classification of biosensor.................................................... 5

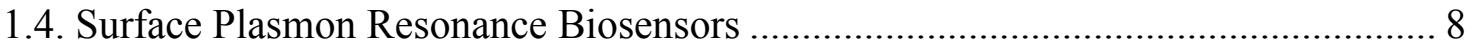

1.5. Other Methods for Binding Measurements ....................................................... 14

1.5.1. Current Methods to Measure Protein-Arsenic binding .................................... 14

1.5.2. Current Methods to Measure Biomarkers.................................................... 14

1.6. Polymer Brushes for Biosensing Signal Enhancement ....................................... 16

1.7. Arsenic Detoxification Research ......................................................................... 17

1.8. Current Chemotherapeutic Therapy and VEGF Regulation Therapy .................... 19

Chapter 2. Development of the highly sensitive LSPR sensor

and its application on the detection of small heavy metal molecule binding ................... 22

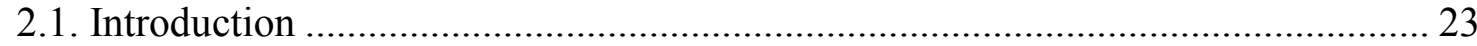

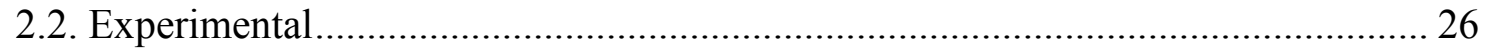

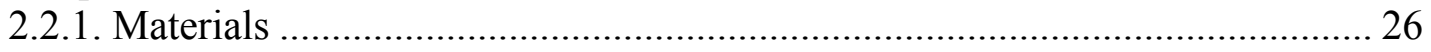

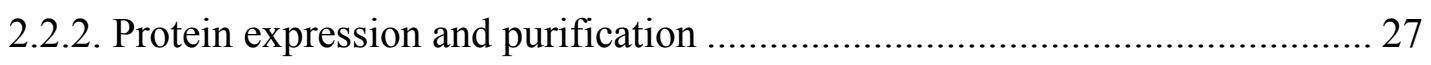

2.2.3. Preparation of gold nanoparticles ........................................................... 28

2.2.4. In situ polymerization .......................................................................... 28

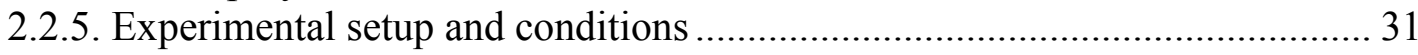

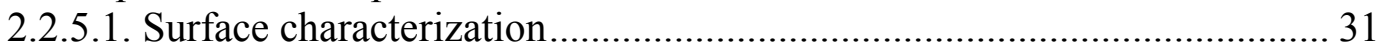

2.2.5.2. Preparation of LSPR measurement........................................................ 32

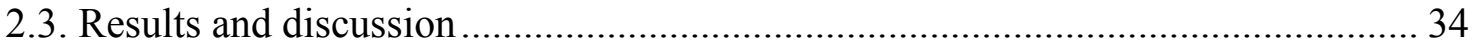

2.3.1. Surface morphology and composition characterization.................................. 34

2.3.2. Optimization of ArsA immobilization conditions .......................................... 36

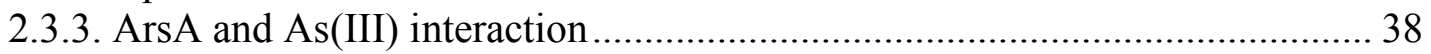

2.3.4. Kinetics study of ArsA-As(III) binding ......................................................... 40

2.3.5. Effects of ArsD and MgATP on ArsA-As(III) binding ................................ 42

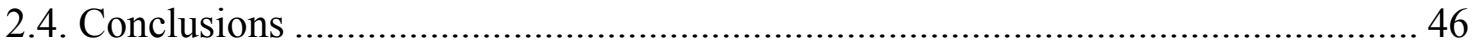

Chapter 3. Sensing platform upgrade for biomarker studies from a living cell model..... 48

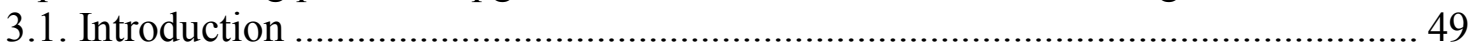

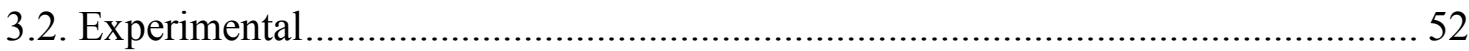

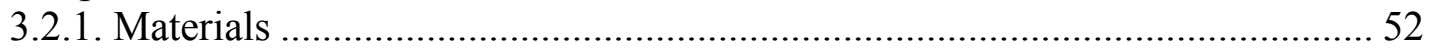

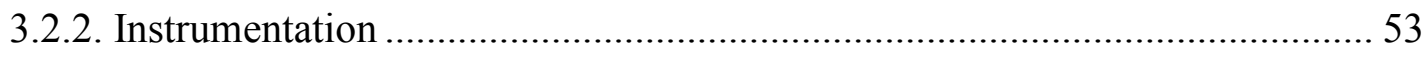

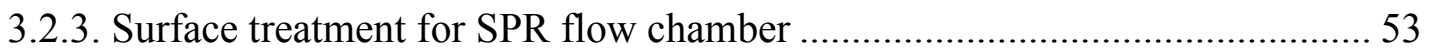

3.2.4. Human cell culture .................................................................................... 54

3.2.5. Cell stain and fluorescent imaging............................................................ 54 
3.2.6. Calibration of SPR for VEGF measurements ............................................... 55

3.2.7. Direct measurement of VEGF from living cancer cells.................................. 56

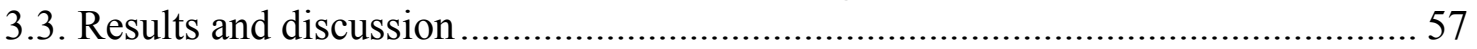

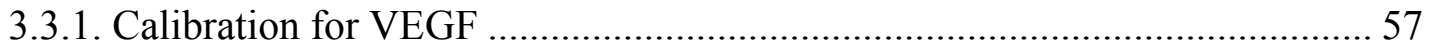

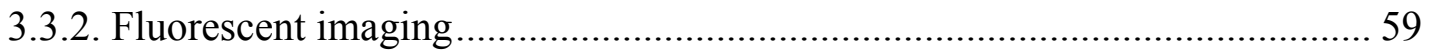

3.3.3. Direct measurement of VEGF from living cancer cells.....................................6 60

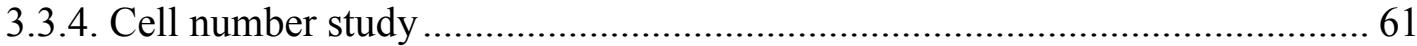

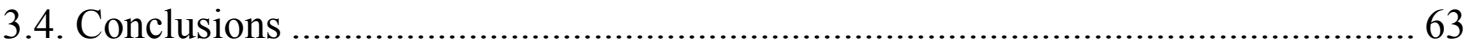

Chapter 4. Study of drug regulation on the VEGF binding kinetics with a living cell

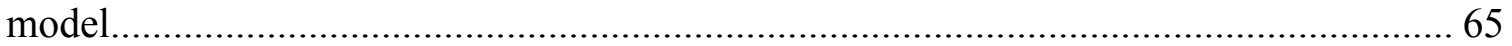

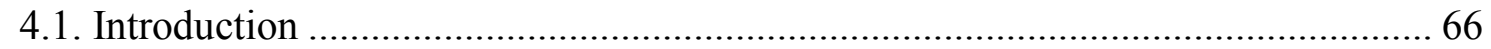

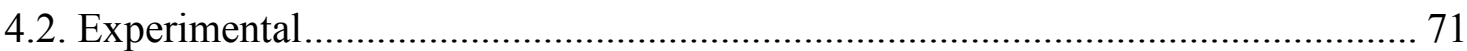

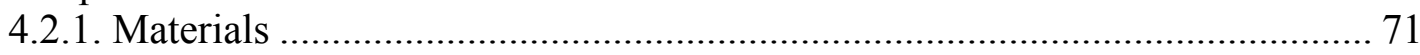

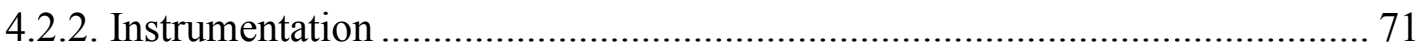

4.2.3. SPR flow chamber surface pre-treatment and human cell culture................... 72

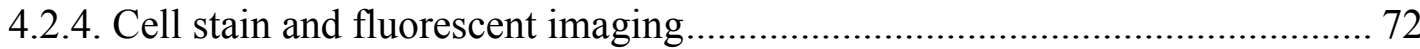

4.2.5. Preparation of SPR gold sensing surface ..................................................... 73

4.2.6. Comparison of VEGF-VEGFR and VEGF-bevacizumab binding kinetics ... 74

4.2.7. Drug regulation study on VEGF-VEGFR binding ........................................ 74

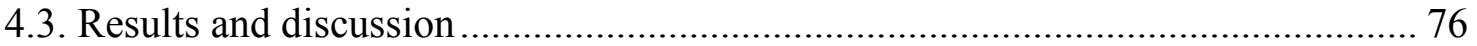

4.3.1. Optimization and calibration for surface concentration of bevacizumab ....... 76

4.3.2. Specificity study of VEGF-bevacizumab binding ........................................ 78

4.3.3. Study of SKOV-3 cell viability in the SPR flow chamber .............................. 81

4.3.4. Binding kinetics study of VEGF-VEGFR and VEGF-bevacizumab

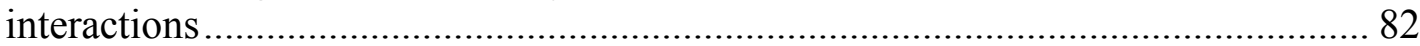

4.3.5. Bevacizumab drug regulation study on VEGF-VEGFR interaction................ 84

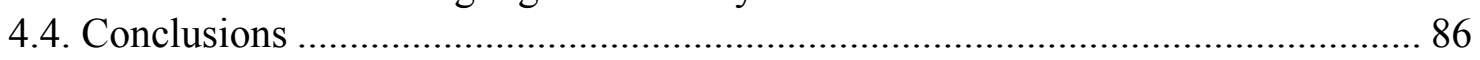

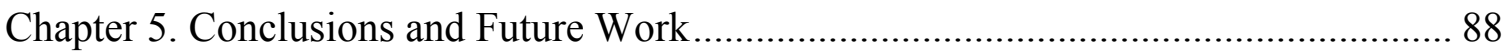

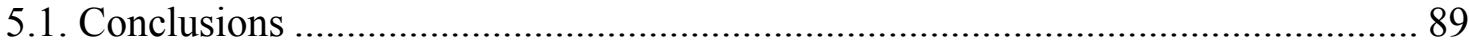

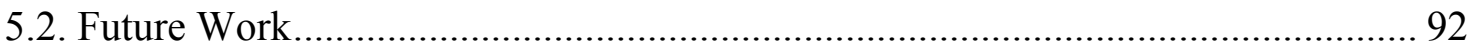

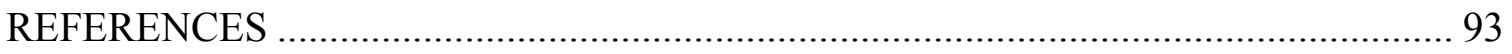

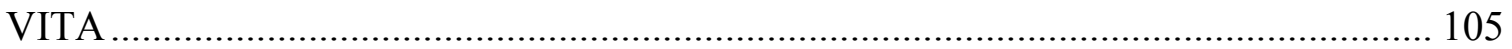




\section{LIST OF FIGURES}

FIGURE

PAGE

Figure 1.1. A schematic illustration of the main components of biosensors. ..............7

Figure 1.2. (a) Kretschmann configuration SPR, (b) Plasmon propagation parallel along the metal/dielectric interface. 9

Figure 1.3. Illustration of p-polarized light made incident on a planar interface of two medium. For a typical SPR instrument, medium 1 is a dielectric material such as glass, medium 2 is a metal such as gold. ....................................................................... 10

Figure 1.4. A picture of the BI-2000 flow injection SPR instrument. ...........................12

Figure 1.5. The sensorgram of baseline, association, dissociation and regeneration processes during a typical SPR experiment. .13

Scheme 2.1. (A) Cartoon representation of the swelling-shrinking process of the copolymer for LSPR signal amplification and immobilization of ArsA ATPase. (B) Structural illustration of the in situ radical copolymerization of PNIPAAm-co-PAAc hydrogel. .30

Figure 2.1. Experimental setup of the in situ radical copolymerization. 31

Figure 2.2. FTIR-ATR spectrum of the 3D PNIPAAm-co-PAAc polymer matrix on the sensing surface. .35

Figure 2.3. (A) AFM images of the polymer matrices under hydrated (upper) and dehydrated (lower) conditions, indicating the swelling-shrinking process. (B) SEM image of the LSPR sensor coated with AuNPs doped hydrogel thin film. .36

Figure 2.4. Optimization of immobilization conditions: (A) ArsA immobilization on the $3 \mathrm{D}$ polymer coated surface with different injection rate $(5,10,15,20,25,30,35 \mu \mathrm{L} / \mathrm{min})$. Maximum SPR angle shift is plotted versus responding injection rate. (B) Different concentration of ArsA $(10,20,30,40,50,60,70,80 \mu \mathrm{g} / \mathrm{mL})$ immobilization on the 3D polymer coated LSPR sensor with optimal injection rate $(20 \mu \mathrm{L} / \mathrm{min})$. Maximum SPR angle shift is plotted versus ArsA concentration. .38

Figure 2.5. SPR sensorgrams for the covalently immobilized ArsA interacting with As(III) obtained with (a) 3D AuNPs doped polymer coated sensor and (b) 2D AuNPs structure modifiedsensor. 39

Figure 2.6. SPR sensorgrams for covalently immobilized ArsA interacting with (red line) ArsD and (black line) BSA as control. .41

Figure 2.7. Comparison of ArsA-As(III) association rate constant (slope) under the effect 
of different binding status. Figure showing: linear fitting curve of the relationship between maximum binding rate and As(III) concentration under (A) noncatalytic, (B) ArsD bounded and (C) MgATP catalyzing conditions of ArsA. .44-46

Scheme 3.1 Configuration of the SPR based biosensor integrating a mini cell culture module for direct measurement of biomarker from living cells. .52

Figure 3.1. SPR Flow Cell and Flow Chamber Gasket. 54

Figure 3.2. (A: upper) SPR sensor responses to VEGF solution at different concentration levels: (a) at $0.25 \mu \mathrm{g} / \mathrm{mL}$, (b) at $0.5 \mu \mathrm{g} \mathrm{mL}$, (c) at $1 \mu \mathrm{g} / \mathrm{mL}$, (d) at $1.5 \mu \mathrm{g} / \mathrm{mL}$, (e) at $2 \mu \mathrm{g} / \mathrm{mL}$ and (f) at $2.5 \mu \mathrm{g} / \mathrm{mL}$. (B: lower) Calibration curve: the linear relationship between change of SPR angle shift and VEGF concentration. .58

Figure 3.3. Fluorescence images of SKOV-3 cell culture on: (upper) petri dish, (middle) gelatin coated PDMS and (lower) uncoated PDMS. $59-60$

Figure 3.4. SPR sensor responses to living SKOV-3 cells secretion stimulated by injections of $500 \mu \mathrm{m} \mathrm{Ca}^{2+}$ ionophore (A23187). Each arrow indicates an injection of $\mathrm{Ca}^{2+}$ ionophore.

Figure 3.5. SPR sensor responses to stimulated VEGF secretion from groups of cells with different cell numbers. VEGF secretion from (a) 80000 cells induced $43.4 \mathrm{mDeg}$ SPR angle shift; (b) 160000 cells induced $100.6 \mathrm{mDeg}$ SPR angle shift; (c) 320000 cells induced $214.2 \mathrm{mDeg}$ SPR angle shift. 63

Scheme 4.1. Schematic diagram of the relationships between VEGF and VEGF receptors. 68

Scheme 4.2. Schematic illustration of the biomimic system for bevacizumab drug regulation study. .75

Figure 4.1. SPR response to $3 \mu \mathrm{g} / \mathrm{mL}$ VEGF for each surface bound bevacizumab concentration: $5 \mu \mathrm{g} / \mathrm{mL}$ (red), $10 \mu \mathrm{g} / \mathrm{mL}$ (blue), $30 \mu \mathrm{g} / \mathrm{mL}$ (purple), $50 \mu \mathrm{g} / \mathrm{mL}$ (green). ....77

Figure 4.2. Calibration curve: the linear relationship between SPR baseline shift and the surface concentration of bevacizumab. .78

Figure 4.3. (A) SPR sensorgram of surface bound bevacizumab interacting with VEGF. Inset: enlarged sensorgram of the response upon VEGF binding. 80

Figure 4.3. (B) SPR sensorgram of surface bound bevacizumab interacting with VEGFR. Inset: enlarged sensorgram of the response upon VEGFR binding. .81

Figure 4.4. Fluorescent images of SKOV-3 cells at Hoechst channel, MitoTracker Red channel and merged images on different type of substrates: petri dishs (exposure time $400 \mathrm{~ms}$ ), gelatin coated gaskets (exposure time 200ms) and uncoated gaskets (exposure 
time $200 \mathrm{~ms})$

Figure 4.5. SPR sensorgram of VEGF binding response to bevacizumab (blue line) and VEGFR (red line).

Figure 4.6. SPR response of VEGF (SKOV-3 cells released) - VEGFR interaction (red line) and VEGF (SKOV-3 cells released) - VEGFR interaction under bevacizumab regulation (blue line) in the biomimic system. .86 


\section{ABBREVIATIONS AND ACRONYMS}

AAc: Acrylic acid

AFM: Atomic force microscope

AIBN: 2,2'azobisisobutyronitrile

ATP: Adenosine-5'-triphosphate

ATR-FTIR: Fourier transform infrared spectroscopy by attenuated total reflectance

AuNPs: Gold nanoparticles

BIS: N,N-methylenebisacrylamide

BSA: Bovine serum albumin

DIFP: Diisopropylfluorophosphate

DMA: Dimethylarsinic acid

DMSO: Dimethyl sulfoxide

DTT: Dithiothreitol

E. coli: Escherichia coli

EDC: 1-ethyl-3-(3-dimethylaminopropyl) carbodiimide hydrochloride

EDTA: Ethylenediaminetetraacetic acid

ELISA: Enzyme-linked immunosorbent assay

EXAFS: Extended x-ray absorption fine structure

$\operatorname{IgG}_{1}$ : Immunoglobulin $\mathrm{G}_{1}$

LB: Lysogeny broth

LSPR: Localized surface plasmon resonance

MgATP: Adenosine-5'-triphosphate and magnesium ion

MMA: Monomethylarsonic acid 
MOPS: 3-(N-morpholino)propanesulfonic acid

MUA: 11- mercaptoundecanoicacid

NBSs: Nucleotide binding sites

NCI: National cancer institute

NHS: N-Hydroxysuccinamide

NIH: National institutes of health

NIPAAm: N-isopropylacrylamide

PAAc: Poly (acrylic acid)

PBS: Phosphate buffered saline

PDMS: Polydimethylsiloxane

PNIPAAm: Poly (N-isopropylacrylamide)

PVP: Poly (vinyl pyridine)

RI: Refractive index

SAM: Self-assembled monolayer

SDS-PAGE: Sodium dodecyl sulfate polyacrylamide gel electrophoresis

SEM: Scanning electron microscopy

SIP: Surface initiated polymerization

SKOV-3: Human ovarian carcinoma cell line

SMP: Surface mediated polymerizations

SPR: Surface plasmon resonance

TNT: Trinitrotoluene

TSP: Thrombospondin

VEGF: Vascular endothelial growth factor 
VEGFR: Vascular endothelial growth factor receptor 
Chapter 1

Introduction 


\subsection{Motivation}

The motivation of this dissertation was to develop localized surface plasmon resonance (LSPR) sensors with enhanced sensitivity and capability of real-time kinetics measurements. Highly sensitive LSPR allows monitoring conformational change of proteins, binding affinity as well as binding kinetics bwtween two organic or inorganic molecules. This fundamental research could potentially benefit early diagnosis and drug development. SPR was used as the platform technology as it provides several key advantages such as high sensitivity, fast response time and label-free detection for biomolecular binding measurements. The goal of the dissertation was to develop realtime, direct and simple LSPR sensors to measure all types of biomolecular binding, which can be broadly classified as organic-inorganic bindings and organic-organic bindings in this study.

\subsection{Specific Aims and Hypothesis}

Specific Aim 1: Development of highly sensitive LSPR sensor and its application on the detection of small heavy metal molecule binding.

Protein conformational change induced by heavy metal molecule binding is small and undetectable using traditional surface plasmon resonance (SPR). Thus the first aim of the dissertation research was to fabricate a 3D hydrogel-nanoparticle based LSPR sensing platform for sensitivity enhancement on this binding study. ArsA ATPase and ArsD metallochaperone are two major sub-units of the arsenic detoxification pathway in charge of cytosolic arsenic extrusion and transportation, respectively. ArsA-As(III) and ArsD- 
As(III) binding was used as models for sensor evaluations.

We expected to achieve enhanced sensitivity on the detection of ArsA - As(III) and ArsD-As(III) binding with the 3D LSPR sensor comparing to the 2D LSPR sensor. The association rate constant for ArsA-As(III) and ArsD-As(III) binding under different conditions was investigated to help understanding the arsenic extrusion pathway.

Specific Aim 2: Sensing platform upgrade for biomarker study from living cell model.

Biomarkers comprise of cellular molecules such as proteins or nucleic acids that can be detected in cells, blood, urine, or other body fluids and are over-expressed due to the onset of disease. In order to measure cellular biomarkers using SPR, cells are usually lysed to extract cytosolic biomarkers. The second aim deals with the development of a direct, in situ sensing strategy to detect biomarkers released from living cells. Vascular Endothelial Growth Factor (VEGF) related to many cancers was chosen as the model analyte. It is a significant angiogenic factor that plays a crucial role in tumor growth and metastasis. This sensing platform possesses unique potential on tumor early diagnosis application, and most importantly on antineoplastic drug development.

We expected to detect VEGF from cancer cells in real-time. Some type of cancer cells have relatively low VEGF secretion rate, stimulation can be used to induce rapid exocytosis in order to measure the cell VEGF secretion level. The sensor can be used for various applications such as drug development research, cellular and tissue level cancer 
studies, etc. The successful conception of this sensor will be an important step forward in investigating VEGF and other biomarker release kinetics and drug regulation studies.

Specific Aim 3: Study of drug regulation on VEGF binding kinetics with living cell model.

Monitoring biomarker release from living cells enables the study of drug regulation on biomarkers and their receptors. Drug effects can be monitored on an in vitro living cells model which mimics the in vivo biomarker signaling pathway. In order to evaluate the LSPR sensor on drug regulation studys, a VEGF specific antineoplastic drug known as bevacizumab was used as model analytes.

We employed the sensing platform achieved in specific aim 2 to monitor the drug regulation of bevacizumab on the VEGF - VEGFR interaction. We expected to observe high association rate between bevacizumab and VEGF. Due to the neutralization effect of bevacizumab to VEGF, a lower association rate should be observed for VEGF VEGFR interaction when bevacizumab presents.

\subsection{Biosensors}

\subsubsection{Historical Survey}

Biosensors ${ }^{1}$ have received a lot of attention during the last 20 years because they find a very broad range of applications such as diagnosis and monitoring of diseases, environmental analysis, food analysis, as well as detection of illicit drugs, genotoxicity, 
chemical warfare agents, and drug screening. ${ }^{2}$ The development of biosensors incorporates well established chemical sensing techniques with a bio-recognition element. Recognition element's current impact on biosensor technology rely on entities such as antibodies, enzymes, nucleic acids, molecular imprints, or even cells and tissues. $^{3-5}$ Most biological systems have immune recognition systems through specific binding interactions. Clark et al. and others first introduced biosensors in the 1950's

combining physically entrapped enzymes to an electrochemical transducer. ${ }^{6-7}$ In recent years, research efferts on biosensors has grown exponentially with researchers from different backgrounds: engineers, chemists, physicists and clinical researchers. By the year of 2015 , the global market for biosensors is expected to grow to $\$ 12$ billion with point-of-care testing devices being the largest segment. Continuous growth in population and aging population, and the consequent increase of chronic disease is the main driving force of this market, which is expected to have continuous impact for decades. On the other hand, growing needs of biosensors for research activities within medicine industry are also innegligible. Applications such as drug discovery, drug analysis, in vivo and in vitro test of pharmacodynamic effects require numerous studies with biosensors.

\subsubsection{Definition and classification of biosensor}

Biosensors are broadly defined as analytical devices that convert a biological response, based on the composition, structure or function of a biological system, into an electrical signal. Biosensors are often used to determine the concentration of analytes or to monitor a biological system. ${ }^{6}$ 
A successful biosensor should possess the following beneficial features:

1. Sensitivity: The response of the biosensor should be accurate and precise. The signal to noise ratio of the biosensor should be high.

2. Specificity: The bio-recognition element of the biosensor must be highly specific to the analysis.

3. Stability: The biosensor must be stable within a reasonable period under normal storage conditions.

4. Reproducibility: The response of the biosensor should be reproducible and linear over a relevant analytical range, without dilution or concentration.

5. Biocompatibility: If the biosensor is to be used for in-vivo monitoring in clinical situations, the probe must be tiny and biocompatible, having no toxic or antigenic effects. If the sensor is reusable then it should be able to withstand standard sterilization procedures.

6. Economical \& labor efficiency: The complete biosensor should be cheap, small, portable and capable of being used by semi-skilled operators.

7. Market needs: There should be a market for the biosensor. There is no benefit to developing a biosensor if other factors encourage the use of traditional methods.

Biosensor generally consists of 3 parts: the sensitive biological recognition element, the signal transducer or the detector element, the biosensor reader device (Figure 1.1.). 


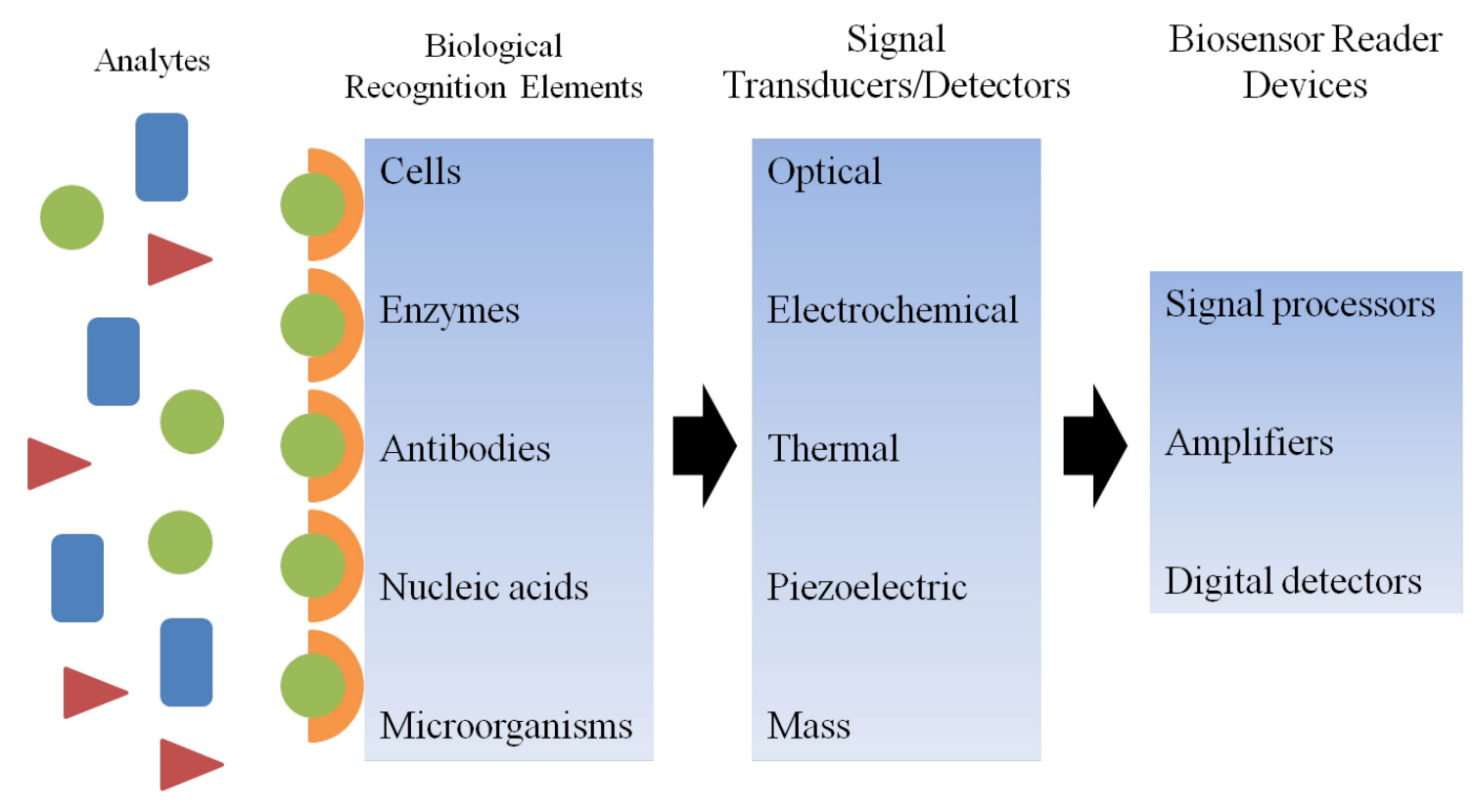

Figure 1.1. A schematic illustration of the main components of biosensors.

1. The sensitive biological recognition element, which can be, but are not limited to tissue, cells, microorganisms, cellular receptors, enzymes, antibodies, nucleic acids, aptamers, etc. Generally, it should be a biologically derived material or biomimic component that binds or recognises the analyte under study.

2. The signal transducer or the detector element (optical, electrochemical, thermal, piezoelectric, mass etc.) that transforms the signal generated by the interaction between the analyte and the biological recognition element into a signal that can be quantitivly measured.

3. The biosensor reader device consists of associated electronics (signal processors, amplifiers, digital detectors, etc.) that are responsible for the display of the data in a user-friendly manner. 


\subsection{Surface Plasmon Resonance Biosensors}

The principle of SPR biosensors is the measurement of refractive index changes at a plane interface between two media with dielectric constants of opposite signs, a dielectric and a metal such as gold (because it gives an SPR signal at convenient combinations of reflectance angle and wavelength and is chemically inert), when a wedge of polarized light, under conditions of total internal reflection, is directed towards the glass face of the sensor surface (Figure 1.2.a). ${ }^{8}$ An electric field, known as evanescent wave, is generated when the polarized light strikes the glass. This evanescent wave interacts with, and is absorbed by, free electron clouds in the metal layer, generating electron charge density waves called plasmon that propagate parallel along the metal/dielectric interface, as can be appreciated in Figure 1.2.b by Badia et al., causing a reduction in the intensity of the reflected light. No photons exit the reflecting surface, but their electric field decreases exponentially with distance from the interface, decaying over a distance of $\sim 1 / 4$ wavelength beyond the surface. The resonant angle at which a minimal in intensity of reflected light occurs is a function of the local refractive index at or near the gold surface. ${ }^{9-11}$ Thus, the most important point in relation to the experiments is that a change in the bulk refractive index of the dielectricum medium and the adsorption or desorption of molecules from the metal surface changes the refractive index at the metal-dielectric interface and results in changes in the SPR signal. LSPR is excited by a similar method to SPR excitation. However, SPR is generated on a planar metal surface, whereas LSPR is generated on a nano-structure surface. Such nano-structure causes a shorter decay length of light when penetrating the surface, therefore offers a higher spatial resolution and higher sensitivity than SPR. 

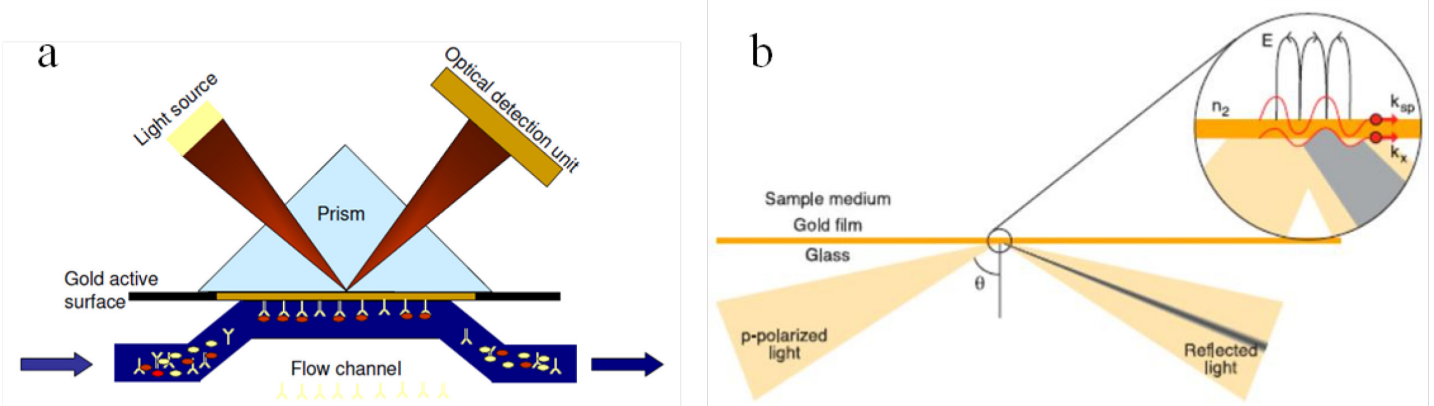

Figure 1.2. (a) Kretschmann configuration SPR, (b) Plasmon propagation parallel along the metal/dielectric interface

SPR is generated by linearly polarized light, with its electric (E) field vector elther parallel or perpendicular (Figure 1.3) to the plane of incidence. Such polarized light is named p- or s- polarized light. For the p-polarized light illustrated in Figure 1.3, there is an E-field component perpendicular to the interface $E_{z}=|E| \cos (\theta)$. As the definition of dielectric permittivity, $\varepsilon_{1}=\left(\mathrm{n}_{1}\right)^{2}$ and $\varepsilon_{2}=\left(\mathrm{n}_{2}\right)^{2}$ (medium $1=$ glass, medium $2=$ metal). We assume that the media does not absorb light: $\varepsilon_{0} \varepsilon_{1} \mathrm{E}_{\mathrm{z} 1}=\varepsilon_{0} \varepsilon_{2} \mathrm{E}_{\mathrm{z} 2}$.

Since $\varepsilon_{1} \neq \varepsilon_{2} \quad\left(\mathrm{n}_{1} \neq \mathrm{n}_{1}\right) \rightarrow \mathrm{E}_{\mathrm{z} 1} \neq \mathrm{E}_{\mathrm{z} 2}$, the change in $\mathrm{E}_{\mathrm{z}}$ means the polarization charge distribution across the interface is discontinuous. This change in $\mathrm{E}_{\mathrm{z}}$ will give rise to the creation of electron charge density waves at the boundary. On the other hand, s-polarized light has no $E_{z}$ component, it cannot be utilized for generation of SPR. 


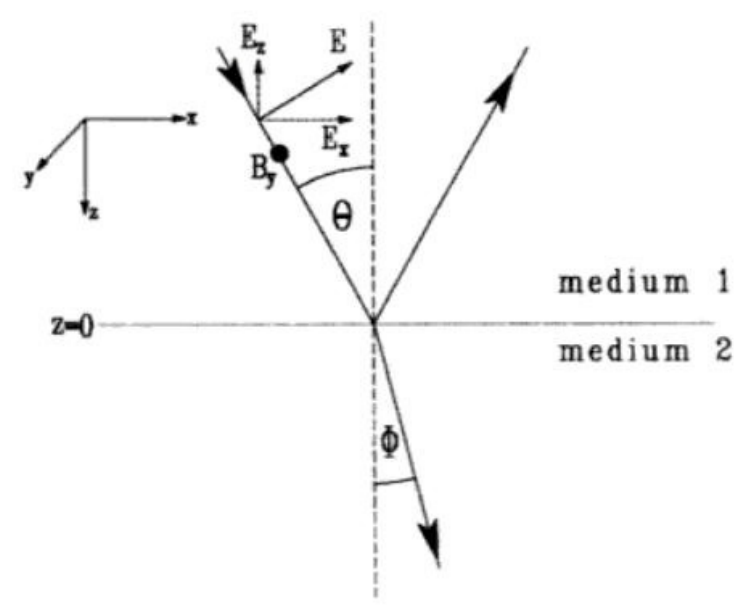

Figure 1.3. Illustration of p-polarized light made incident on a planar interface of two medium. For a typical SPR instrument, medium 1 is a dielectric material such as glass, medium 2 is a metal such as gold.

Earlier applications of LSPR biosensors were mainly focused on the interaction characterization of single molecules between each other: protein/protein, nucleic acid/protein, or nucleic acid/nucleic acid interactions. ${ }^{12-13}$ In 2008, Miura's group demonstrated a prototype field portable LSPR system using monoclonal trinitrotoluene (TNT) antibody modified AuNPs for detection of TNT. ${ }^{14}$ They achieved a detectable range of $10 \mathrm{ppt}$ to $100 \mathrm{ppb}$, which is four-fold more sensitive than that in the absence of AuNPs. Using a similar principle, Kim's group recently constructed an LSPR biosensor based on subwavelength 1D and 2D gold nanoarrays built on a thin gold film for the detection of avian influenza DNA hybridization. ${ }^{15}$ Their results showed that 1D nanogratings exhibited four-fold amplification of the SPR signal, and 2D nanohole arrays exhibited a 2.5-fold increase in amplification. Although such technologies have successfully demonstrated high detection sensitivity in the range of picomolar of analytes, small molecules such as heavy metal ions have had difficulty being measured 
using this current setup. On the other hand, studies have addressed cellular structures, ${ }^{16}$ such as artificial membranes ${ }^{17}$ and even whole biological organisms ${ }^{18}$ as interaction partners play a pivotal role. Especially for the development of medical and pharmaceutical assay formats, utilizing LSPR biosensors, the shift from single-molecules interactions to the whole cell system with its complex regulation pathways is necessary. Many cellular activities can be evaluated by the change of cell secretions. SPR has also been utilized on cellular level studies through detecting proteins or biomarkers in cell secretions. In 2007, Li et al. demonstrated the detection of VEGF using SPR with detection sensitivity down to subpicomolar. RNA aptamer microarrays were used to capture the VEGF on the sensing surface. Another antibody-horseradish peroxidase layer was linked on top of the VEGF as a sandwitch layer. 5, 5'-tetramethylbenzidine was also immobilized on the sensing surface. The localized horseradish peroxidase - 5,5'tetramethylbenzidine precipitation reaction was used to amplify the SPR response. ${ }^{19} \mathrm{Yu}$ et al. studied the interaction between Bevacizumab (an antibody based drug for VEGF regulation) and VEGF using SPR in 2008. Bevacizumab was found to specifically bind to human VEGF. It is not a suitable drug for studies involving murine VEGF. ${ }^{20}$ Similar to these two studies, many published studies on the detection of proteins and biomarkers exocytosis using SPR only demonstrated the detection of analytes from solution. Moreover, Kyo et al. developed a SPR sensing platform to detect proteins from crude cells lysate. This study has shown the specificity of SPR with a real biological fluid. ${ }^{21}$ Another research group developed a SPR based sensing strategy to monitor the intracellular superoxide dismutase in $E$ coli.. ${ }^{22}$ Both of these stuies have achieved sensitive, label-free detection of analytes from cells. However, cells were lysed to collect 
analytes for SPR measurements. The lysing process may potentially induce variations to cellular secretions and cause false results. Till date, there is a lack of a non-invasive SPR sensing platform for cellular secretion studies.

Figure 1.4 shows the BI-2000 flow injection SPR instrument that we employed in this dissertation study. The instrument consists of two modules: a control module and a precision syringe pump. The control module generates a $670 \mathrm{~nm}$ laser for the excitation of SPR. This laser makes incident on the SPR sensing surface after going through a prism. A flow cell is attached on top of the sensing surface. The precision syringe pump and the sample injection port are connected to the flow cell to provide a continuous flow of environmental buffer and to introduce samples to the sensing surface, respectively.

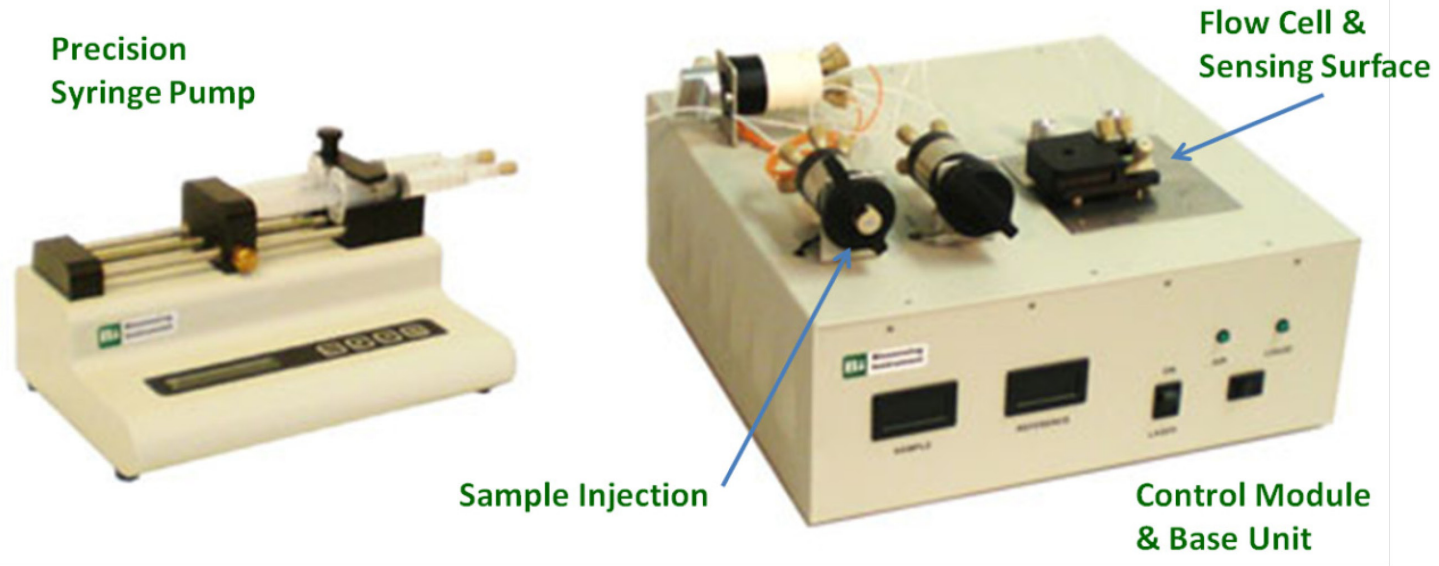

Figure 1.4. A picture of the BI-2000 flow injection SPR instrument.

A typical SPR sensorgram usually consists of the following curves (Figure 1.5):

1. Baseline: A stable baseline is the reference for SPR angle shift induced by surface binding. It is usually obtained by washing the surface with flowing buffer for 3$5 \mathrm{~min}$. 
2. Association curve: After the injection of the analyte, SPR basline will increase and reach a maximum point. This process indicates the analyte passing through the surface and binding to the surface ligand. Binding affinity information can be obtained from this curve.

3. Dissociation curve: This process occur at the end of the injection, unbound analyte is carried away by the flowing buffer. The baseline shift value (mDeg) after the dissociation curve bears information of the concentration of analyte.

4. Regeneration (optional): The sensing surface can be regenerated by reagent such as $\mathrm{HCl}$ or $\mathrm{NaOH}$ for reuse.

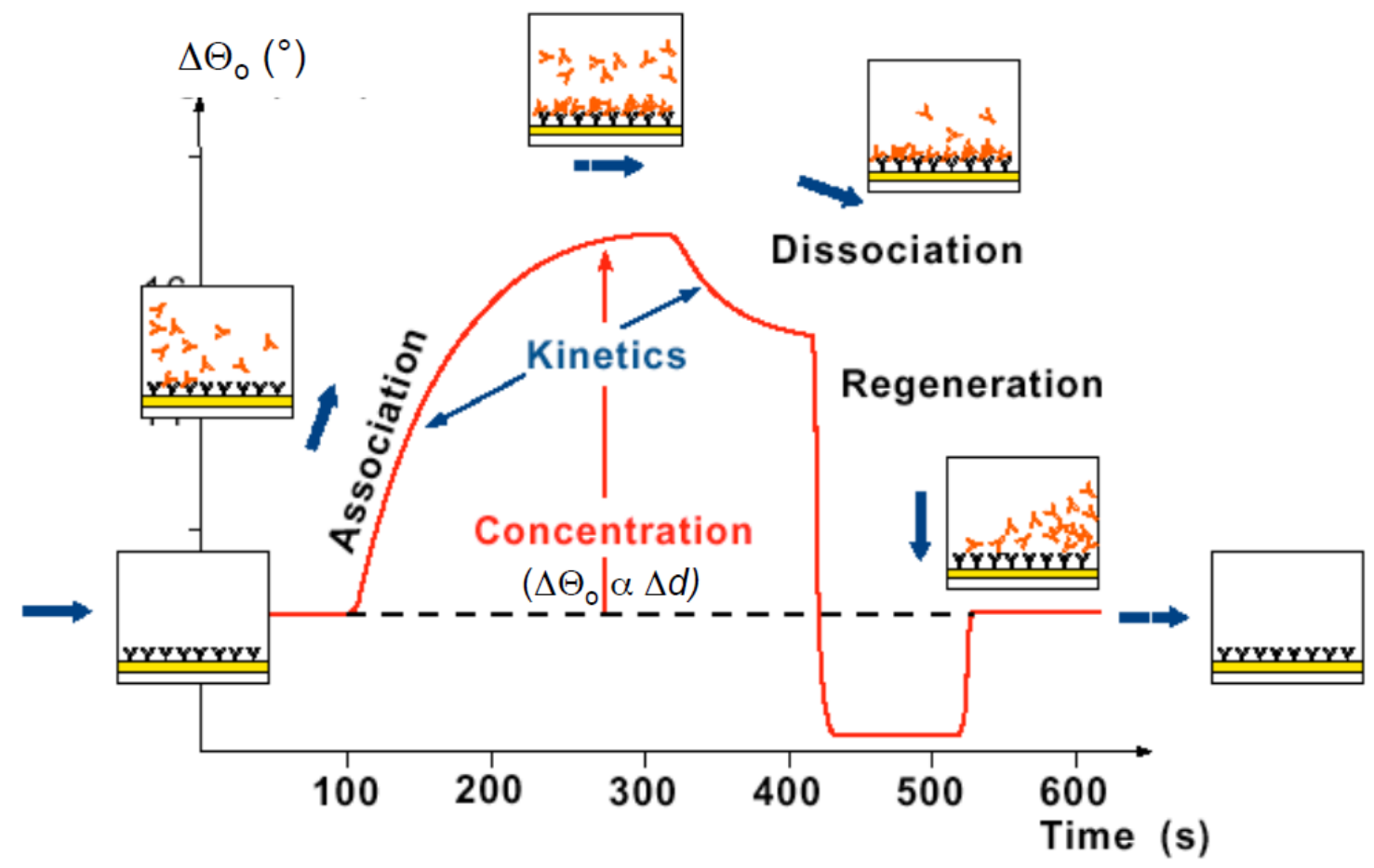

Figure 1.5. The sensorgram of baseline, association, dissociation and regeneration processes during a typical SPR experiment. (laboratory for genomics and bioinformatics, University of Oklahoma health sciences center)

Furthermore, recent developments of LSPR sensors focusing on a reduction in device 
size, ${ }^{23}$ imaging applications, ${ }^{24}$ and multichannel sensing ${ }^{12}$ have increased their significance. This great interest and development of LSPR sensors is due to its high sensitivity, selectivity, speed, and reliability in analysis.

\subsection{Other Methods for Binding Measurements}

\subsubsection{Current Methods to Measure Protein-Arsenic binding}

Current methods used for As (III)-Ars proteins binding study are extended x-ray absorption fine structure (EXAFS), fluorescence excitation spectra, gel filtration, etc. ${ }^{25}$ However, all aforementioned methods are associated with several disadvantages and limitations. EXAFS is a definitive method for analyzing metal ligands but lacks sensitivity and real-time analysis. It thus requires intensive labor to reveal binding kinetic information from uncontinuous data. Furthermore, EXAFS technique necessitates highly sophisticated, expensive detection equipment. Fluorescence excitation spectra provide real time data acquisition in the millisecond range but cannot offer direct binding information. Binding process is studied by measuring changes in fluorescence anisotropy. Gel filtration technique for protein separation lacks accuracy due to residue on columns. In addition, most of these equipments require trained personnel to use and maintain which add to the diagnosis costs. Therefore, these techniques are not suitable to be used to study real-time binding affinity and kinetics.

\subsubsection{Current Methods to Measure Biomarkers}

Enzyme-linked immunosorbent assay (ELISA), fluorescence correlation spectroscopy, electrochemistry and Western Blotting, etc. are commonly used methods for detection of 
biomarkers. $^{26-27}$ However, all aforementioned methods are associated with several disadvantages and limitations. For instance, ELISA technique lacks sensitivity, reproducibility and real-time dynamic detection capabilities. Immunoassay techniques also require intensive labor work from trained personnel. Fluorescence spectroscopy techniques may be used for highly sensitive biomarker detection, but they require labeling samples with fluorescence dye. Electrochemistry sensing techniques offer time and cost effective, highly sensitive strategy for biomarker detection. However, the major limitation of electrochemistry sensing is that it requires sample to be electrochemically active. SPR sensing platform in this proposed work is widely used in the past two decades in the application of diagnosis and monitoring diseases, environmental analysis, food analysis, as well as drug screening. SPR constitutes a very interesting alternative due to their advantages in the study of biomarker: ${ }^{28}$

- High sensitivity (subnanogram mass or subnanometer thickness of the surface bound layer)

- No need to label the analyte molecules, minimizing the potential negative impact of the label on their biological or chemical function.

- Real-time monitoring of recognition processes in situ; this constitutes an important driving force in biosensor research focused in the development of largescale biosensor arrays composed of highly miniaturized signal transducer elements that enable the real-time and parallel monitoring of multiple species.

- Ability to detect interactions at solid/liquid interfaces, which are involved in many biologically relevant recognition processes.

However, there are also several disadvantages associated to the SPR biosensor. The main 
disadvantages are their high cost and lack of portability. A typical commercialized SPR instrument can cost $\$ 50,000$ to 500,000 with a size larger than a desktop computer. SPR is also lack of simultaneous measurement capability, which makes it not suitable for high throughput applications.

\subsection{Polymer Brushes for Biosensing Signal Enhancement}

Polymer brushes may form ultrathin coatings, usually from block copolymers or endgrafted polymers that are tethered with one chain end to an interface, which is generally a solid substrate. ${ }^{29}$ Polymer brushes exhibit a behavior that is comparable with selfassembled monolayer (SAM). If appropriate monomers are used, they provide a pathway for the fabrication of versatile adaptive surfaces capable of responding to changes of temperature, solvent polarity, $\mathrm{pH}$, and other stimuli, generally by reversible swelling. ${ }^{30}$ Polymer brushes are mainly applied onto surfaces by: (a) Physisorption; (b) LangmuirBlodgett technique; (c) The "grafting to" approach, also called, chemisorption, which involves the formation of covalent bonds between a polymer previously prepared and the surface; and (d) The "grafting from" approach, which involves the polymerization of the monomer or monomers from functionalized surfaces (also called surface initiated polymerization, SIP, or surface mediated polymerizations, SMP). This work employed surface initiated radical polymerization for the following significant advantage:

- Better control of brush thickness, composition, and architecture

- Broad choice of monomer

- Compatibility with both aqueous and organic media

- High tolerance toward a wide range of functional groups 
Poly(N-isopropylacrylamide) (PNIPAAm) is one of the most widely studied polymer. ${ }^{31-32}$ This non-ionic polymer undergoes a sharp hydrophilic-hydrophobic transition in aqueous solution at $32^{\circ} \mathrm{C}$. Such property that has a variety of applications in many fields, including solute extraction and separation, controlled drug release, ${ }^{33}$ artificial organs, ${ }^{34}$ and enzyme immobilization, with the swellable hydrogels prepared by polymer crosslinking. PNIPAAm brushes have previously been grafted form different substrates and employed for the fabrication of functional surfaces capable of a reversible transition between hydrophilic and hydrophobic character when subjected to changes in temperature, and also have been combined with nanopatterning strategies. Other polymers such as poly(acrylic acid) (PAAc) and poly(vinyl pyridine) (PVP) respond to changes in $\mathrm{pH}^{35}$ PAAc, which is hydrophobic in its protonated state $(\mathrm{pH}<4)$ becomes hydrophilic after deprotonation. ${ }^{36}$ PVP is also a $\mathrm{pH}$ dependent polymer that behaves as a hydrogel ${ }^{37}$ it is hydrophobic in its deprotonated state $(\mathrm{pH}>5)$ and becomes water soluble in its protonated state $(\mathrm{pH}<5)$. In spite of the characteristics of the intelligent polymers previously mentioned, the preparation of their copolymers results more interesting for sensing applications, since the combination of polymers with different characteristics allow the modulation of the final properties of the materials in the process that couples molecular recognition with swelling and shrinking processes.

\subsection{Arsenic Detoxification Research}

Arsenic is one of the most prevalent environmental toxins. The natural content of arsenic found in soils varies between $0.01 \mathrm{mg} / \mathrm{kg}$ and a few hundred milligrams per kilogram. Arsenic is a component of over 245 minerals. Volcano activities and other geological 
process are sources of the high arsenic concentrations in the environment. ${ }^{38}$ Human activities are also significant sources of arsenic contamination in water and soil:

- The disposal of industrial waste chemicals

- The smelting of arsenic bearing minerals

- The burning of fossil fuels

- The application of arsenic compounds in many products especially in the past few hundred years

Arsenic exists in nature in the oxidation states $+\mathrm{V}$ (arsenate), $+\mathrm{III}$ (arsenite), 0 (arsenic) and -III (arsine). In the aqueous environment, inorganic arsenic commonly exists in oxidation states as arsenous acid (As(III)), arsenic acid $(\operatorname{As}(\mathrm{V}))$, and their salts. Organic arsenic compounds such as monomethylarsonic acid (MMA), dimethylarsinic acid (DMA) are usually less toxic than inorganic arsenic compounds. Acute and chronic arsenic poisoning may cause disorder of cardio-vascular, nervous, respiratory and gastrointestinal system. Moreover, Arsenic is a carcinogenic and may cause lung cancer, bladder cancer, liver cancer and etc.

Today, arsenic contamination in drinking water is commonly found in India (WestBengal), Vietnam, Taiwan, Mexico, Argentina, Chile, Hungary, Romania, and many parts of the United States. ${ }^{39-40}$ WHO research reveals that over 40 million people are acute at risk of arsenic groundwater problems in Bangladesh. Consequently, the chronic 
toxicity of arsenic will be a common cause of death in Bangladesh.

\subsection{Current Chemotherapeutic Therapy and VEGF Regulation Therapy}

Chemotherapy is the treatment of cancer with an antineoplastic drug or with a combination of such drugs. It is the most commonly used treatment for a vast majority of human tumors. Principally, chemotherapeutic drugs kill cells with a rapid dividing rate. However, they also harm cells that divide rapidly under normal circumstances, such as cells in the bone marrow, digestive tract and hair follicles, thus results in the most common side effects of chemotherapy: myelosuppression, mucositis and alopecia. ${ }^{41}$ In the broad sense, most chemotherapeutic drugs work by impairing mitosis, effectively targeting fast-dividing cells and cause cells to undergo apoptosis.

The majority of chemotherapeutic drugs can be divided into following categories: antimetabolites, alkaloids and terpenoids inhibitors, cytotoxic antibiotics and other antitumour agents. Doxorubicin is one of the most widely studied cytotoxic antibiotics. It is commonly used to treat a wide range of cancer but with a serious adverse effect of heart damage. Doxorubicin interacts with the cell DNA by intercalation and inhibition of molecular biosynthesis, preventing the DNA double helix from being resealed and thereby stopping the process of replication. ${ }^{42}$ Bevacizumab is a humanized monoclonal antibody that inhibits VEGF-A. It is currently used as an angiogenesis inhibitor to stop tumor growth by preventing the formation of new blood vessels. Thrombospondin (TSP) is an antiangiogenic which inhibits the proliferation and migration of endothelial cells by interactions with CD36 expressed on their surface of these cells. 
All aforementioned antineoplastic solutions are controversial due to adverse effects, molecular weight, unclear mechanism and limited availability. Therefore, research for development of newer anticancer drugs one of the most funded research by National Institutes of Health (NIH). During the period from 2005 through 2010, the National Cancer Institute $(\mathrm{NCI})$ budget averaged $\$ 4.9$ billion per year.

Human cells rely on a constant oxygen supply to maintain their cellular activity. Therefore all of our cells are within a tenth of a millimeter from a blood capillary which delivers oxygen via blood to cells. Tumor cells are no exception and even more hungry on blood supply. A tumor larger than a millimeter will starve itself of oxygen and energy unless new blood vessels are built to provide a supply. For this reason, many cancer cells employ the normal processes of angiogenesis in order to build their own blood supply. ${ }^{43}$

VEGF is a signal protein produced by oxygen-hungry cells to promote growth of blood vessels. It binds to specialized receptors on the surfaces of endothelial cells and directs them to build new vessels. Tumor cells use the same process to build their own oxygen supply. They produce abnormally large amount of VEGF or block the action of angiogenesis inhibitors. This action is termed as "angiogenic switch", giving the ability of metastasis to the tumor, since a custom-made blood supply can be constructed wherever new tumors begin to grow. ${ }^{44-45}$

VEGF secretion level directly reveals the tumor size, growth stage and malignancy. 
Therefore, VEGF secretion rate measurement from living cancer cell culture is essential to prediction and early diagnosis of cancers. Taking advantage of the highly sensitive detection capabilities of SPR, we used it to measure VEGF secretion amount from a certain number of cancer cells in real-time, thus to calculate the average single cell VEGF secretion rate. The time resolution of VEGF release was monitored previously on a scale of several hours or days due to the limitations of the detection techniques available. By using SPR biosensors we were able to measure VEGF release on a scale of microseconds. Due to the highly sensitive detection possible using SPR even the slightest release of VEGF from the cancer cells can be instantaneously mapped.

VEGF secretion is also an attractive process for the development of newer cancer therapy. Blood supply to the tumor can be cut off by selective inhibition of VEGF production or binding to its receptor using drugs or antibodies. ${ }^{46}$ A large amount of drugs and antibodies are currently being tested by different research groups to modulate VEGF production in cancer cells. To develop suitable drugs for this propose, bind process of VEGF-drug or VEGFR-drug interaction is a significant factor. The unique real-time recoding feature of SPR enables us to monitor the association and dissociation curve of the binding process, which provided the binding association constant value for each drug sample. 


\section{Chapter 2}

Development of the highly sensitive LSPR sensor and its application on the detection of small heavy metal molecule binding 


\subsection{Introduction}

Surface plasmon resonance has brought a revolutionary change to in vitro study of biological and biochemical processes due to its ability to measure extremely small changes in RI, binding equilibrium and kinetics. ${ }^{47-51}$ Strategies based on plasmonic nanoparticles have been employed to enhance the sensitivity for a variety of applications, such as diagnosis of diseases, environmental analysis, food safety, and chemical threat detection. $^{52}$ Theoretically, SPR can be induced only in free electron metals, e.g., Au, Ag and $\mathrm{Cu}$, due to the interaction of surface electrons with the electromagnetic wave and the contribution from the interband transition of the d-shell electrons. ${ }^{53}$ At the end of the 1990s, several research groups had begun exploring schemes for the development of LSPR biosensors using noble metal nanoparticles because of the extremely sensitive nature of their electron-rich surfaces to the surrounding environment. Based on the Mie theory, when an electromagnetic wave is directed to the metallic nanoparticle, an induced oscillation of free electrons occurs at the surface, resulting in a characteristic extinction spectrum that depends on the type of metal, the size and shape of the nanoparticles,

interparticle distance and most importantly, the RI of the surrounding medium. ${ }^{54-55}$ Compositional and conformational changes within the surrounding dielectric medium near a nanoparticle could therefore be detected as shifts in the extinction spectrum. LSPR spectroscopy operates in a manner that is analogous to SPR to induce the extinction spectrum, however, the electromagnetic field of LSPR decays within a much smaller length than in SPR, which gives significant rise to the sensitivity of LSPR sensors. 
The fabrication of the metallic nanostructure on the LSPR sensing layer is traditionally performed by "2D" methods. DebRoy's group immobilized polyclonal antibodies for Escherichia coli (E.coli) O157:H7 using biotin-neutravidin binding to detect E. coli O157:H7 spiked in pasteurized milk (skim-milk), apple juice, and ground beef extract. ${ }^{56}$ They also functionalized a SPR gold chip with carboxymethylated dextran layer followed by Protein A to immobilize polyclonal antibodies against E. coli or Salmonella Enteritidis. ${ }^{57}$ In these cases, low concentration targets in the sample were difficult to detect because of the limited interaction time with the sensing surface due to continuous flow. Signal amplification is a common strategy for detection of low concentrations of target molecules. Cheng's research group recently reported a novel SPR signal amplification strategy based on in situ surface-initiated atom transfer radical polymerization, ${ }^{58}$ in which a polymer was used as a label for small molecules. Another feasible strategy is the functionalization of the SPR sensor chip with an absorbtive coating in addition to using bare nanoscale noble metal structures for amplifying the sensor response. Radical copolymers would be ideal materials for this purpose due to their high capacity for absorbing the analyte via a swelling-shrinking process upon interacting with a water based buffer, enabling the sensing surface to capture larger amount of analyte. ${ }^{59}$ This property allows interparticle distance tuning. Poly (Nisopropylacrylamide) is a promising material that can satisfy these requirements. ${ }^{60}$ Furthermore, copolymers obtained from the combination of polymers may result in even more sensing applications, as polymers with different functional groups allow the modulation of the material's final properties for recognizing different analytes. Finally, radical copolymers prevent non-specific binding to the remaining free gold surface in 
between the probes. This method is comparable to the conventional immunoassay approach in which the surface is masked with a high concentration of a non-specific protein such as bovine serum albumin or denatured casein. ${ }^{61}$

Arsenic (As) is one of the most common toxic elements in the environment and is introduced by both geochemical and anthropogenic sources. Exposure to this metalloid leads to cancer, cardiovascular and peripheral vascular diseases, diabetes and neurological disease. ${ }^{62}$ The field of arsenic detoxification has been studied for many decades. Nearly every organism, from bacteria to humans, has an arsenic detoxifying system. In bacteria and archaea, the genes for arsenic resistance are usually found in arsenical resistance (ars) operons. One such operon, the ars operon of plasmid R773, produces resistance to trivalent and pentavalent salts of the metalloids arsenic and antimony in cells of E. coli catalyzed by an Adenosine-5'-triphosphate (ATP)-coupled $\mathrm{As}(\mathrm{OH})_{3}$ extrusion pump. ${ }^{63}$ The operon has five genes, arsRDABC. ${ }^{64}$ Among them, ArsR is a transacting repressor protein that homeostatically regulates the levels of ars transcription. ${ }^{65}$ ArsD is an As(III) chaperone that binds and transfers cytosolic arsenite to ArsA, an As(III)-activated ATPase. ${ }^{66}$ Together ArsA and ArsB, a transmembrane arsenite antiporter, form the ATP-driven $\operatorname{ArsAB} \mathrm{As}(\mathrm{OH})_{3}$ extrusion pump. ${ }^{67}$ In 2006, Tao's group reported the first application of SPR sensing for arsenic detection in groundwater. ${ }^{68}$ However, there are no reports that utilize this technique for kinetics study on arsenic transportation and detoxification to date.

In this study, we integrated in situ radical copolymerization and AuNPs to construct a 
novel LSPR sensing system. By growing 3D AuNPs-doped PNIPAAm-co-PAAc hydrogel-based coating on the gold sensing surface, we observed a 6.8-fold enhancement of LSPR signal comparing to the traditional 2D AuNPs-decorated gold sensing surface. We applied this LSPR sensor to study the binding kinetics of the ArsA ATPase for As(III) in the presence of adenosine-5' -triphosphate and magnesium ion (MgATP) and ArsD. In previous studies, binding of As(III) by Ars proteins was performed by methods such as EXAFS. ${ }^{25}$ EXAFS is a definitive method for analyzing metal ligands but lacks sensitivity and real-time analysis. In contrast, our 3D hydrogel based LSPR sensing strategy obtained direct, real-time binding kinetic information. ${ }^{69}$ Thus, in addition to providing a means of amplifying the LSPR response, this work presents a novel approach to study the kinetic behavior of the arsenic extrusion pathway. Finally, the functionality of our novel 3D hydrogel-nanoparticle coating can be easily modified by changing or adjusting loading of monomers, it has potential to be broadly applied to sensing a range of biological analytes.

\subsection{Experimental}

\subsubsection{Materials}

Allylmercaptan, acrylic acid (AAc), N-isopropylacrylamide (NIPAAm), N,Nmethylenebisacrylamide (BIS) were purchased from Acros Organic (New Jersey), 2,2'azobisisobutyronitrile (AIBN) and diisopropylfluorophosphate (DIFP) were obtained from Sigma-Aldrich (St. Louis, MO). 11- mercaptoundecanoicacid (MUA) was purchased from Asemblon (Redmond, WA). Cystamine dihydrochloride was obtained from Spectrum (New Brunswick, NJ). 1-ethyl-3-(3-dimethylaminopropyl) carbodiimide 
hydrochloride (EDC), N-Hydroxysuccinamide (NHS) and hydrogen tetrachloroaurate $\left(\mathrm{HAuCl}_{4}\right)$ were obtained from Alfa Aesar (WardHill, MA). Trisodium citrate $\left(\mathrm{Na}_{3} \mathrm{C}_{6} \mathrm{H}_{5} \mathrm{O}_{7}\right)$ and dimethyl sulfoxide (DMSO) were purchased from Fisher Scientific (Waltham, MA). All reagents and solvents were used as received.

\subsubsection{Protein expression and purification}

Cells bearing the indicated plasmids were grown in Lysogeny Broth (LB) medium over night at $37^{\circ} \mathrm{C}$ and then diluted 50 -fold into $1 \mathrm{~L}$ of the same medium. Proteins were expressed by induction with $0.3 \mathrm{mM}$ isopropyl- $\beta$-D-thiogalactopyranoside at $\mathrm{A}_{600}$ of $0.6-$ 0.8 for $3 \mathrm{~h}$. ArsA with a six histidine tag at the C-terminus was purified from cells of strain BL21 (DE3) expressing pAlter-1-dAhB plasmid, as described. ${ }^{70}$ Cells were harvested by centrifugation and washed once with a buffer containing $50 \mathrm{mM} 3-(\mathrm{N}-$ morpholino)propanesulfonic acid (MOPS), $\mathrm{pH} 7.5,0.5 \mathrm{M} \mathrm{NaCl}, 30 \mathrm{mM}$ imidazole and 10mM 2-mercaptoethanol (Buffer A). The cells were suspended in $5 \mathrm{~mL}$ of Buffer A per gram of wet cells and lysed by a single passage through a French press at 20,000 psi. DIFP was added at $2.5 \mathrm{~mL} / \mathrm{g}$ wet cells immediately following French press. Unbroken cells and membranes were removed by centrifugation at $150,000 \mathrm{xg}$ for $1 \mathrm{~h}$ at $4^{\circ} \mathrm{C}$. The supernatant was loaded to $10 \mathrm{~mL}$ Probond Ni-column (Invitrogen) pre-equilibrated with Buffer A. Unbound proteins were washed by $60 \mathrm{~mL}$ of buffer A, and ArsA was eluted with imidazole gradient generated by Buffer A and Buffer B (50 mM MOPS, pH 7.5, $0.5 \mathrm{M} \mathrm{NaCl}, 300 \mathrm{mM}$ imidazole and 10mM 2-mercaptoethanol), followed by addition of $0.25 \mathrm{mM}$ Ethylenediaminetetraacetic acid (EDTA) and $5 \mathrm{mM}$ Dithiothreitol (DTT) to each fraction. ArsA containing fractions were identified by sodium dodecyl sulfate 
polyacrylamide gel electrophoresis (SDS- PAGE), pooled, concentrated by Amicon Ultra-15 Centrifugal Filter Unit with Ultracel-10 membrane (Millipore), mixed with 10\% glycerol, aliquoted and stored at $-80^{\circ} \mathrm{C}$ until use. ArsD and its derivatives with a six histidine tag at the N-terminus were purified similarly. Purified proteins were stored at $80^{\circ} \mathrm{C}$ until use, and their concentrations were determined according to the method of Bradford $^{71}$ or from the absorption at $280 \mathrm{~nm}^{72}$

\subsubsection{Preparation of gold nanoparticles}

Colloidal gold nanoparticles used in this study were prepared by citrate reduction of $\mathrm{HAuCl}_{4}$ in aqueous solution. ${ }^{73}$ The formation of gold nanoparticles can be observed by a change of color. Briefly, $\mathrm{HAuCl}_{4}$ and sodium citrate solutions were filtered through a $22 \mu \mathrm{m}$ microporous membrane filter prior to use. $\mathrm{HAuCl}_{4}(40 \mathrm{~mL}, 1.0 \mathrm{mM})$ was then added to an Erlenmeyer flask (250mL), vigorously stirred and brought to a boil on a hot plate. Following this, $3.5 \mathrm{~mL}$ of $1 \%$ trisodium citrate was added to the vortex of the boiling solution. $100 \mathrm{~nm}$ Au particles were formed $2 \mathrm{~min}$ after the addition of trisodium citrate and $15 \mathrm{~nm}$ AuNPs were obtained if the solution was stirred for an additional 10 min. A layer of absorbed citrate anions on the surface of the nanoparticles prevents aggregation. The particle size was determined by a ZEN3600 Zetasizer from Malvern Instruments, Inc. (Westborough, MA).

\subsubsection{In situ polymerization}

A stock of amino group modified AuNPs was prepared by incubating bare AuNPs in $1 \mathrm{mM}$ cystamine dihydrochloride solution for $12 \mathrm{~h}$ at $4^{\circ} \mathrm{C}$. Following this, $400 \mu \mathrm{mol}$ Aac 
was added to the amino modified AuNPs and incubated under the presence of NHS/EDC for 30min to form covalent linkage $(\mathrm{pH}=7.4)$. The resulting solution was then centrifuged at $7000 \mathrm{RCF}$ for $10 \mathrm{~min}$ and the sediment was washed and resuspended with a same volume of DMSO for further use (not shown in Scheme 2.1.). A gold SPR chip was cleaned using piranha solution for 2 min and rinsed with copious ethanol. The cleaned gold chip was incubated in a $10 \mathrm{mM}$ allylmercaptan/ethanol solution afterward for $12 \mathrm{~h}$ at room temperature in the absence of light. Lastly, the chip was rinsed three times with ethanol and DMSO shortly prior to use to remove all the unbounded allylmercaptan residual. The in situ polymerization was carried out in the following manner: $5 \mathrm{~mL}$ DMSO containing $950 \mu \mathrm{mol}$ of NIPAAm, $150 \mu \mathrm{mol}$ of AuNPs linked AAc, $250 \mu \mathrm{mol}$ of AAc, $58 \mu \mathrm{mol}$ of cross linking agent BIS and $805 \mu \mathrm{mol}$ of AIBN as the initiator were first added along with the gold chip into a three-neck round bottom flask (Figure 2.1). The resulting solution was degassed by passage of a stream of nitrogen for a minimum of $20 \mathrm{~min}$ and then heated at $60^{\circ} \mathrm{C}$ for $110 \mathrm{~min}$ in nitrogen environment (Scheme 2.1). After polymerization, the AuNPs doped PNIPAAm-co-PAAc polymercoated gold chip was 
A

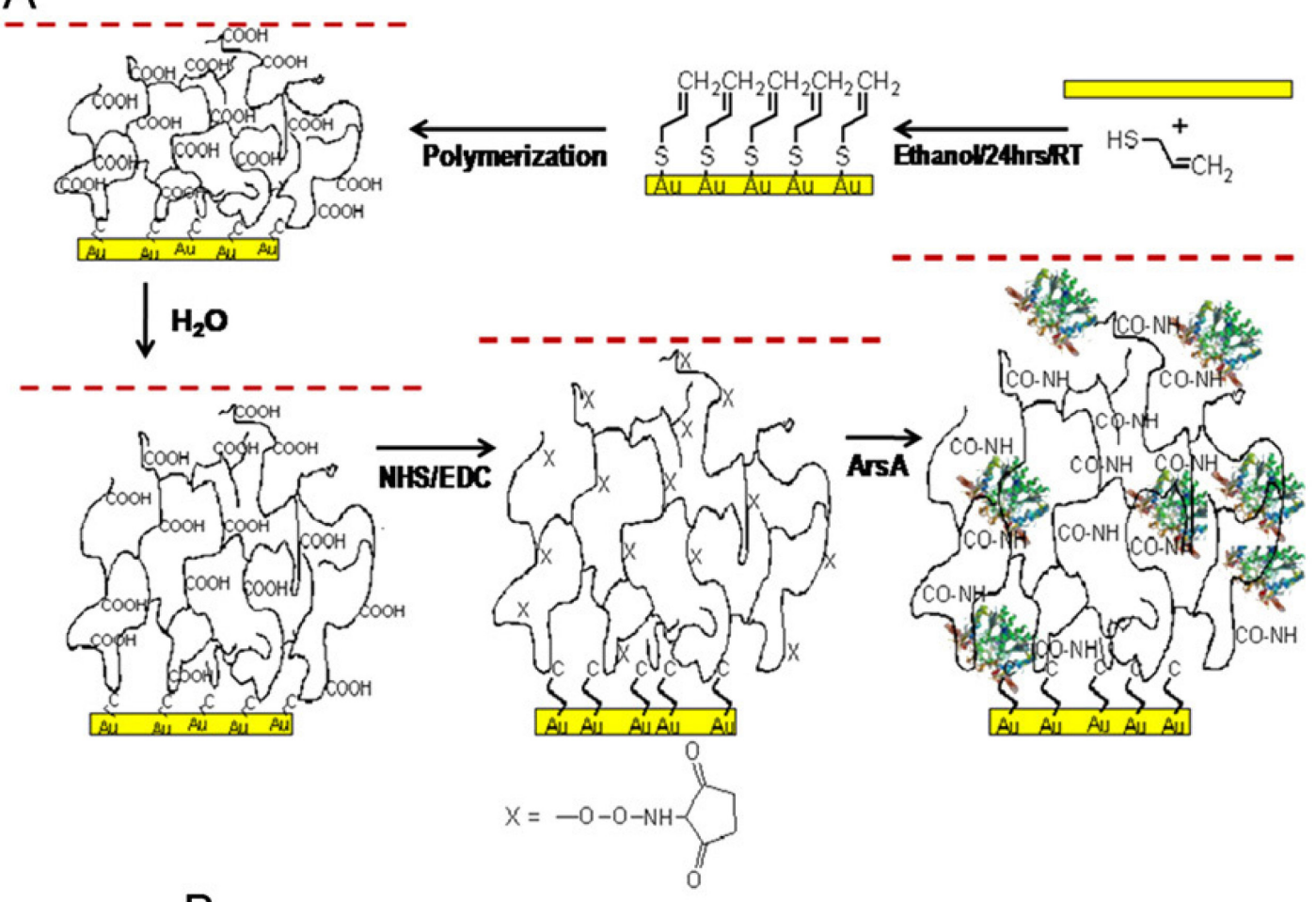

B

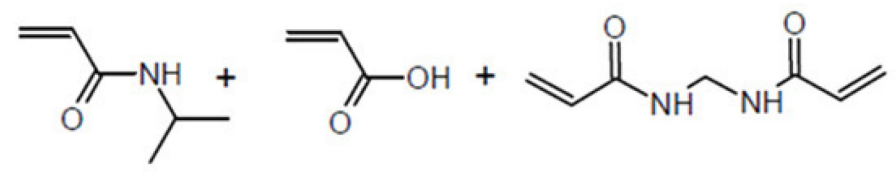

Nisopropylacrylamide Acrylic Acid M,Nmethylenebisacrylamide NIPAAm

AA

BIS<smiles>[R]CCC(CC(CC(CC(CC(CC(C[R2])C(=O)O)C(=O)NC(C)C)C(=O)NC(C)C)C(=O)NC(C)C)C(=O)NC(C)C)C(=O)NC(C)C</smiles>

Scheme 2.1. (A) Cartoon representation of the swelling-shrinking process of the copolymer for LSPR signal amplification and immobilization of ArsA ATPase. (B) Structural illustration of the in situ radical copolymerization of PNIPAAm-co-PAAc hydrogel. 
washed with DMSO, ethanol and water in order to remove any non-bonded copolymer and unreacted monomers. Finally, the chip was stored under vacuum to remove any water absorbed in the hydrogel before LSPR experiment. Control chips were also prepared by deposition of bare AuNPs on SPR chips and functionalized with carboxylic groups using MUA for characterization purposes.

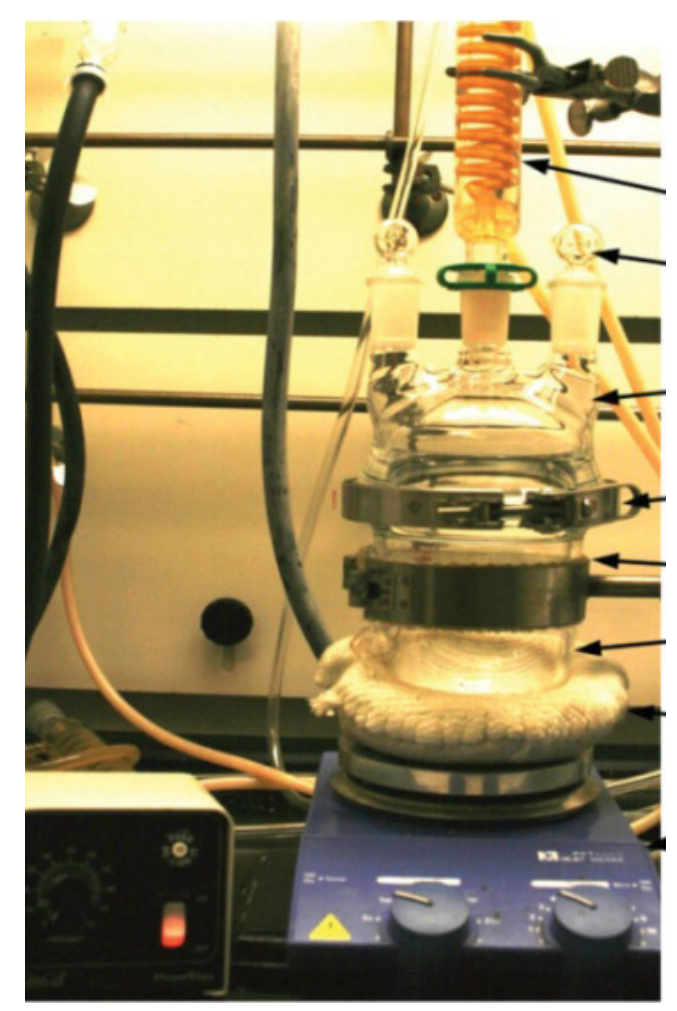

Figure 2.1. Experimental setup of the in situ radical copolymerization

\subsubsection{Experimental setup and conditions}

\subsubsection{Surface characterization}

Fourier transform infrared spectroscopy by attenuated total reflectance (ATR-FTIR) was carried out using a FTIR-4100 spectrometer (Easton, MD) with a maximum resolution of $0.9 \mathrm{~cm}$. A Nanoscope 3A atomic force microscope (AFM) obtained from Veeco 
(Plainview, NY) was employed to measure the thickness of the polymer thin film on the gold chip. Briefly, a $10 \times 10 \mu \mathrm{m}^{2}$ area of the polymer layer was scratched and removed using a contact mode AFM cantilever (spring constant $0.2 \mathrm{Nm}$ ) under the load of $500 \mathrm{nN}$ force. To determine the depth of the scratched area, the morphology of a $30 \times 30 \mu \mathrm{m}^{2}$ area covering the scratched area was imaged by AFM under a $25 \mathrm{~N}$ force load with a scanning speed of 2Hz. A JSM6330F field emission scanning electron microscopy (SEM) from JEOL (Peabody, MA) was employed for observing the surface morphology and component of the LSPR sensing surface.

\subsubsection{Preparation of LSPR measurement}

A BI-2000 SPR instrument was purchased from Biosensing Instrument (Tempe, AZ). A gold SPR chip $(20 \mathrm{~mm} \times 20 \mathrm{~mm} \times 1 \mathrm{~mm})$, which is a BK7 glass slide coated with a $45 \mathrm{~nm}$ layer of gold over a $5 \mathrm{~nm}$ layer of chromium, was mounted on the upper face of the BK7 prism with the gold layer facing upward. A 3-5 $\mu \mathrm{L}$ drop of index matching fluid (World Precision Instruments, Inc., FL) was applied between the glass face of the chip and the prism with care so that no air bubbles were trapped at the interface. Following this, a biocompatible polydimethylsiloxane (PDMS) microfluidic injection chamber gasket with a $5 \mathrm{~mm} \times 1.7 \mathrm{~mm} \times 125 \mu \mathrm{m}$ channel was clamped to the gold face of the chip. A dual syringe pump was attached to the injection chamber allowing both sample and reference buffer flow through the sensing surface. All buffers and solutions were degassed by vigorously stirring and purging with nitrogen before introduction into the injection system to avoid oxidation of ArsA ATPase and ArsD metallochaperone protein by air. Data collection 
and instrument control was performed using the Biosensing Instrument SPR Control Program running on a PC.

In the LSPR experiment, a $670 \mathrm{~nm}$ wave length laser with a $72.2^{\circ}$ incident angle was employed as the light source. The sensing surface was flushed by 50mM MOPS buffer $(\mathrm{pH} 7.5)$ at a $150 \mu \mathrm{L} / \mathrm{min}$ flow rate until a stable SPR baseline was acquired. In general, a 100-150mDeg SPR angle shift would be observed during this process due to the swelling of the PNIPAAm-co-PAAc hydrogel upon introduction of water based MOPS buffer. The MOPS flow rate was then reduced to $50 \mu \mathrm{L} / \mathrm{min}$ for optimized binding time and maintained during the whole LSPR measurement. All measurements were carried out in MPOS buffer environment unless otherwise stated.

In order to demonstrate the enhanced sensitivity by the polymer hydrogel thin layer as the immobilization and sensing material, as well as its suitability for binding kinetic study, ArsA ATPase was coupled to the carboxylic groups of AAc and the specific interaction of As(III) with the immobilized ArsA was observed by LSPR. Initially, carboxylic groups of the polymer layer were activated with a solution of EDC (75mM) and NHS $(15 \mathrm{mM})$ at an injection rate of $20 \mu \mathrm{L} / \mathrm{min}$ (denoted as $\mathrm{X}$ in Scheme 2.1.). ArsA in MOPS with a concentration of $50 \mu \mathrm{g} / \mathrm{mL}$ was injected with a same rate and allowed to react with the activated polymer for $4 \mathrm{~min}$ to form a covalent linkage between the surface carboxylic groups and the ArsA amino-groups. This was followed by an addition of stepped concentration of As(III) to study the binding kinetics and capacity of the ArsA ATPase. Finally, the above experiment was repeated using control chips coated only with AuNPs. 


\subsection{Results and discussion}

\subsubsection{Surface morphology and composition characterization}

To verify the incorporation of NIPAAm and AAc monomeric units in the copolymer grown from the allylmercaptan-modified gold surface, ATR-FTIR spectra were taken from the polymer coated gold chip surface. As a result, Figure 2.1. shows a region of the ATR-FTIR spectrum obtained, which confirms the presence of both NIPAm and AAc in the thin film. The main bands at located at 1651 and $1551 \mathrm{~cm}$ correspond to the carbonyl stretching of the amide group (amide I band) and to the $\mathrm{N}-\mathrm{H}$ stretching of the secondary amide (amide II band), respectively. On the other hand, the appearance of a much less intense absorption band at $1716 \mathrm{~cm}$, characteristic of the carboxyl group, indicates the incorporation of a minor acrylic acid fraction as expected. Besides, a band at $1460 \mathrm{~cm}$ is clearly observed, which can be attributed to the $-\mathrm{CH} 3$ and $-\mathrm{CH} 2-$ deformation of both monomeric units. ${ }^{74}$ 


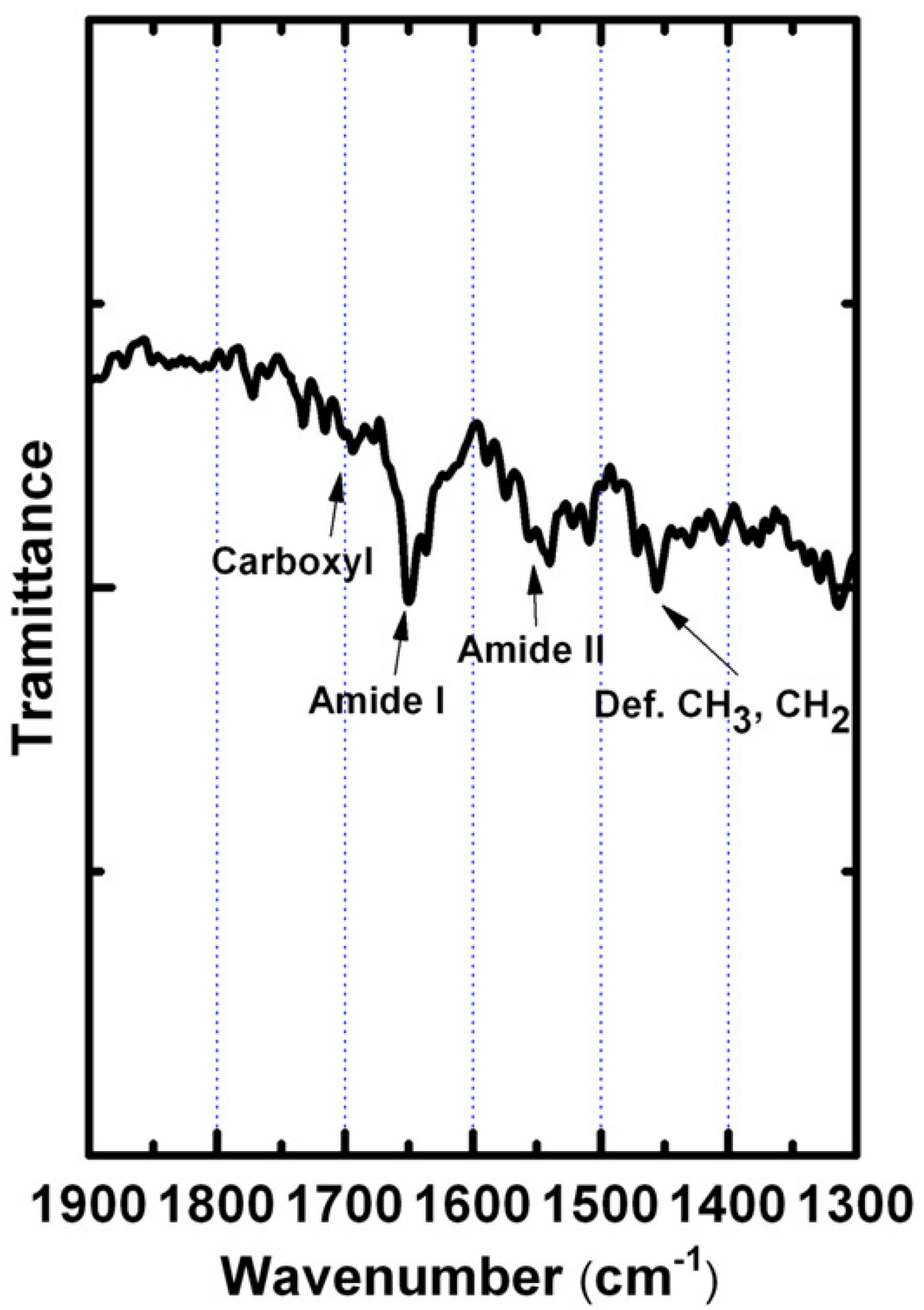

Figure 2.2. FTIR-ATR spectrum of the 3D PNIPAAm-co-PAAc polymer matrix on the sensing surface.

The thickness of the PNIPAAm-co-PAAc polymer layer under hydrated and dehydrated condition was determined by AFM using the aforementioned manner. ${ }^{75}$ Figure 2.2(A) 
shows the morphologies and line profiles of the scratched areas under both conditions, with the average thickness of each sample determined from the line profiles. The hydrated polymer layer displays a thickness of $40.6 \pm 3 \mathrm{~nm}$, where as the dehydrated polymer layer exhibited a thickness of $10.1 \pm 1 \mathrm{~nm}$. Fig. 2.2(B) shows a SEM image in which AuNPs (bright dots) can be observed along the backbone of the polymer hydrogel, indicating that AuNPs were successfully embedded in the PNIPAAm-co-PAAc hydrogel by the linkage with AAc.

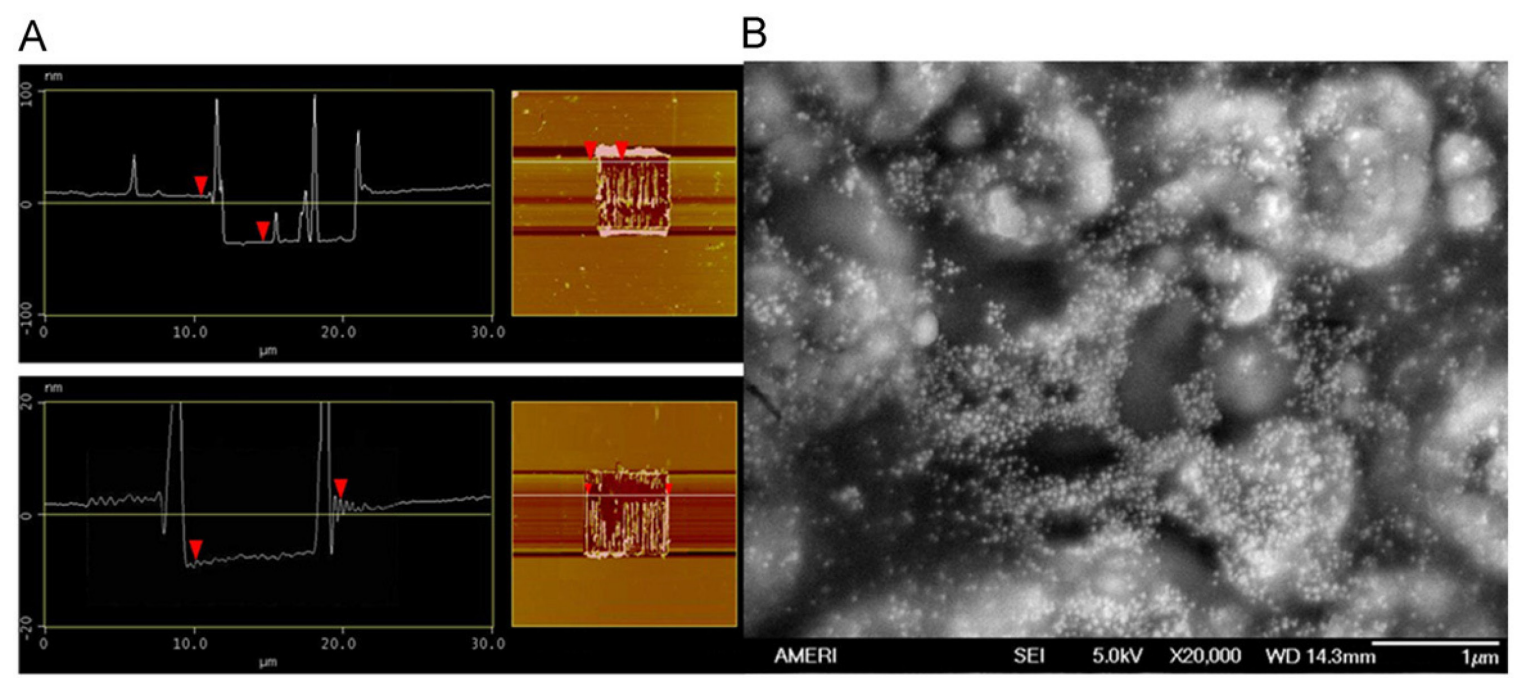

Figure 2.3. (A) AFM images of the polymer matrices under hydrated (upper) and dehydrated (lower) conditions, indicating the swelling-shrinking process. (B) SEM image of the LSPR sensor coated with AuNPs doped hydrogel thin film.

\subsubsection{Optimization of ArsA immobilization conditions}

The incubation time required for the binding reaction between ArsA and polymer matrix is a critical parameter that determines the performance of our kinetics sensor. Insufficient incubation time causes low coverage of ArsA on the surface and leads to lower sensitivity, whereas over-long incubation time may results in multiple layers of ArsA and leads to blockage of binding sites and lower efficiency. To optimize the time required for 
completeness of the covalent linking, $80 \mu \mathrm{g} / \mathrm{mL}$ ArsA solution was injected and past the PNIPAAm-co-PAAc modified gold sensing surface at various injection rates $(5,10,15$, $20,25,30,35 \mu \mathrm{L} / \mathrm{min})$. LSPR angle shift was used to evaluate the surface coverage. The experiment was repeated 3 times with each injection rate. For each injection rate, 3 measurements were averaged as shown in Figure 2.3(A) with standard deviation. This study found that ArsA immobilization with a sample injection rate of $20 \mu \mathrm{L} / \mathrm{min}$ or slower exhibited a stable response demonstrating the complete interaction between ArsA and PNIPAAm-co-PAAc. Therefore, we employed optimized sample injection rate of $20 \mu \mathrm{L} / \mathrm{min}$ for further immobilization and analysis.

In order to evaluate the amount of ArsA ATPase immobilized on the surface of the 3D polymer matrix, we investigated the LSPR response to different concentrations of ArsA injections. Initially, polymer modified LSPR chips were activated using 75mM EDC and $15 \mathrm{mM}$ NHS in MOPS buffer. Following activation, each activated sensor was employed to react with different concentrations of ArsA solution $(10,20,30,40,50,60,70$, $80 \mu \mathrm{g} / \mathrm{mL}$ ) at an injection rate of $20 \mu \mathrm{L} / \mathrm{min}$. The experiment was repeated 3 times with each ArsA concentration. The resulting averaged LSPR response for each concentration was plotted against the corresponding concentration of ArsA with standard deviation as shown in Figure 2.3(B). It is evident that the amount of ArsA ATPase bound to the sensing surface reaches a maximum at $50 \mu \mathrm{g} / \mathrm{mL}$ and remains constant at all concentration beyond. As a result, we employed $50 \mu \mathrm{g} / \mathrm{mL}$ as an optimum concentration for further binding study. 

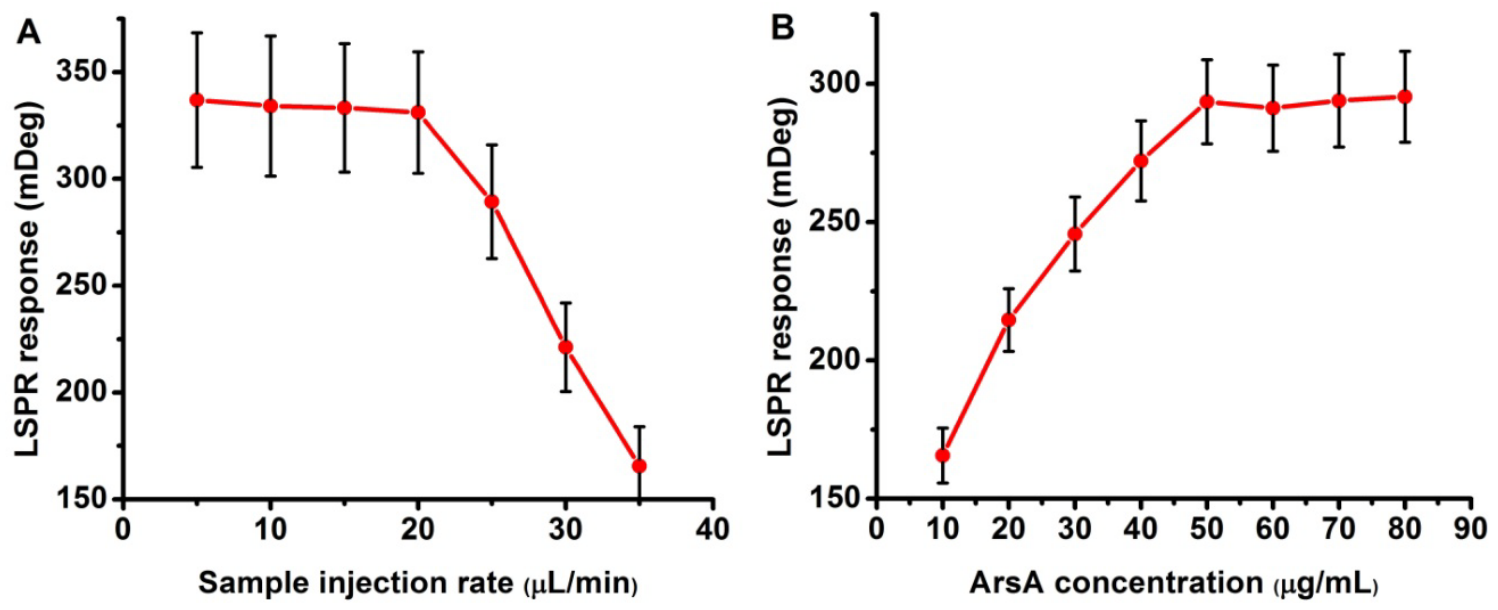

Figure 2.4. Optimization of immobilization conditions: (A) ArsA immobilization on the 3D polymer coated surface with different injection rate $(5,10,15,20,25,30,35 \mu \mathrm{L} / \mathrm{min})$. Maximum SPR angle shift is plotted versus responding injection rate. (B) Different concentration of ArsA $(10,20,30,40,50,60,70,80 \mu \mathrm{g} / \mathrm{mL})$ immobilization on the 3D polymer coated LSPR sensor with optimal injection rate $(20 \mu \mathrm{L} / \mathrm{min})$. Maximum SPR angle shift is plotted versus ArsA concentration.

\subsubsection{ArsA and As(III) interaction}

Sensitivity study was first performed with chips coated by AuNPs doped polymer layer.

Prior to the binding test for each different concentrations of arsenite, ArsA was immobilized onto the activated carboxylic groups by the aforementioned protocol. Then, three different concentrations of arsenite $(8 \mathrm{mM}, 4 \mathrm{mM}, 2 \mathrm{mM})$ were dosed separately with an injection rate of $20 \mu \mathrm{L} / \mathrm{min}$. Figure 2.4(a) shows the LSPR response to the injection of each arsenite sample. When the test solution was introduced at $\sim 50 \mathrm{~s}, \sim 620 \mathrm{~s}$ and $\sim 1100 \mathrm{~s}$, a blue shift of $25.8 \mathrm{mDeg}, 19.1 \mathrm{mDeg}$ and $16.5 \mathrm{mDeg}$ were observed corresponding to the introduction of $8 \mathrm{mM}, 4 \mathrm{mM}$ and $2 \mathrm{mM}$ arsenite sample, respectively. The $2 \mathrm{D}$ AuNPs structure was not expected to induce as much LSPR enhancement as the 3D AuNPs doped polymer matrix according to our previous mentioned theory. Therefore, we repeated the binding experiment with chips decorated by 2D AuNPs structure. Figure 
2.4(b) indicates that a blue shift of $3.7 \mathrm{mDeg}, 2.9 \mathrm{mDeg}$ and $2.4 \mathrm{mDeg}$ were recorded in response to the same concentration gradients of arsenite. The above experiments were repeated 4 times for both 3D and 2D LSPR sensors. Similar results were observed (data not shown). As a result, the signal enhancement of the 3D polymer matrix was calculated to be about 6.8 fold (averaged from 4 repeated comparisons). This sensitivity increase can be attributed to the unique swelling-shrinking property of the PNIPAAm-co-PAAc hydrogel, yielding a greater capacity for ArsA immobilization than that of the 2D AuNPs chip (Scheme 2.1.).

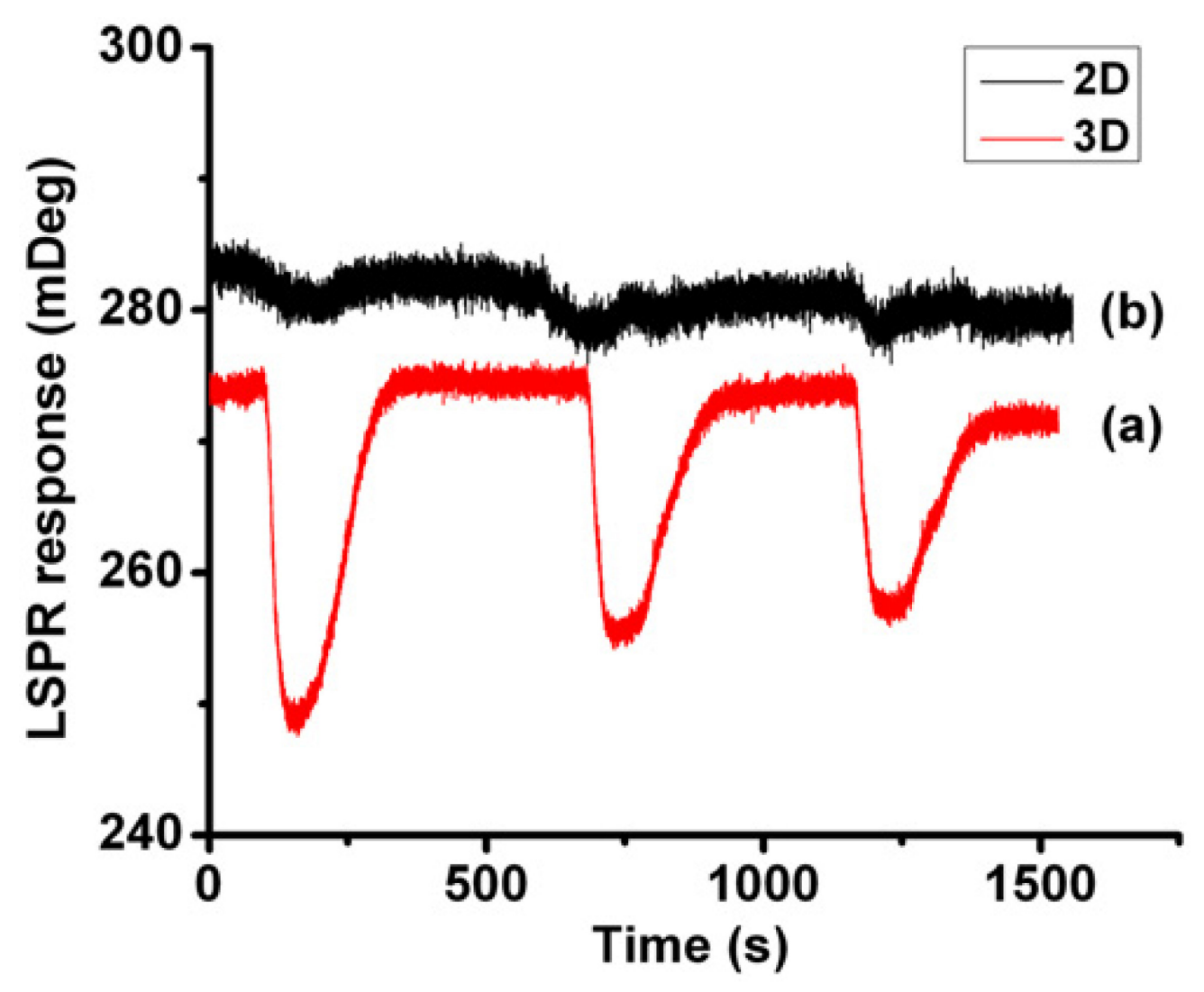

Figure 2.5. SPR sensorgrams for the covalently immobilized ArsA interacting with As(III) obtained with (a) 3D AuNPs doped polymer coated sensor and (b) 2D AuNPs structure modifiedsensor. 
We expected larger enhancement from the 3D polymer matrix since the previous mentioned AFM measurements demonstrate that the thickness of the hydrated polymer is $40.615 \mathrm{~nm}$, indicating an ideal capacity increase of 180 fold for ArsA (63kDa, a=73.34, $b=75.64, c=223.42 \AA$ ) compared to the ArsA monolayer formed on the $2 \mathrm{D}$ chip. One possible explanation for this discrepancy is the heterogeneous distribution of carboxylic groups during the random copolymer reaction resulting in a lack of available binding sites for ArsA. We also suspect that the injected ArsA bound to the surface of the polymer matrix has blocked the access of the unbound ArsA ATPase to binding sites inside the polymer matrix.

\subsubsection{Kinetics study of ArsA-As(III) binding}

ArsD is a metallochaperone that delivers As(III), as well as other trivalent metalloids, to the ArsA ATPase. Previous studies have shown that interaction with ArsD increases the affinity of ArsA for As(III), conferring resistance to environmental concentrations of arsenic. $^{76}$ However, there is a lack of research on the binding affinity of ArsA for ArsD till now. Based on measurements on the effect of the As(III) chelator dimercaptosuccinic acid on the transfer reaction, it is suggested that As(III) transfer is directly channeling from one protein to the other, rather than dissociating from ArsD and reassociating with ArsA. Therefore, affinity study between ArsA and ArsD is essential to understanding the structure of ArsA-ArsD complex and improve understanding of the transfer reaction.

Initially, ArsA was immobilized to the activated carboxylic groups of the polymer as previously mentioned. Following this, 1\% ethanolamine was injected to block the free 
carbonyl groups. ArsD metallochaperone $(50 \mu \mathrm{g} / \mathrm{mL})$ was then introduced to the flow chamber at $20 \mu \mathrm{L} / \mathrm{min}$. For comparison, ArsD was substituted by bovine serum albumin (BSA) in the negative control experiment. Figure 2.5 shows the LSPR sensorgram for the ArsA-ArsD interaction study. The binding between ArsA and ArsD (red) results in an angle shift of $356.2 \mathrm{mDeg}$, whereas the binding between ArsA and BSA (black) only results in an angle shift of $45.5 \mathrm{mDeg}$. The above experiments were repeated 4 times for both ArsD and BSA binding. Similar results were observed (data not shown). Thus, it is evident that ArsA is able to specifically bind ArsD without the presence of As(III) and MgATP. This result is consistent with the direct transfer of As(III) in the ArsA-ArsD complex.

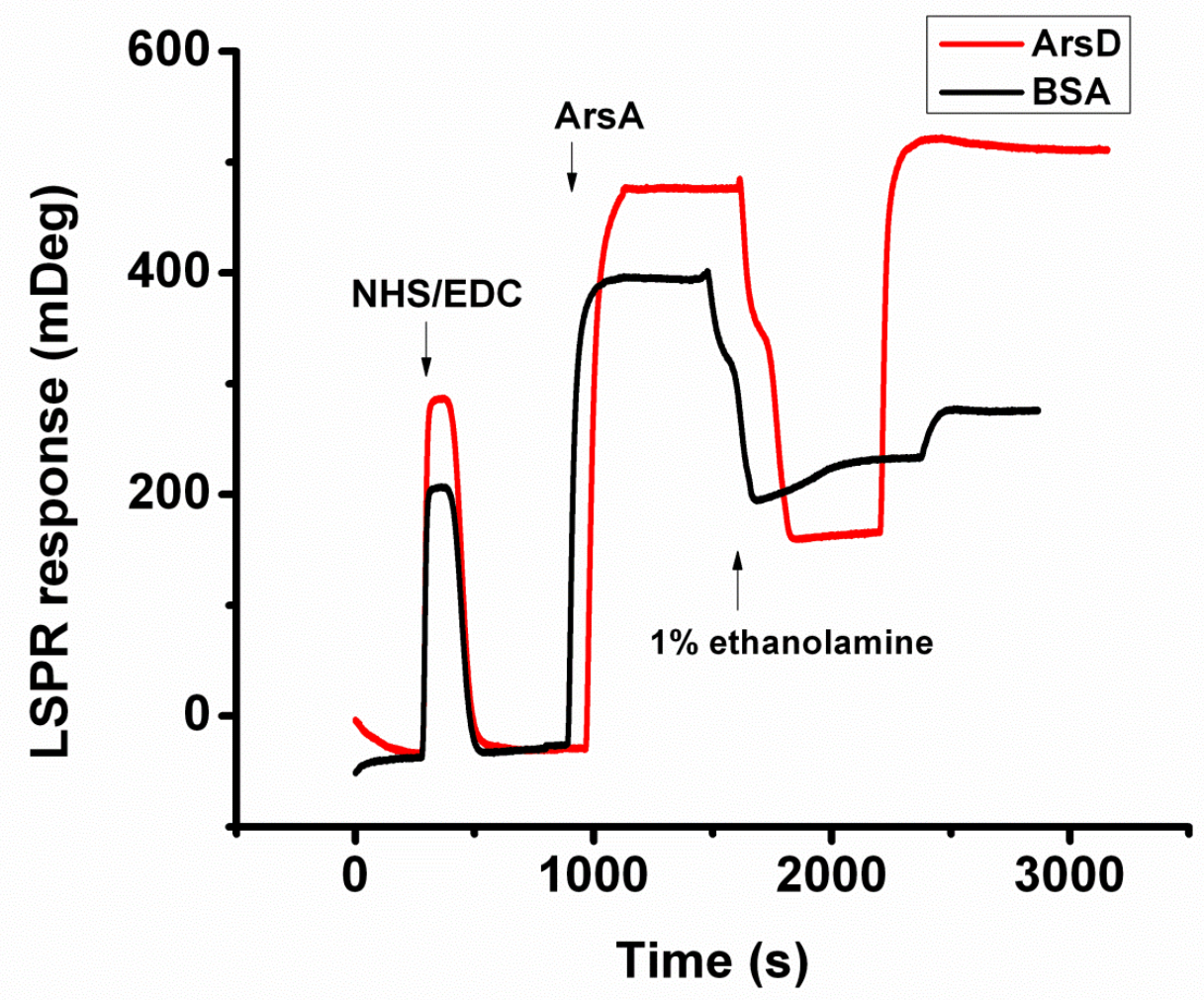

Figure 2.6. SPR sensorgrams for covalently immobilized ArsA interacting with (red line) ArsD and (black line) BSA as control. 


\subsubsection{Effects of ArsD and MgATP on ArsA-As(III) binding}

We have previously shown that ArsD transfers $\mathrm{As}(\mathrm{III})$ and $\mathrm{Sb}(\mathrm{III})$ to ArsA in the presence of MgATP. ${ }^{77}$ Herein, binding kinetics of ArsA-As(III) interaction was compared using the 3D LSPR sensing platform under MgATP catalytic, ArsD promotion, and noncatalytic conditions.

ArsA was bound to the 3D LSPR sensing surface using the same manner. As(III) samples of 10 different concentrations $(0.1-1 \mathrm{mM})$ were prepared in MOPS buffer. First, ArsA-As(III) binding kinetics was measured without catalysis as a control experiment. Samples with $0.1-1 \mathrm{mM}$ As(III) were injected in 10 min interval between each other to allow for baseline stabilization. Following this, ArsA-As(III) binding kinetics measurement was repeated under the presence of ArsD or MgATP, respectively. The measurements were repeated 4 times for each condition. SPR angle shift-time curve (data not shown) was obtained for each measurement in realtime. In this experiment, the surface interaction $[\mathrm{ArsA}+\mathrm{As}(\mathrm{III}) \rightarrow \operatorname{ArsA}-\mathrm{As}(\mathrm{III})]$ was measured by the rate of ArsAAs(III) complex production. Such interaction can be described as a second order reaction:

$A+B \longrightarrow P$

by the pseudo-second-order kinetic equation:

$$
\text { Rate }=k[A][B .
$$

where $\mathrm{k}$ is a second order rate constant, $[\mathrm{A}]$ and $[\mathrm{B}]$ are the concentrations of reactants $\mathrm{A}$ and $\mathrm{B}$, respectively. 
In the association phase of the SPR sensorgram, we used the modified pseudo-secondorder kinetic equation to describe the reaction:

$$
\max \left(\frac{\mathrm{dR}}{\mathrm{dt}}\right)=\mathrm{ka}[\operatorname{Ars} \mathrm{A}][\operatorname{As}(\mathrm{III})]
$$

where $\mathrm{R}$ is the SPR signal at time $\mathrm{t}, \mathrm{k}_{\mathrm{a}}$ is the association rate constant which indicates the binding affinity between two reactants, $[\mathrm{ArsA}]$ and $[\mathrm{As}(\mathrm{III})]$ are the concentrations of ArsA and As(III), respectively. The maximum value of $(\mathrm{dR} / \mathrm{dt})$ for each sample was determined by calculating the maximum slope of the association curve using Matlab. Linear fitting was utilized to obtain $\mathrm{k}_{\mathrm{a}}$ since $[\mathrm{ArsA}$ ] is constant. Figure 2.6(A) shows the averaged $\max (\mathrm{dR} / \mathrm{dt})$ for each $\mathrm{As}(\mathrm{III})$ concentration with standard deviation. After linear fitting, $\mathrm{k}_{\mathrm{a}}$ was calculated to be $1.07 \mathrm{M} / \mathrm{min}$ with a 0.06 standard error for noncatalytic ArsA-As(III) interaction. Based on the aforementioned study, interaction with ArsD increases the affinity of ArsA for As(III). Therefore, association rate constant was also determined for the ArsA-As(III) interaction under the presence of ArsD in the environmental buffer and $\mathrm{k}_{\mathrm{a}}$ was calculated to be $2.79 \mathrm{M} / \mathrm{min}$ with a 0.41 standard error using the same method, as shown in Figure 2.6(B). It appears that the $\max (\mathrm{dR} / \mathrm{dt})$ vs. As(III) concentration plot fits a quasi-exponential shape under the presence of ArsD, which does not agree with the linear fitting of our model. Such mismatch can be attributed to the unexpected change of the surface ArsA concentration. Due to the comformational and functional similarity between ArsA and ArsD, it is likely that the ArsD in the flowing buffer affected the binding equilibrium between the surface and ArsA. Furthermore, ArsA contains two nucleotide binding sites (NBSs) and a binding site for arsenic, and crystallizes in the presence of As(III) and MgATP. ${ }^{78}$ Based on these 
structural features, we tested association rate constant under the MgATP catalysis. Results show that the $k_{a}$ was significantly raised to $16.09 \mathrm{M} / \mathrm{min}$ with a 0.66 standard error (Figure 2.6(C)). By comparing these three sets of experiments, it is obvious that the binding efficiency is significantly raised under the presence of $\mathrm{MgATP}$, indicating that the activity of the arsenic binding site is dependent on the binding status of the two NBSs for ATP. ${ }^{79}$ Based on the result, As(III) transfer occurs only under conditions where ArsA hydrolyzes ATP, suggesting that ArsD transfers As(III) to an ArsA conformation transiently formed during catalysis and not simply to the closed conformation that ArsA adopts when As(III) and MgATP are bound.

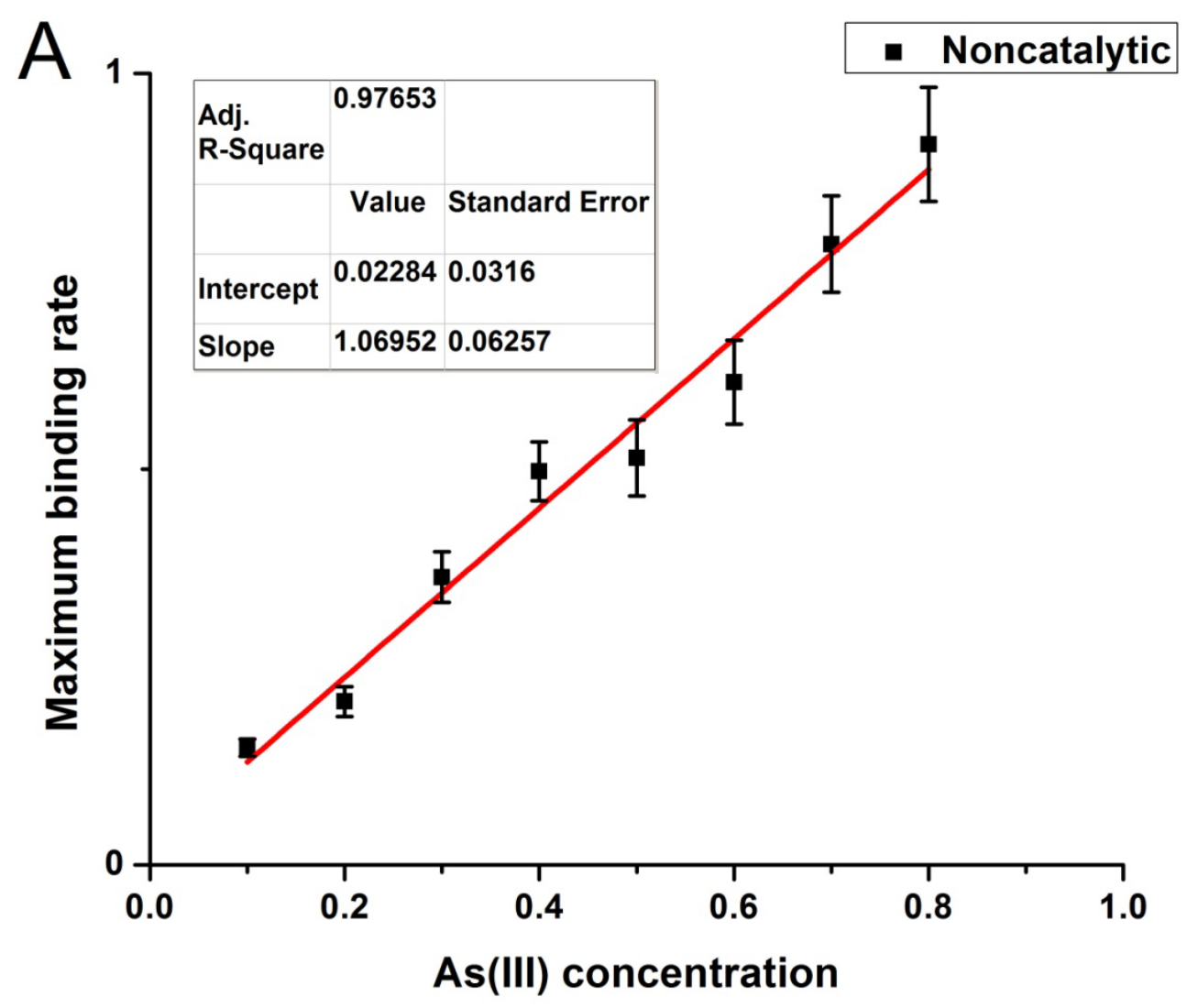




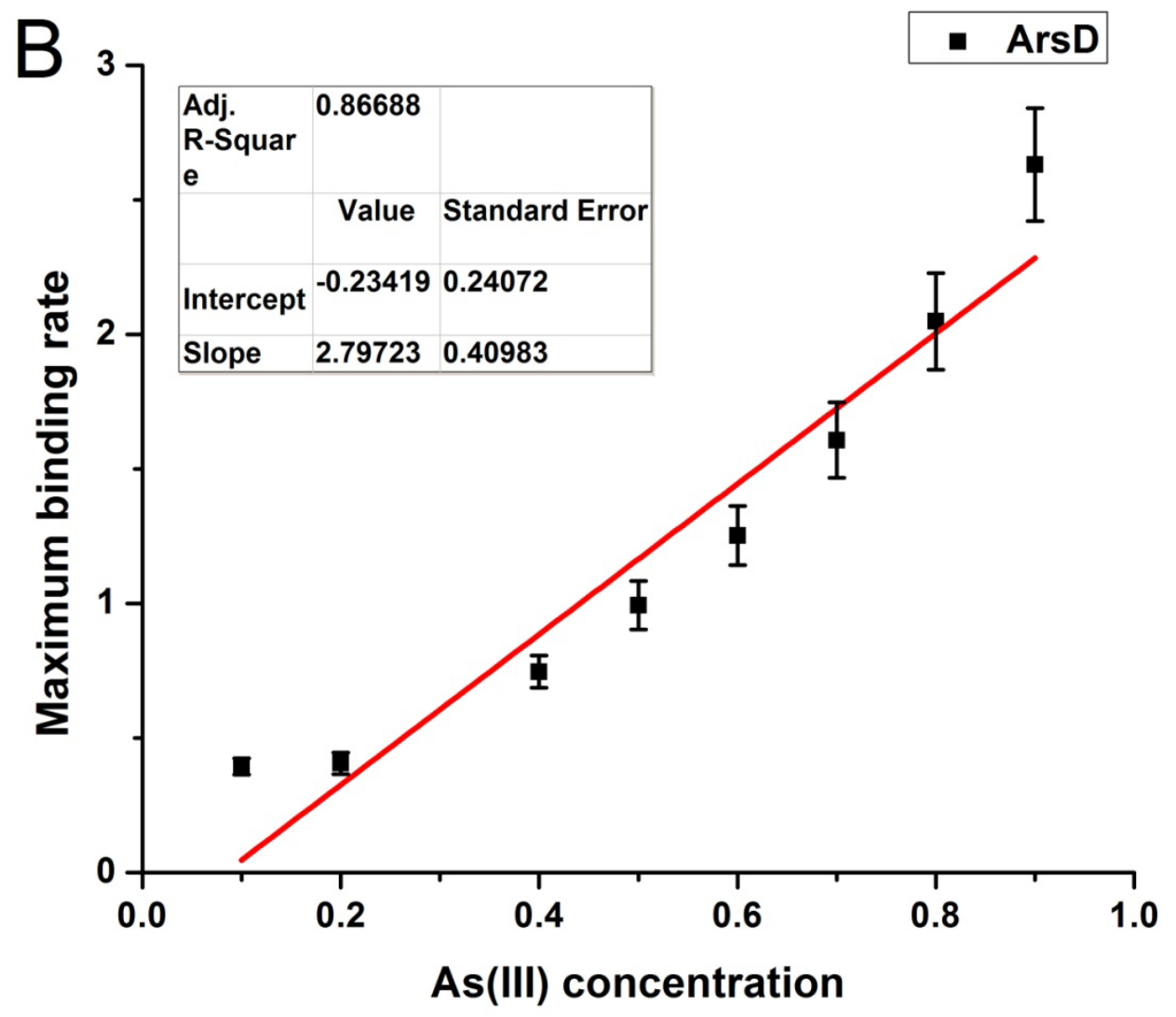




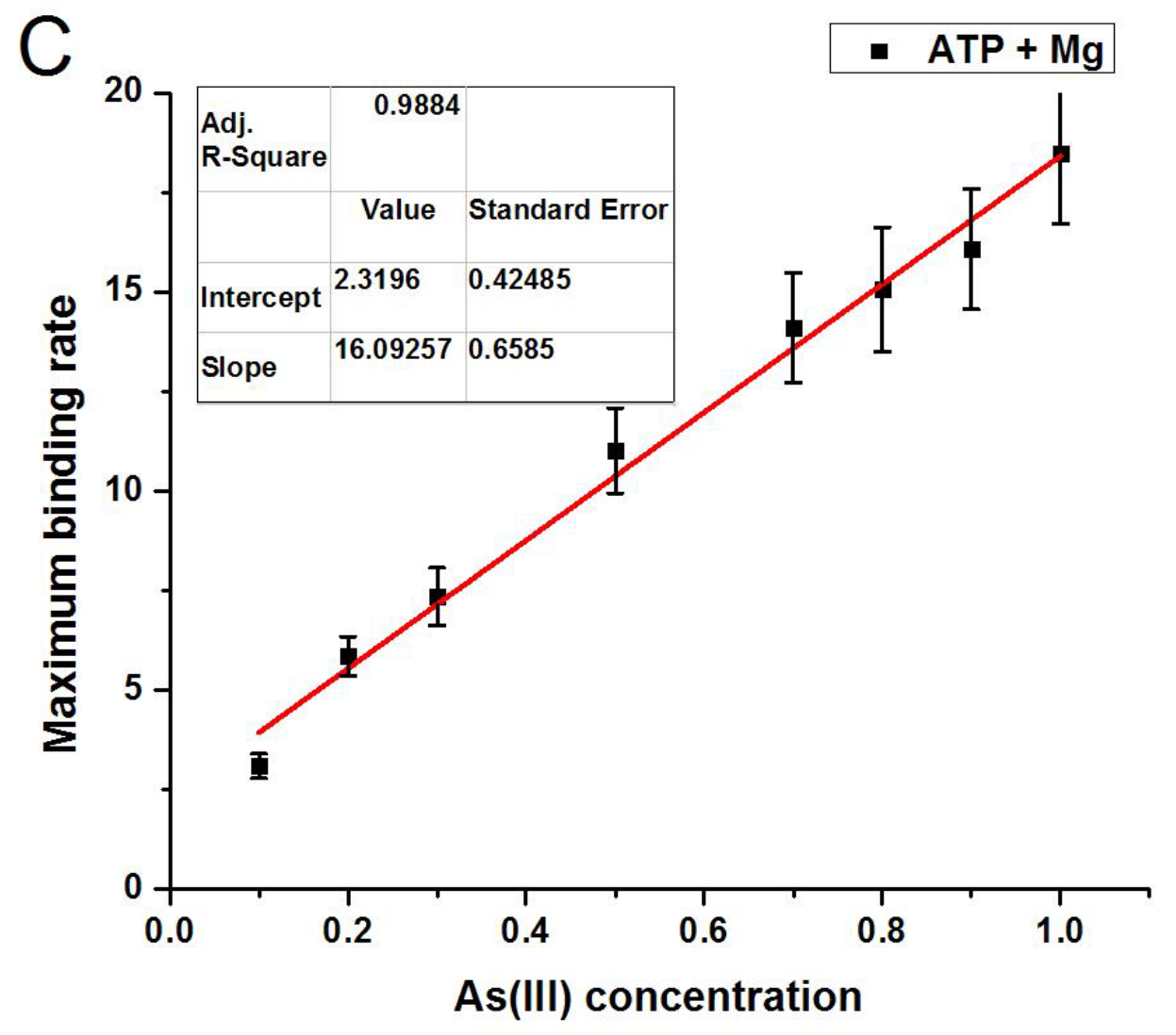

Figure 2.7. Comparison of ArsA-As(III) association rate constant (slope) under the effect of different binding status. Figure showing: linear fitting curve of the relationship between maximum binding rate and As(III) concentration under (A) noncatalytic, (B) ArsD bounded and (C) MgATP catalyzing conditions of ArsA.

\subsection{Conclusions}

We have demonstrated the fabrication of AuNPs decorated 3D PNIPAAm-co-PAAc hydrogel modified LSPR sensors. Sensitivity enhancement of 7 fold was achieved for measurement of changes in bulk refractive index using LSPR spectroscopy, compared to the conventional 2D nanostructural LSPR sensor. Not only can the 3D hydrogel matrix act as a molecular sponge to increase the analyte absorbing capacity, but it also provides specificity, tunable size of porous structure and interparticle distance for different potential applications. Furthermore, we have demonstrated the ability of ArsA ATPase 
specifically bind ArsD metallochaperone without presence of $\mathrm{As}(\mathrm{III})$ and $\mathrm{MgATP}$, thus proving the existence of the structure of ArsA-ArsD complex, which indicates direct channeling of As(III) from ArsD to ArsA. We also applied the 3D LSPR sensor to study the binding kinetics of the arsenic detoxifying systems found in E. coli. Results indicates that transfer of As(III) from ArsD to ArsA is dependent on the binding and hydrolysis of $\operatorname{MgATP}$.

Overall, this work presents a novel combination of a highly sorbtive polymer with AuNPs for enhanced sensitive LSPR spectroscopy. Real-time binding kinetics data were obtained by the LSPR sensor for further understanding of a biochemical process. The flexibility of this sensing platform to accommodate different ligands provides a practical strategy to detection and kinetic study of other biomolecules. 


\section{Chapter 3}

Sensing platform upgrade for biomarker studies from a living cell model 


\subsection{Introduction}

SPR based biosensing has been a very hot topic drawing substantial research interests in the past decade. Promising biomedical applications of SPR have also been widely studied such as detection of binding activity between cells, proteins, DNA and even small inorganic molecules. ${ }^{80-84}$ In the previous chapter, a LSPR with enhanced sensitivity for detecting bindings between proteins and heavy metal molecules was successfully achieved. However, this sensing platform is only capable for detection of analytes in solution. An upgraded SPR sensing platform with the ability of direct measurement of cellular secretion (proteins, DNA, RNA, etc.) from living cells is needed for both fundamental and clinical research.

The principle of SPR biosensors is the measurement of refractive index changes at a plane interface between two media with dielectric constants of opposite signs, a dielectric and a metal, such as gold. SPR can be excited when a wedge of polarized light is directed towards the glass face of the sensor surface under the condition of total internal reflection. The resonant angle at which a minimal intensity of reflected light occurs is a function of the local refractive index at or near the gold surface. Such refractive index changes associate intimately with the adsorption or desorption of molecules from the surface, and thus one can expect its great potential in biorecognition measurements. ${ }^{53}$ This is a newly emerged technique for biomarker detection that is sensitive, fast and realtime. To date, all previously established SPR based sensing platforms have been limited to detection of analyte in a prepared sample. ${ }^{19,85-86}$ In these strategies, collection of analytes from cell culture media, purification and pretreatment of analytes are usually 
required for the purposes of cellular exocytosis and cellular signaling pathways studies. ${ }^{87-}$ 88 These redundant steps are time consuming, and also introduce unpredictable errors to the experiments. Therefore, it is desirable to find an alternative method for direct measurement of secretions from living cells.

Among all types of cellular secretions, biomarkers are no doubt the most significant ones for clinical, medical and biochemical applications. According to the FDA's definition, there are four different types of biomarkers: (1) Prognostic biomarkers are used to determine the disease progression in a patient; (2) Predictive biomarkers are a baseline characteristic that indicate the likelihood a specific patient will respond to a given treatment; (3) Pharmocodynamic biomarkers test the biological response that occurres in a patient after receiving a particular drug, such as a change in blood pressure or cholesterol. Drug companies usually test these biomarkers in phase II clinical trials and submit the results to the FDA; (4) Surrogate endpoints biomarkers are used as a substitute for a clinical efficacy endpoint.

Biomarker based cancer diagnosis and treatment on the molecular level have emerged recently. Compared to traditional antineoplastic solutions, such as chemotherapy, radiation therapy, and cryosurgery etc., biomarker therapy revealed mild side effects in clinical studies. ${ }^{89}$ Biomarkers comprise of cellular molecules such as proteins or nucleic acids that can be detected in cells, blood, urine, or other body fluids and are overexpressed due to the onset of disease. Proteomic biomarkers are widely involved in the development of many types of cancer. Like all other types of human cells, cancer cells 
also rely on a constant oxygen supply to maintain their cellular activity. A tumor larger than a millimeter will starve itself of oxygen and energy unless new blood vessels are built to provide a supply. For this reason, many cancer cells employ the normal processes of angiogenesis in order to build their own blood supply. ${ }^{43}$

VEGF, a 43 to $46 \mathrm{kDa}$ glycoprotein, is a widely studied angiogenic signal protein biomarker produced by oxygen-hungry cells to promote the growth of blood vessels. ${ }^{90}$ It binds to specialized receptors on the surfaces of endothelial cells and directs them to build new vessels. Some types of tumor cells produce abnormally large amounts of VEGF or block the action of angiogenesis inhibitors. This action is termed as "angiogenic switch", giving the ability of metastasis to the tumor, since a custom-made blood supply can be constructed wherever new tumors begin to grow. ${ }^{44}$

In this work, we report a new concept of a SPR biosensing system for realtime VEGF secretion study. A novel design by integrating a mini cell culture module to the SPR system will be introduced. Unlike the traditional configuration of SPR systems for biomarker detection, living cells are cultured on the ceiling of a customized SPR flow cell chamber, and biomarker secretion from cells is rapidly monitored by an immune SPR sensing device (Scheme 3.1). As a model system, the SKOV-3 ovarian cancer cell line is used to demonstrate VEGF secretion lifetime measurement. To the best of our knowledge, this new SPR based biosensing strategy for direct measurement of biomarker from living cells has not been reported previously. 


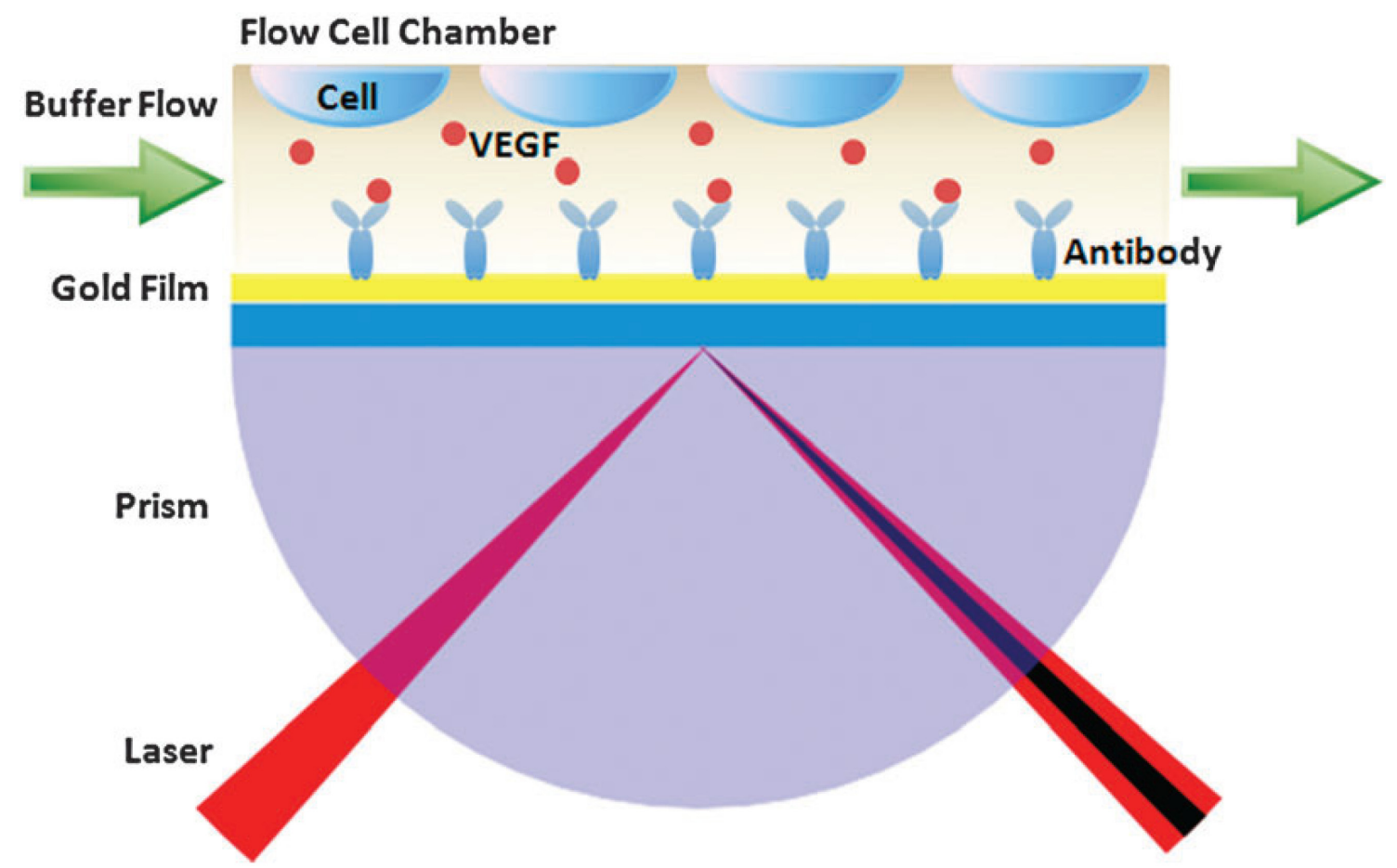

Scheme 3.1 Configuration of the SPR based biosensor integrating a mini cell culture module for direct measurement of biomarker from living cells.

\subsection{Experimental}

\subsubsection{Materials}

Human ovarian carcinoma cell line (SKOV-3), McCoy's 5A medium, and fetal bovine serum were purchased from American Type Culture Collection (Manassas, Virginia). 6well tissue culture plates, d-poly coverslips, and formalin were purchased from Fisher Scientific. VEGF, monoclonal anti-VEGF antibody, KREB's buffer and Penicillin were obtained from Sigma-Aldrich (St. Louis, MO). MUA was purchased from Asemblon (Redmond, WA). Calcium Ionophore (A23187) was purchased from Acros Organic (New Jersey). EDC and NHS were obtained from Alfa Aesar (WardHill, MA). Protein G was purchased from Fisher Scientific (Waltham, MA). All reagents and solvents were used as received unless otherwise stated. 


\subsubsection{Instrumentation}

A BI-2000 SPR instrument purchased from Biosensing Instrument (Tempe, AZ) was used for data collection. A $670 \mathrm{~nm}$ wave length laser with a $72.2^{\circ}$ incident angle was employed as the light source of the SPR instrument. The gold SPR chip $(20 \mathrm{~mm} \times 20 \mathrm{~mm} \times 1 \mathrm{~mm})$ is a BK7 glass slide coated with a $45 \mathrm{~nm}$ layer of gold over a $5 \mathrm{~nm}$ layer of chromium. A 3-5 $\mu \mathrm{L}$ drop of index matching fluid (World Precision Instruments, Inc., FL) was to be applied between the glass face of the chip and the prism with care so that no air bubbles were trapped at the interface. Following this, a biocompatible PDMS microfluidic injection chamber gasket with a $5 \mathrm{~mm} \times 1.7 \mathrm{~mm} \times 125 \mu \mathrm{m}$ channel was clamped to the gold face of the chip. A dual syringe pump was attached to the injection chamber allowing both sample and reference buffer flow through the sensing surface. An Olympus IX81 fluorescence microscopy (Japan) with a 20x objective was employed for cell fluorescent imaging. A CCD camera was used to capture the signals and the images were software-merged with pseudo color.

\subsubsection{Surface treatment for SPR flow chamber}

SPR flow chamber gasket (Figure 3.1) was detached from the SPR flow cell. Several drops of $0.1 \% \mathrm{w} / \mathrm{v}$ gelatin solution made by boiling distilled water were applied onto the PDMS gasket to cover the whole surface. The gasket was then dried for $12 \mathrm{~h}$ in a biological fume hood to prevent contamination. A cell culture petri dish and a uncoated PDMS gasket were also prepared as positive and negative control substrates, respectively. 


\section{Retainer Ring \\ Flow Injection \\ Gasket \\ Flow Cell
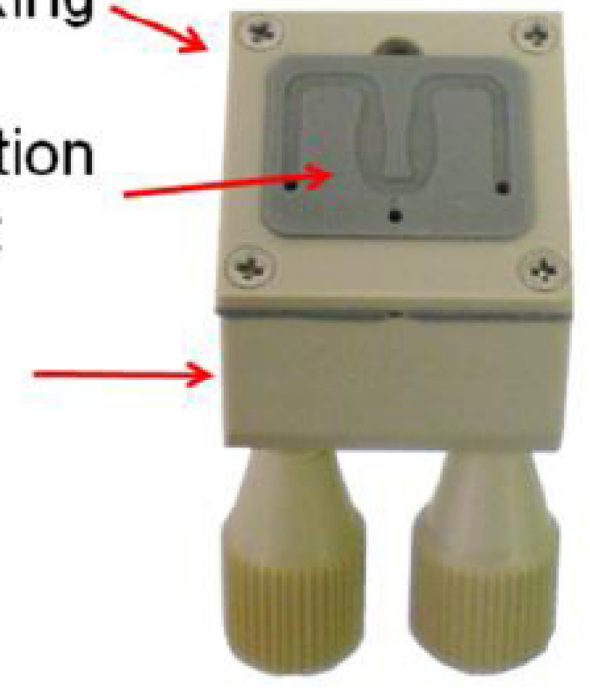

Figure 3.1. SPR Flow Cell and Flow Chamber Gasket.

\subsubsection{Human cell culture}

SKOV-3 cells were cultured in McCoy's 5A medium added with 1\% penicillin and 10\% fetal bovine serum, and kept in a $37^{\circ} \mathrm{C}$ cell incubator with a humidified atmosphere of $5 \% \mathrm{CO}_{2}$ and $95 \%$ air. 200,000 cells were seeded on the gelatin coated SPR flow chamber gasket and kept in a 6-well tissue culture plates with cell culture media for $48 \mathrm{~h}$ before each experiment. The same cell culture was repeated on an uncoated PDMS gasket and a tissue culture plate as control experiments.

\subsubsection{Cell stain and fluorescent imaging}

$200 \mu \mathrm{M}$ MitoTracker Red CMXRos dye stock solution and the $1.0 \mathrm{mM}$ Hoechst 33342 dye were diluted into HBSS or appropriate cell medium with serum. The concentration for MitoTracker Red CMXRos dye is 10-50 nM; The concentration for Hoechst 33342 
dye is $1.0 \mu \mathrm{g} / \mathrm{mL}$. Both dyes may be combined in a single staining solution. Then apply a sufficient amount of labeling solution to cover cells adhering to substrates. Incubate for 15 minutes at $37^{\circ} \mathrm{C}$. When labeling is complete, remove the labeling solution and wash cells twice in cell medium. Labeled cells will then be fixed with $4 \%$ formaldehyde for 15 minutes at $37^{\circ} \mathrm{C}$, followed by washes in buffer and staining with any additional counterstains.

The substrates were then removed and mounted on glass microslides with antifade reagent/mounting medium mixture. Then, the specimens were observed by fluorescence microscopy (Olympus IX81, Japan) with a 20x objective. The fluorescence was imaged at Hoechst channel (nucleus stain dye, ex/em: 358/461 nm), and $\lambda$ ex (570), $\lambda \mathrm{em}(590 \mathrm{~nm}$ ) for mitotracker red. A CCD camera was used to capture the signals and the images were software-merged with pseudo color. The fluorescence microscope settings were kept the same throughout the experiment with the exception of the exposure time. The images were recorded at the same exposure time for Hoechst and MitoTracker Red.

\subsubsection{Calibration of SPR for VEGF measurements}

A previously reported method was used for immobilization of monoclonal anti-VEGF

antibody on the SPR gold chip. ${ }^{91}$ Briefly, SPR gold chip will be emerged in 1mM MUA (ethanol) solution for $12 \mathrm{~h}$ at $4^{\circ} \mathrm{C}$ to form a layer of carboxylic groups on the gold surface. The gold chip will then be washed with copious ethanol and mounted on the SPR system. Carboxylic groups were then activated with a solution of EDC $(75 \mathrm{mM})$ and NHS $(15 \mathrm{mM})$. Following this, an activated SPR chip was immobilized by injecting $50 \mu \mathrm{g} / \mathrm{mL}$ 
protein G solution to capture the Fc portions of the antibody in order to assure proper antibody orientation. ${ }^{92} 50 \mu \mathrm{g} / \mathrm{mL}$ monoclonal anti-VEGF was bounded on top of the protein G layer as the biorecognition ligand. All injections to the SPR microfluidic system were performed with a $20 \mu \mathrm{L} /$ min rate. SPR experiments were performed in phosphate buffered saline (PBS) as the flowing buffer, and each solution was also prepared in PBS $(\mathrm{pH}=7.4)$ unless otherwise specified. The sensor calibration was performed using VEGF samples with 8 different concentrations $(0.25,0.5,1,1.5,2,2.5$, $3,4 \mu \mathrm{g} / \mathrm{mL}$ ). The calibration was repeated 4 times for reproducibility test.

\subsubsection{Direct measurement of VEGF from living cancer cells}

In order to demonstrate direct measurement of VEGF from living carcinoma cells, SKOV-3 cells were cultured for $48 \mathrm{~h}$ on gelatin coated gaskets using the aforementioned method. The same functionalization protocol described above will be used for immobilization of monoclonal anti-VEGF antibody on the SPR sensing chip. The PDMS flow chamber gasket with SKOV-3 cell culture was then removed from cell culture media and throughly rinsed with Kreb's buffer to remove cell culture media and unattached cells. Following this, the PDMS gasket with cells was mounted on the SPR flow chamber, and SPR flowing buffer was changed from PBS $(\mathrm{pH}=7.4)$ to Kreb's buffer $(\mathrm{pH}=7.4)$ in order to maintain cell viability during experiments. After resuming the buffer flow, $500 \mu \mathrm{m} \mathrm{Ca}^{2+}$ ionophore (A23187) was injected to induce rapid exocytosis of VEGF from the SKOV-3 cells. The above VEGF measurement was repeated 4 times. 


\subsection{Results and discussion}

\subsubsection{Calibration for VEGF}

Initially, reproducibility and linearity of the functionalized $\mathrm{Au}$ sensing surface were examined using different concentrations of the VEGF sample. Figure 3.2.A shows the time resolved SPR spectra in response to VEGF binding $(3,4 \mu \mathrm{g} / \mathrm{mL}$ samples not shown). VEGF binding can be clearly characterized by the SPR angle shift upon introduction of the VEGF sample solution. Figure 3.2.B depicts the calibration curve obtained by a linear fit of SPR response to different concentrations of VEGF samples. The SPR sensor allows real-time and sensitive VEGF detection within a linear dynamic range of 0.1-2.5 $\mu \mathrm{g} / \mathrm{mL}$. For each VEGF concenration, the SPR response was determined by the average of 4 measurements. The maximum value of the inter-assay relative standard deviations was $13.6 \%(n=4)$. This indicates that our detection strategy offers an acceptable reproducibility towards the detection of VEGF. 


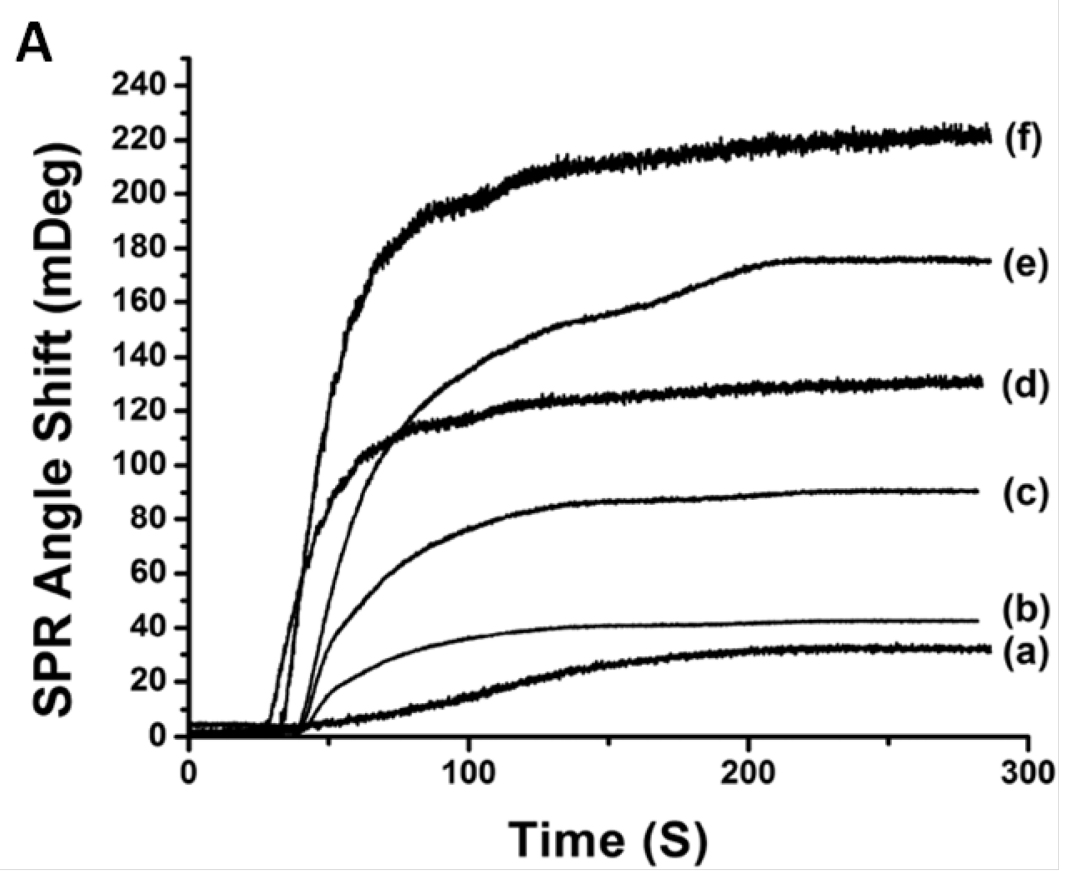

B

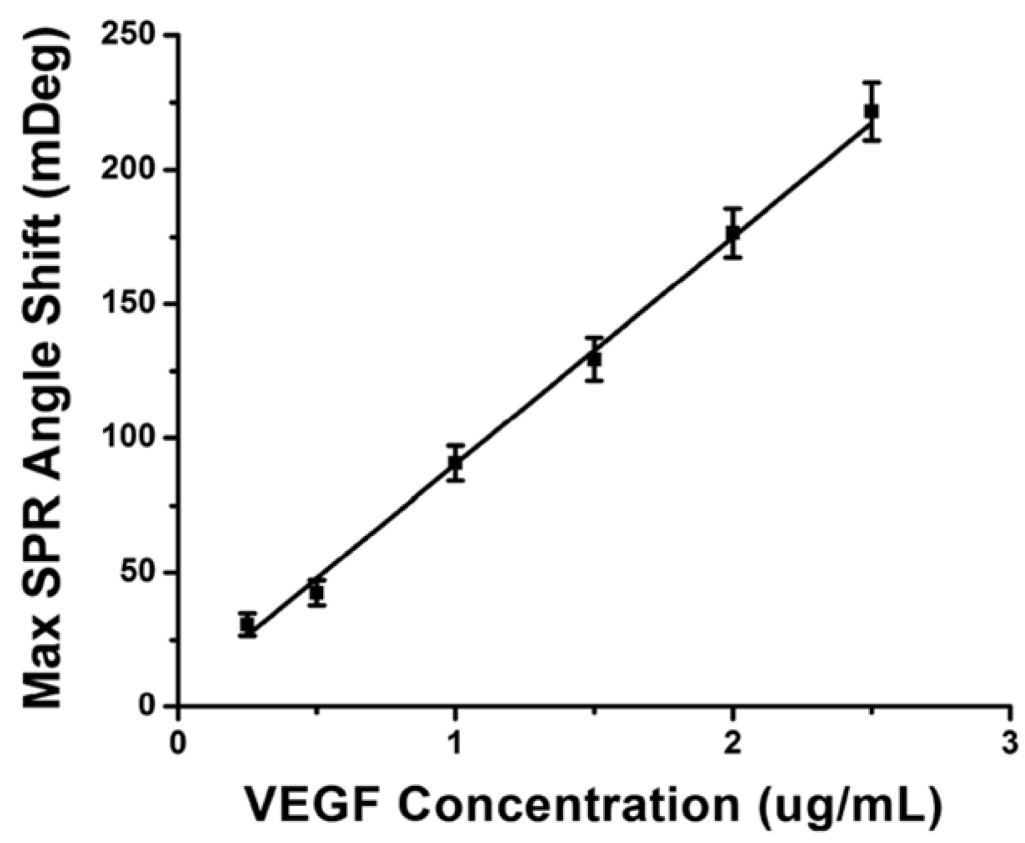

Figure 3.2. (A: upper) SPR sensor responses to VEGF solution at different concentration levels: (a) at $0.25 \mu \mathrm{g} / \mathrm{mL}$, (b) at $0.5 \mu \mathrm{g} \mathrm{mL}$, (c) at $1 \mu \mathrm{g} / \mathrm{mL}$, (d) at $1.5 \mu \mathrm{g} / \mathrm{mL}$, (e) at $2 \mu \mathrm{g} / \mathrm{mL}$ and (f) at $2.5 \mu \mathrm{g} / \mathrm{mL}$. (B: lower) Calibration curve: the linear relationship between change of SPR angle shift and VEGF concentration. 


\subsubsection{Fluorescent imaging}

For the purpose of measuring VEGF secretion directly from living cancer cells, we first demonstrated cell viability in the SPR flow chamber. Fluorescent imaging was performed using a previously reported method in order to examine cell confluency on each substrate. ${ }^{93}$ As shown in Figure 3.3, cell counts on the tissue culture plate, gelatin coated gasket and uncoated gasket are 174, 218 and 76, respectively. Since the gelatin coated gasket showed significant enhancement of cell attachment compared to the uncoated gasket, it is a suitable substrate for the living cell experiment in the SPR flow chamber.

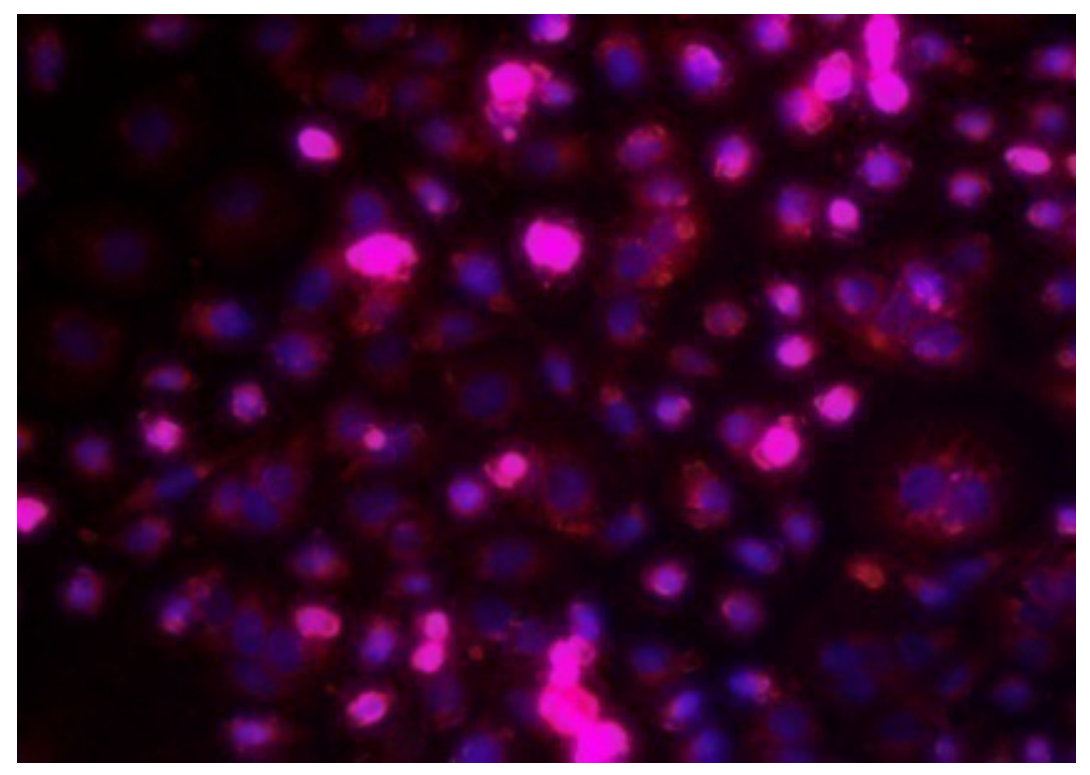



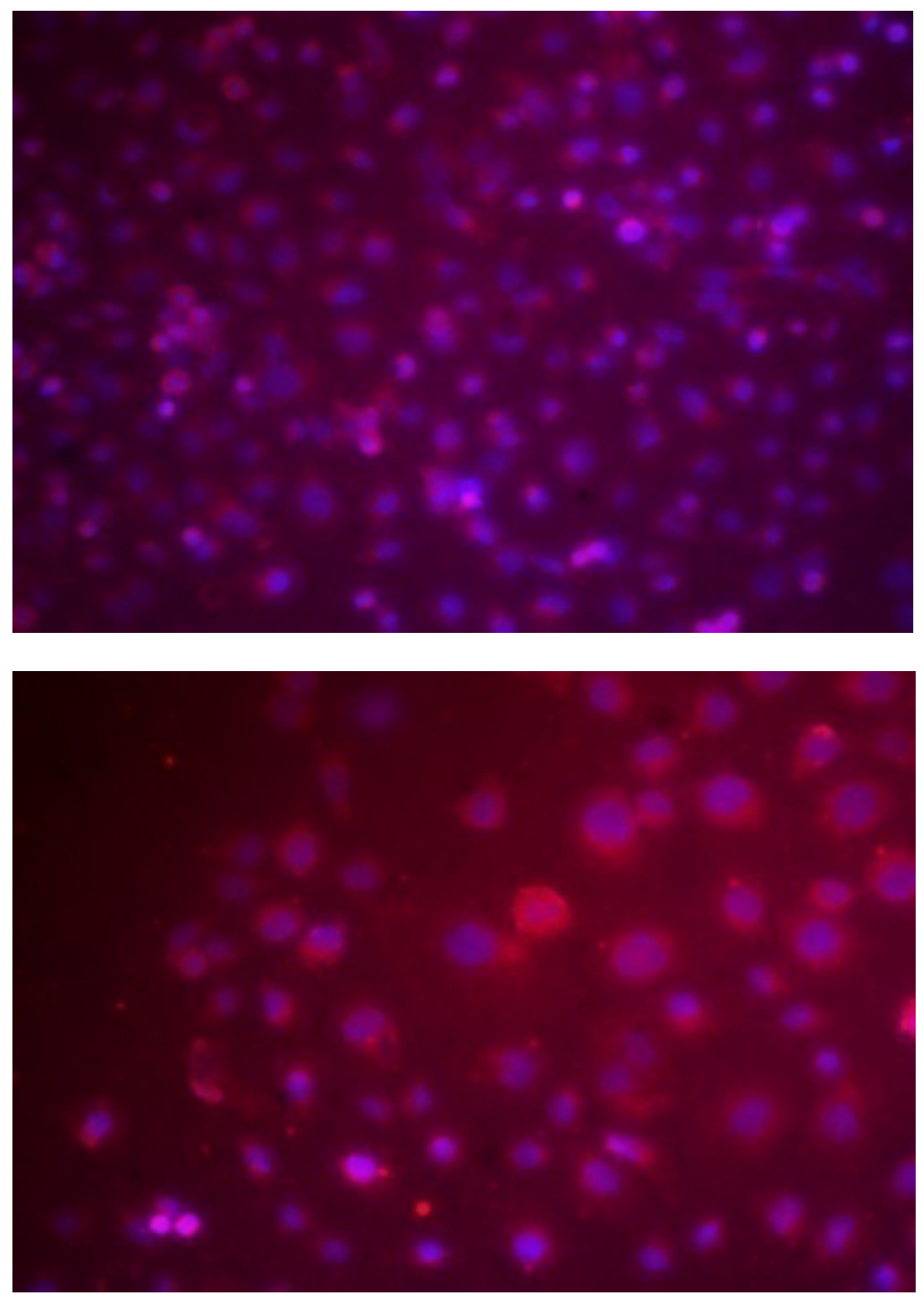

Figure 3.3. Fluorescence images of SKOV-3 cell culture on: (upper) petri dish, (middle) gelatin coated PDMS and (lower) uncoated PDMS.

\subsubsection{Direct measurement of VEGF from living cancer cells}

The time resolved SPR response upon $\mathrm{Ca}^{2+}$ ionophore injections is shown in Figure 3.4. Each arrow in the figure indicates an injection of $\mathrm{Ca}^{2+}$ ionophore. A $87 \pm 6 \mathrm{mDeg}(\mathrm{n}=4)$ SPR angle shift was observed after the first injection (data not shown for repeated 
measurements). However, the following two injections did not induce obvious angle shift. It seems that all intracellular VEGF was released during the first stimulation. According to our calibration curve for different concentrations of the VEGF samples, SKOV-3 cells released about $1 \mu \mathrm{g} / \mathrm{mL}$ VEGF during the stimulation. ${ }^{94}$

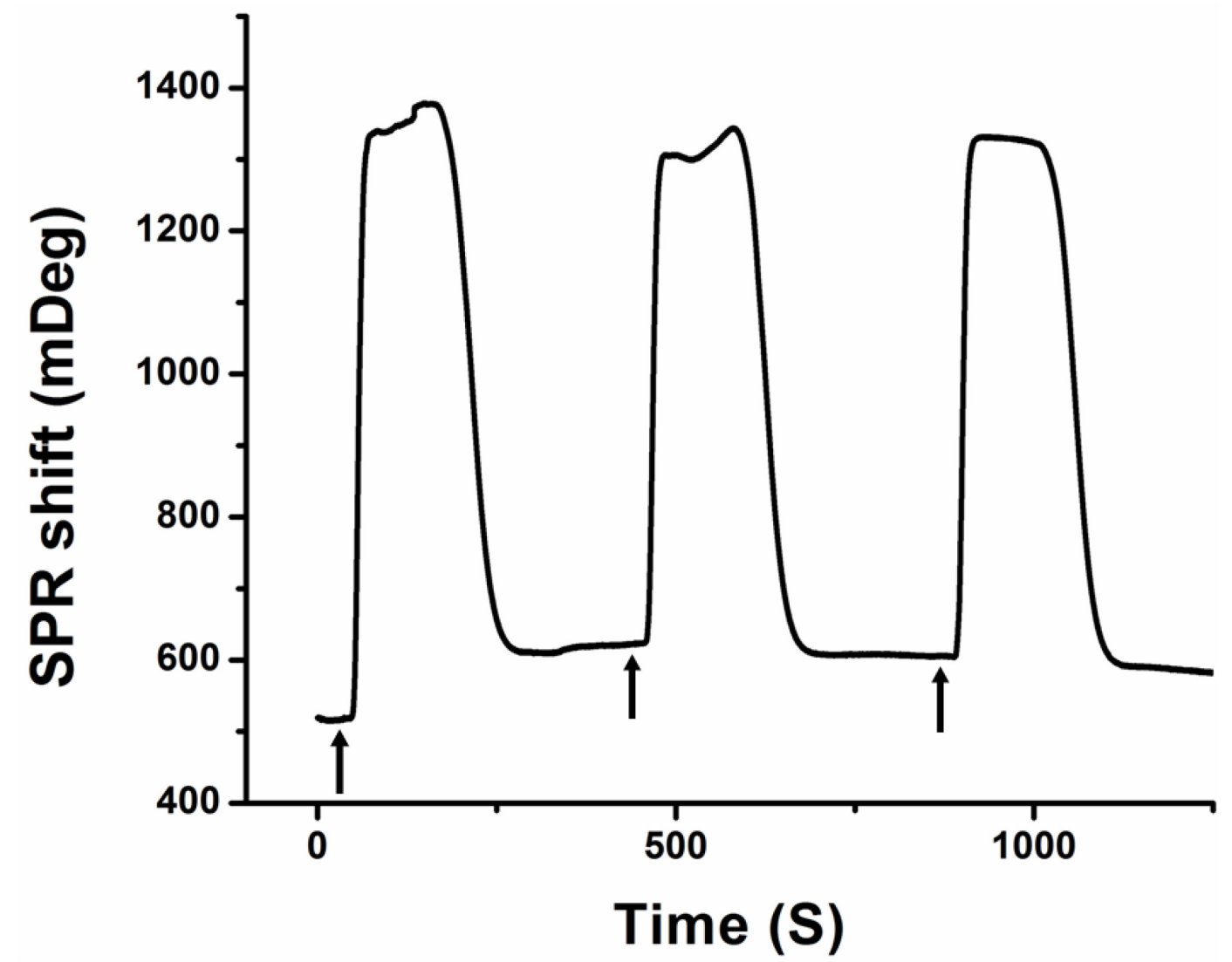

Figure 3.4. SPR sensor responses to living SKOV-3 cells secretion stimulated by injections of $500 \mu \mathrm{m} \mathrm{Ca}^{2+}$ ionophore (A23187). Each arrow indicates an injection of $\mathrm{Ca}^{2+}$ ionophore.

\subsubsection{Cell number study}

We also performed a cell number study to investigate the relationship between the amount of VEGF secretion and total cell number. Different numbers of cells (50000, $100000,200000)$ were seeded on three similar PDMS gaskets and cultured under the 
same conditions as previously mentioned. After $48 \mathrm{~h}$, the cell numbers reached 80000 , 160000 and 320000, respectively. SPR measurements were then performed 4 times for each of the 3 samples with different cell numbers. Figure 3.5 shows the SPR responses to induced VEGF secretion from different numbers of cells (data not shown for repeated measurements). SPR angle shift for each cell number group was determined by the average of 4 repeated measurements. From each averaged SPR angle shift, we can obtain the VEGF concentration secreted by the 3 different cell number groups. The SPR flow chamber volume is $2 \mathrm{~mL}$. Finally, we calculated the amount of VEGF released from each cell to be $0.0137 \pm 0.0012 \mathrm{pg}(\mathrm{n}=12)$ by averaging the VEGF singal cell release amount yielded for each of the 3 cell number groups. Due to the capacity limitation of our SPR flow chamber, the sample of 320000 cells had reached $100 \%$ confluency inside the chamber. Therefore, we were not able to further investigate the VEGF release amount from a larger group of SKOV-3 cells. However, this sensing platform has provided a strategy for accurate prediction of carcinoma cell number. 


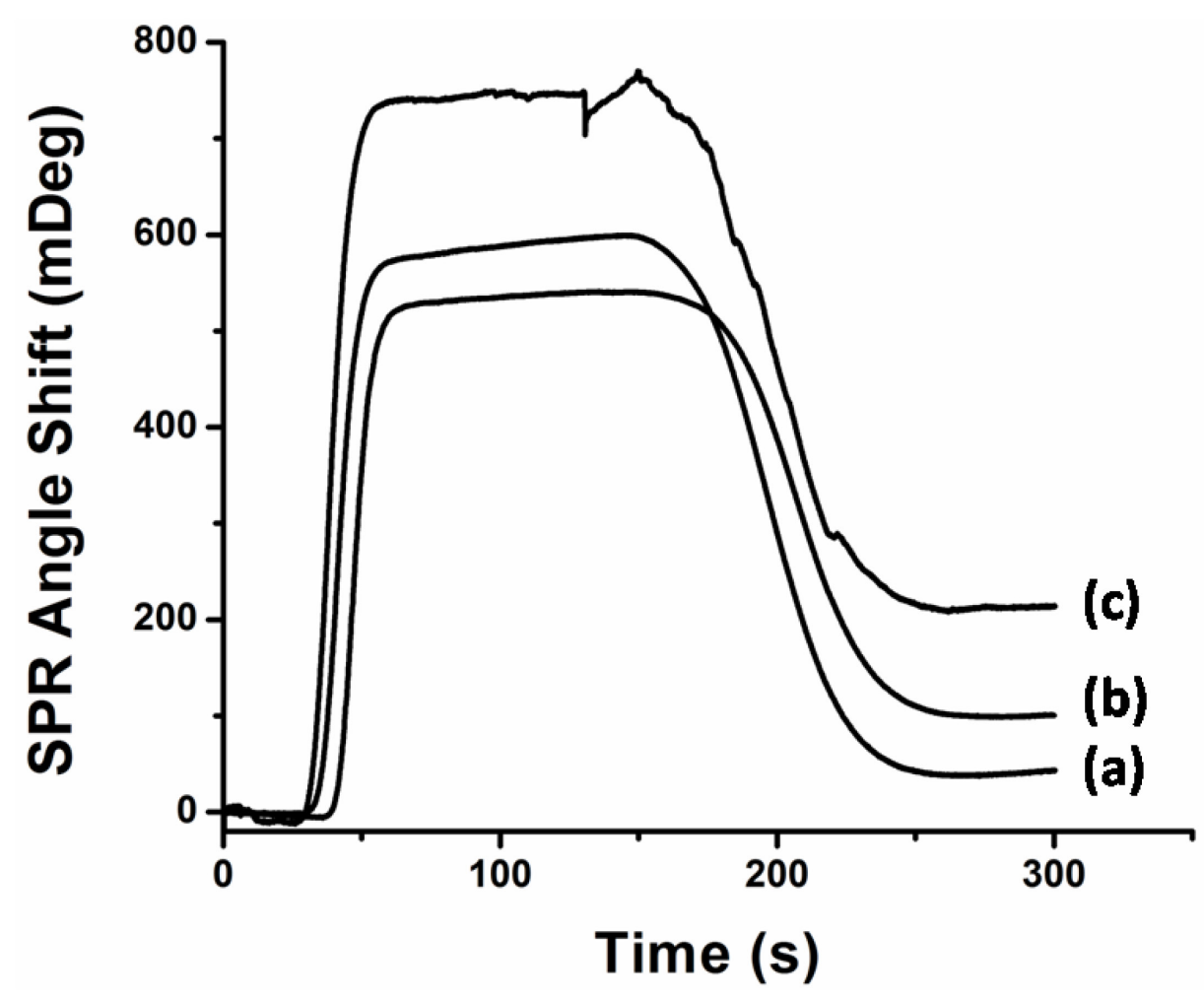

Figure 3.5. SPR sensor responses to stimulated VEGF secretion from groups of cells with different cell numbers. VEGF secretion from (a) 80000 cells induced $43.4 \pm 5.7 \mathrm{mDeg}(\mathrm{n}=4) \mathrm{SPR}$ angle shift; (b) 160000 cells induced $100.6 \pm 8.9 \mathrm{mDeg}(\mathrm{n}=4)$ SPR angle shift; (c) 320000 cells induced $214.2 \pm 15.1 \mathrm{mDeg}(\mathrm{n}=4)$ SPR angle shift.

\subsection{Conclusions}

In conclusion, this work demonstrates the new concept of a SPR biosensor for biomarker study. On the basis of integration of a mini cell culture system within the traditional SPR sensing platform, this biosensor is capable of direct measurement of VEGF biomarker secretion from living SKOV-3 carcinoma cells. Because the configuration of this SPR biosensor mimics the in vivo microenvironment of the VEGF signaling pathway, this platform possesses great potential on cellular signaling pathways study and antineoplastic drug development. By modifying the surface functionalization of the SPR assay, this 
biosensor might open up new horizons for detection and analysis of biomarker from living cells and tissue for different diseases. 


\section{Chapter 4}

Study of drug regulation on the VEGF binding kinetics with a living cell model 


\subsection{Introduction}

We have constructed an upgraded SPR biosensor with the ability of direct detection of disease biomarkers from living cell models in the previous chapter. This sensing strategy also possesses the potential of drug regulation study with living cell models. In this chapter, we will further investigate the feasibility of performing antineoplastic drug regulation studies on the cellular level by SPR biosensors.

For the past decade, cancer ranked the second leading cause of death in the United States. In 2012, a total of 1,638,910 new cancer cases and 577,190 deaths from cancer occurred in the United States alone. ${ }^{95}$ According to the defination of the American Cancer Society, cancer is the general name for a group of more than 100 diseases. Although there are many kinds of cancer, all cancers start because abnormal cells grow out of control. $^{96-99}$ Cancer types can be grouped into 5 main categories: (1) Carcinoma - cancer that begins in tissues or in the skin that cover internal organs; (2) Sarcoma - cancer that begins in connective or supportive tissue such as: bone, muscle, cartilage, fat, blood vessels, etc.; (3) Leukemia - cancer that starts in blood-forming tissue such as the bone marrow and causes large numbers of abnormal blood cells to be produced and enter the blood; (4) Lymphoma and myeloma - cancers that begin in the cells of the immune system; (5) Central nervous system cancers - cancers that begin in the tissues of the brain and spinal cord. ${ }^{100-102}$ Although some cancers, like leukemia, lymphoma and myeloma, rarely form tumors; in most cases, cancer cells form a tumor. A tumor larger than a millimeter will starve itself of oxygen and energy unless new blood vessels are built to 
provide supplies. ${ }^{103-104}$ A process known as angiogenesis is found to be involved in building new blood vessels for many types of cancer.

Angiogenesis is a complex process defined as the growth of new blood vessels from existing vessels. ${ }^{105-106}$ Normal embryogenesis and embryonic development occurs under normal physiological conditions in adults, whereas angiogenesis occurs during tissue repair, ovulation and endometrial regulation. ${ }^{107-108}$ Mediators of angiogenesis such as VEGF stimulate endothelial cells to secrete proteases and plasminogen activators. Cells will then migrate, proliferate and eventually differentiate to form a new lumen vessel. ${ }^{109}$ Many pathological conditions involve or mimic the angiogenic process, such as: atherosclerosis, rheumatoid arthritis, diabetes, heart disease and solid tumor growth. ${ }^{110}$ It is well established that tumor progression requires angiogenesis for blood and oxygen supply. Cancers switch on angiogenesis by breaking the balance between production of angiogenic stimulus and inhibitor factors. ${ }^{43,111-112}$ The angiogenesis process consists of several steps: (1) Angiogenic factor production; (2) Release of angiogenic factor; (3) Endothelial cell receptor binding and activation; (4) Endothelial cells proliferation; (5) Membrane formation and stabilization. ${ }^{113}$ VEGF is a very common angiogenic factor and VEGF receptor (VEGFR) is a very common endothelial cell receptor. VEGFVEGFR binding process is the key point of neovascularization. ${ }^{14-115}$ Targeting the endothelial cells receptor binding and activation process is a potential strategy for cancer repression. However, many questions about the VEGF signaling pathway remains not clearly understood. 
The VEGF family and its receptors include: (1) VEGF-A for endothelial cells migration and mitosis, creation of blood vessel; (2) VEGF-B specifically for myocardial tissue; (3) VEGF-C for lymphangiogenesis; (4) VEGF-D for the development of lymphatic vasculature surrounding lung bronchioles; (5) PlGF for vasculogenesis, also involved in cancer; and VEGFR-1, VEGFR-2, VEGFR-3 (Scheme 4.1). ${ }^{90}$ Among these members, VEGF-A and VEGFR-1 are most widely involved in different types of cancer. ${ }^{116}$

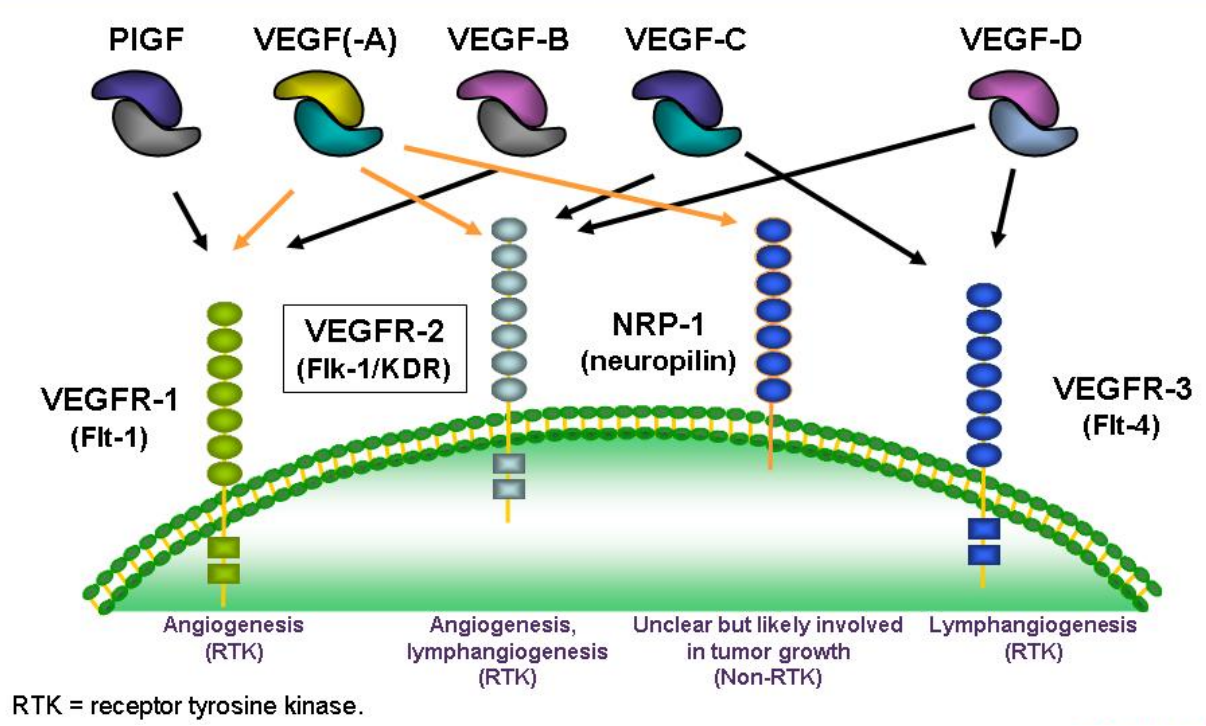

Scheme 4.1. Schematic diagram of the relationships between VEGF and VEGF receptors.

There are more than 100 antibodies currently approved by FDA or under development for cancer therapy by regulating VEGF-VEGFR binding interaction. ${ }^{117-118}$ One such approved antibody is bevacizumab, a humanized anti-VEGF monoclonal antibody generated by engineering the VEGF binding residues of a murine neutralizing antibody into the framework of the consensus human immunoglobulin $\left.\mathrm{G}_{1}\left(\operatorname{IgG}_{1}\right)\right)^{89}, 119$ Bevacizumab recognizes VEGFR-1 (flt-1) and VEGFR-2 (KDR) receptors and thus binds 
and neutralizes all biologically active forms of VEGF that interact with these receptors. ${ }^{120}$ The efficacy of bevacizumab against various cancer types has been demonstrated in several clinical studies (Table 4.1). ${ }^{121-125}$

\begin{tabular}{|l|l|l|}
\hline \multicolumn{1}{|c|}{ Indication } & \multicolumn{1}{|c|}{ Sample } & \multicolumn{1}{|c|}{ Conclusions } \\
Camber & 104 & Bevacizumab combined with chemotherapy was well \\
tolerated and may be effective in increasing response rates \\
and prolonging time to disease progression in patients with \\
metastatic colorectal cancer
\end{tabular}

Table 4.1. Summary of bevacizumab clinical studies.

Although there are numerous clinical studies and trials on the drug efficacy of bevacizumab on cancers, only a few fundamental studies on the interaction between this widely used drug and VEGF have been reported..$^{20,126-129}$ Kinetics study on VEGF- 
bevacizumab binding helps elucidate the fundamental mechanism of bevacizumab inhibition to angiogenesis. Tranditional techniques to measure the binding kinetic of VEGF and bevacizumab are Western blot and ELISA. ${ }^{130-131}$ These techniques can monitor cells only at a single time point and therefore do not allow for real-time monitoring. Another previous study has shown an in vitro real-time monitoring of VEGF-bevacizumab binding using SPR. ${ }^{20}$ However, the experimental condition was not similar to the in vivo VEGF signaling pathway since it was performed with the commercial VEGF solution.

In our previous studies, we have successfully demonstrated real-time monitoring VEGF expression from living human ovarian carcinoma cells using SPR. ${ }^{132}$ By intergrating a mini cell culture system into the SPR flow system, we were able to maintain live cell culture on the ceiling of the SPR flow chamber in order to realize VEGF measurements from living cells. In this study, we have measured and compared the binding affinity of VEGF to VEGFR and VEGF to bevacizumab. Finally, we employed this living cell sensing platform to mimic the in vivo condition of the VEGF signaling pathway. Competitive binding to VEGF between VEGFR and bevacizumab was monitored in realtime. The results have shown significant blockage of VEGF-VEGFR binding by bevacizumab. 


\subsection{Experimental}

\subsubsection{Materials}

Bevacizumab (200 mg) were friendly donated by Genentech, Inc. (San Francisco, CA) VEGF, VEGFR, KREB's buffer and Penicillin were obtained from Sigma-Aldrich (St. Louis, MO). SKOV-3 cells, McCoy's 5A medium, and fetal bovine serum were purchased from American Type Culture Collection (Manassas, Virginia). 6-well tissue culture plates, d-poly coverslips, and formalin were purchased from Fisher Scientific. MUA was purchased from Asemblon (Redmond, WA). Calcium Ionophore (A23187) was purchased from Acros Organic (New Jersey). EDC and NHS were obtained from Alfa Aesar (WardHill, MA). Protein G was purchased from Fisher Scientific (Waltham, MA). All reagents and solvents were used as received unless otherwise stated.

\subsubsection{Instrumentation}

A BI-2000 SPR instrument purchased from Biosensing Instrument (Tempe, AZ) was used for data collection. A $670 \mathrm{~nm}$ wave length laser with a $72.2^{\circ}$ incident angle was employed as the light source of the SPR instrument. The gold SPR chip $(20 \mathrm{~mm} \times 20 \mathrm{~mm} \times 1 \mathrm{~mm})$ is a BK7 glass slide coated with a $45 \mathrm{~nm}$ layer of gold over a $5 \mathrm{~nm}$ layer of chromium. A biocompatible PDMS microfluidic injection chamber gasket with a $5 \mathrm{~mm} \times 1.7 \mathrm{~mm} \times 125 \mu \mathrm{m}$ channel was clamped on top of the gold face of the chip. A dual syringe pump was attached to the injection chamber allowing both sample and reference buffer flow through the sensing surface. An Olympus IX81 fluorescence microscopy (Japan) with a 20x objective was employed for cell fluorescent imaging. A CCD camera was used to capture the signals and the images were software-merged with pseudo color. 
4.2.3. SPR flow chamber surface pre-treatment and human cell culture SPR flow chamber gasket was detached from the SPR flow cell. Several drops of $0.1 \%$ w/v gelatin solution made by boiling distilled water were applied onto the PDMS gasket to cover the whole surface. The gasket was then dried for $12 \mathrm{~h}$ in a biological fume hood to prevent contamination. ${ }^{133}$ A cell culture petri dish and a uncoated PDMS gasket were also prepared as positive and negative control substrates, respectively.

SKOV-3 cells were cultured in McCoy's 5A medium added with $1 \%$ penicillin and $10 \%$ fetal bovine serum, and kept in a $37^{\circ} \mathrm{C}$ cell incubator with a humidified atmosphere of $5 \% \mathrm{CO}_{2}$ and $95 \%$ air. 200,000 cells were seeded on the gelatin coated SPR flow chamber gasket and kept in a 6-well tissue culture plates with cell culture media for $48 \mathrm{~h}$ before each experiment. The same cell culture was repeated on an uncoated PDMS gasket and a tissue culture plate as control experiments.

\subsubsection{Cell stain and fluorescent imaging}

We diluted $200 \mu \mathrm{M}$ MitoTracker Red CMXRos dye and the $1.0 \mathrm{mM}$ Hoechst 33342 dye stock solutions into cell medium with serum. The concentration for MitoTracker Red CMXRos dye is $10-50 \mathrm{nM}$; The concentration for Hoechst 33342 dye is $1.0 \mu \mathrm{g} / \mathrm{mL}$. Both dyes may be combined in a single staining solution. A sufficient amount of labeling solution was then applied to cover cells adhering to substrates followed by incubation for 15 minutes at $37^{\circ} \mathrm{C}$. After that, the labeling solution was removed and cells were washed twice in the cell medium. Labeled cells will then be fixed with $4 \%$ formaldehyde for 15 
minutes at $37^{\circ} \mathrm{C}$, followed by washes in buffer and staining with any additional counterstains.

The substrates were then removed and mounted on glass microslides with antifade reagent/mounting medium mixture. Then, the specimens were observed by fluorescence microscopy (Olympus IX81, Japan) with a 20x objective. The fluorescence was imaged at Hoechst channel (nucleus stain dye, ex/em: 358/461 nm), and mitotracker red channel (ex/em: 570/590 nm). A CCD camera was used to capture the signals and the images were software-merged with pseudo color. The fluorescence microscope settings were kept the same throughout the experiment with the exception of the exposure time. The images were recorded at the same exposure time for Hoechst and MitoTracker Red.

\subsubsection{Preparation of SPR gold sensing surface}

A similar method reported in our previous study was used for surface modification of the SPR gold chip. ${ }^{132}$ Briefly, SPR gold chip will be emerged in 1mM MUA (ethanol) solution for $12 \mathrm{~h}$ at $4{ }^{\circ} \mathrm{C}$ to form a layer of carboxylic groups on the gold surface. The gold chip will then be washed with copious ethanol and mounted on the SPR system. Carboxylic groups were then activated with an injection of EDC (75mM) / NHS (15mM) solution. All injections to the SPR microfluidic system in this study were performed with a $20 \mu \mathrm{L} / \mathrm{min}$ rate. Surface modification and immobilization experiments were performed in PBS as the flowing buffer, and each solution was also prepared in PBS $(\mathrm{pH}=7.4)$ unless otherwise specified. 


\subsubsection{Comparison of VEGF-VEGFR and VEGF-bevacizumab binding kinetics}

In order to demonstrate the drug efficacy of bevacizumab, binding affinity of VEGFVEGFR and VEGF-bevacizumab interactions was measured and compared. Following the surface modification of the SPR gold chip, an activated SPR chip was immobilized by injecting $50 \mu \mathrm{g} / \mathrm{mL}$ protein $\mathrm{G}$ solution to capture the $\mathrm{Fc}$ portions of the bevacizumab antibody in order to assure proper antibody orientation. $30 \mu \mathrm{g} / \mathrm{mL}$ bevacizumab was then bounded on top of the protein G layer. Another SPR chip was immobilized by direct injection of $30 \mu \mathrm{g} / \mathrm{mL}$ VEGFR onto the activated surface. $2 \mu \mathrm{g} / \mathrm{mL}$ VEGF was injected to the flow chamber after surface functionalization. SPR sensorgram was collected by Biosensing Instrument SPR Control Program running on a PC.

\subsubsection{Drug regulation study on VEGF-VEGFR binding}

A biomimic system was constructed to evaluate the bevacizumab drug regulation on the VEGF signaling pathway. VEGFR was immobilized on the SPR gold sensing surface by aforementioned method. The flowing PBS buffer was paused once VEGFR was immobilizaed. The PDMS flow chamber gasket used for surface immobilization (no cells) was removed.

The PDMS flow chamber gasket with SKOV-3 cell culture was then removed from cell culture media and throughly rinsed with Kreb's buffer to remove cell culture media and unattached cells. Following this, the PDMS gasket with cells was mounted on the SPR flow chamber, and SPR flowing buffer was changed from PBS $(\mathrm{pH}=7.4)$ to Kreb's buffer $(\mathrm{pH}=7.4)$ in order to maintain cell viability during experiments. After resuming 
the buffer flow, $500 \mu \mathrm{m} \mathrm{Ca}^{2+}$ ionophore (A23187) was injected to induce rapid exocytosis of VEGF from the SKOV-3 cells. We added $30 \mu \mathrm{g} / \mathrm{mL}$ bevacizumab to the flowing buffer to capture the VEGF secretion. Therefore, we have built an in vitro system to mimic the VEGF-VEGFR release, transportation, binding processes to monitor the bevacizumab drug regulation in real-time (Scheme 4.2).

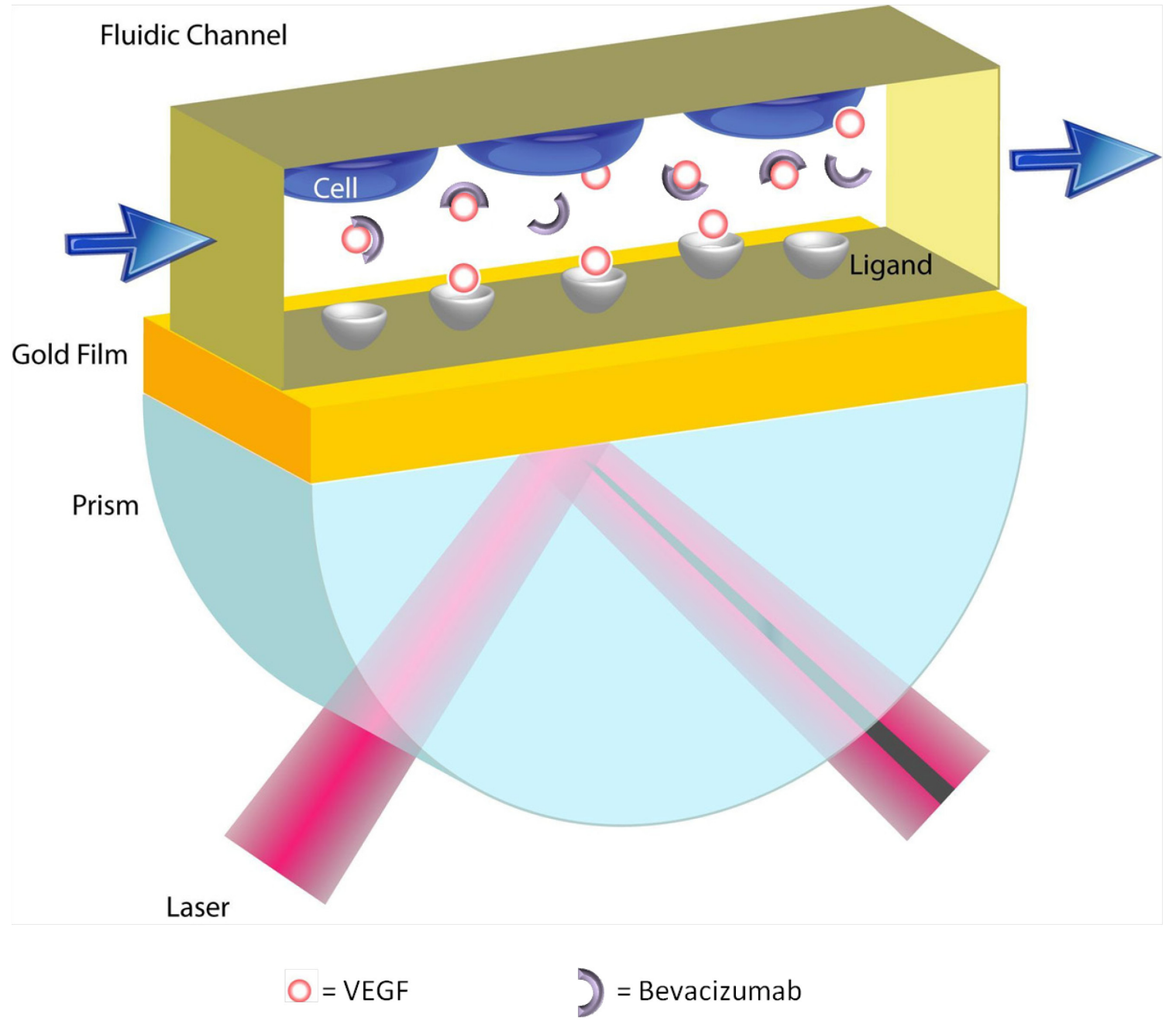

Scheme 4.2. Schematic illustration of the biomimic system for bevacizumab drug regulation study. 


\subsection{Results and discussion}

\subsubsection{Optimization and calibration for surface concentration of bevacizumab}

In order to determine the optimal dose of bevacizumab for this study, we investigated the SPR response to different concentrations immobilized on the gold sensing surface. Initially, $50 \mu \mathrm{g} / \mathrm{mL}$ protein $\mathrm{G}$ solution was injected to the activated SPR chip followed by injection of a certain concentration of bevacizumab $(5 \mu \mathrm{g} / \mathrm{mL}, 10 \mu \mathrm{g} / \mathrm{mL}, 30 \mu \mathrm{g} / \mathrm{mL}$, $50 \mu \mathrm{g} / \mathrm{mL}, 70 \mu \mathrm{g} / \mathrm{mL})$. Three gold chips were prepared for each concentration. $3 \mu \mathrm{g} / \mathrm{mL}$ VEGF was then injected to pass through the sensing surface. The SPR measurement was repeated 3 times for each bevacizumab concentration. Figure 4.1 shows the results from one set of measurements. The resulting SPR response for each bevacizumab concentration was plotted against time $(70 \mu \mathrm{g} / \mathrm{mL}$ data not shown). It is evident that the amount of VEGF bound to the $50 \mu \mathrm{g} / \mathrm{mL}$ bevacizumab sensing surface did not show a significant difference than the amount of VEGF bound to the $30 \mu \mathrm{g} / \mathrm{mL}$ bevacizumab sensing surface (Figure 4.2). This observation can be attributed to two possible reasons: (1) $30 \mu \mathrm{g} / \mathrm{mL}$ is the saturation concentration of bevacizumab on the surface or (2) $3 \mu \mathrm{g} / \mathrm{mL}$ VEGF injection can be completely captured by $30 \mu \mathrm{g} / \mathrm{mL}$ surface bound bevacizumab. Our previous study showed that even SKOV-3 cells reached $100 \%$ confluency in the SPR flow chamber, the VEGF release did not reach $3 \mu \mathrm{g} / \mathrm{mL}$. As a result, we employed $30 \mu \mathrm{g} / \mathrm{mL}$ as the maximum concentration of bevacizumab for further binding studies. Linear responses of bevacizumab-VEGF binding are obtained between $5 \mu \mathrm{g} / \mathrm{mL}$ and $30 \mu \mathrm{g} / \mathrm{mL}$ of surface bound bevacizumab concentrations. 


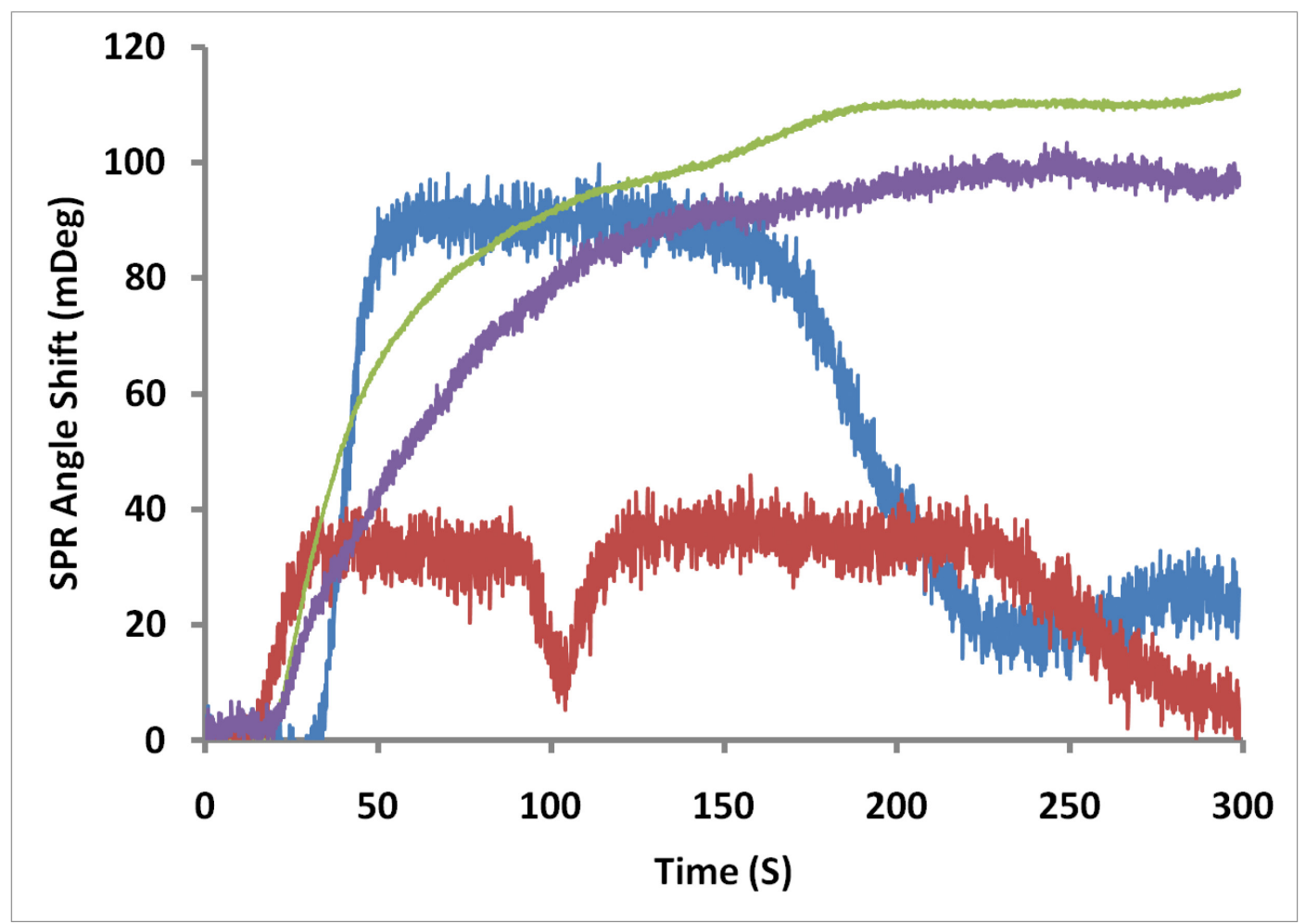

Figure 4.1. SPR response to $3 \mu \mathrm{g} / \mathrm{mL}$ VEGF for each surface bound bevacizumab concentration: $5 \mu \mathrm{g} / \mathrm{mL}$ (red), $10 \mu \mathrm{g} / \mathrm{mL}$ (blue), $30 \mu \mathrm{g} / \mathrm{mL}$ (purple), $50 \mu \mathrm{g} / \mathrm{mL}$ (green). 


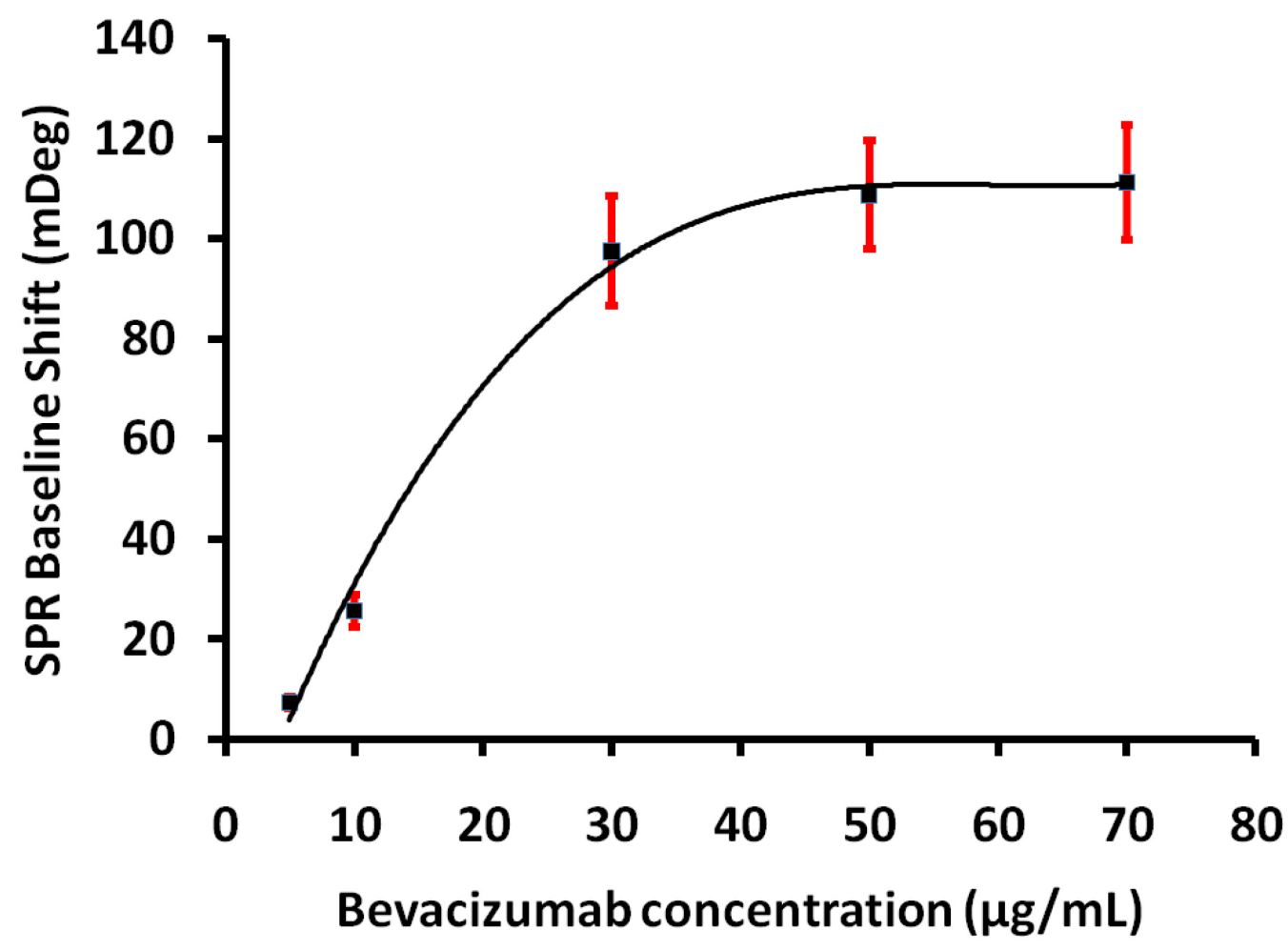

Figure 4.2. Calibration curve: the linear relationship between SPR baseline shift and the surface concentration of bevacizumab $(n=3)$.

\subsubsection{Specificity study of VEGF-bevacizumab binding}

Bevacizumab is a humanized anti-VEGF monoclonal antibody generated by engineering the VEGF binding residues of a murine neutralizing antibody into the framework of the human IgG. Therefore, it is only expected to bind with VEGF specifically. ${ }^{134}$ Any nonspecific binding to other biomolecules is going to affect the efficacy of this drug. We have already demonstrated the affinity and reproducibility of VEGF-bevacizumab binding in the previous experiment. In this experiment, we further investigated the specificity of the VEGF-bevacizumab interaction. VEGF-VEGFR binding and activation is the most important step of the angiogenesis process, which is also the target of most 
angiogenesis regulation therapy strategies, including bevacizumab. Thus it is crucial to investigate the interaction between bevacizumab and VEGFR. Similarly, a bevacizumab layer was formed on the activated SPR gold surface by injecting $10 \mu \mathrm{g} / \mathrm{mL}$ bevacizumab. Following this, $3 \mu \mathrm{g} / \mathrm{mL}$ VEGF and VEGFR were injected and passed the two bevacizumab covered gold chips, respectively. As shown in Figure 4.3A, a 25.1mDeg baseline shift was observed on the SPR sensorgram due to the VEGF-bevacizumab binding. However, no significant baseline shift was detected for VEGFR-bevacizumab interaction (Figure 4.3B). The results indicate that bevacizumab regulates the angiogenesis process by blocking the active binding site on VEGF. The specificity study was then repeated for 3 times. Similar results were observed. 


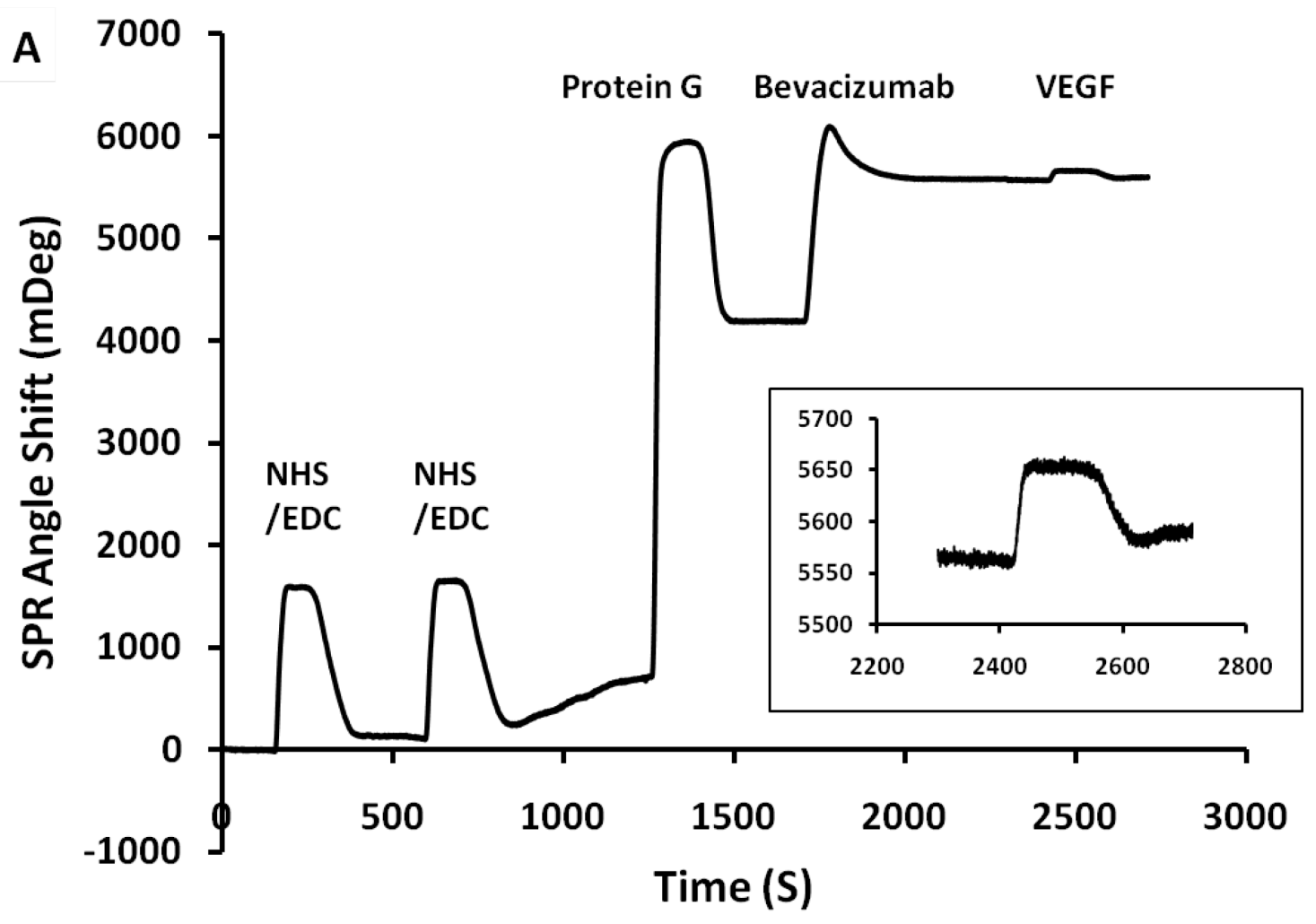

Figure 4.3. (A) SPR sensorgram of surface bound bevacizumab interacting with VEGF. Inset: enlarged sensorgram of the response upon VEGF binding. 


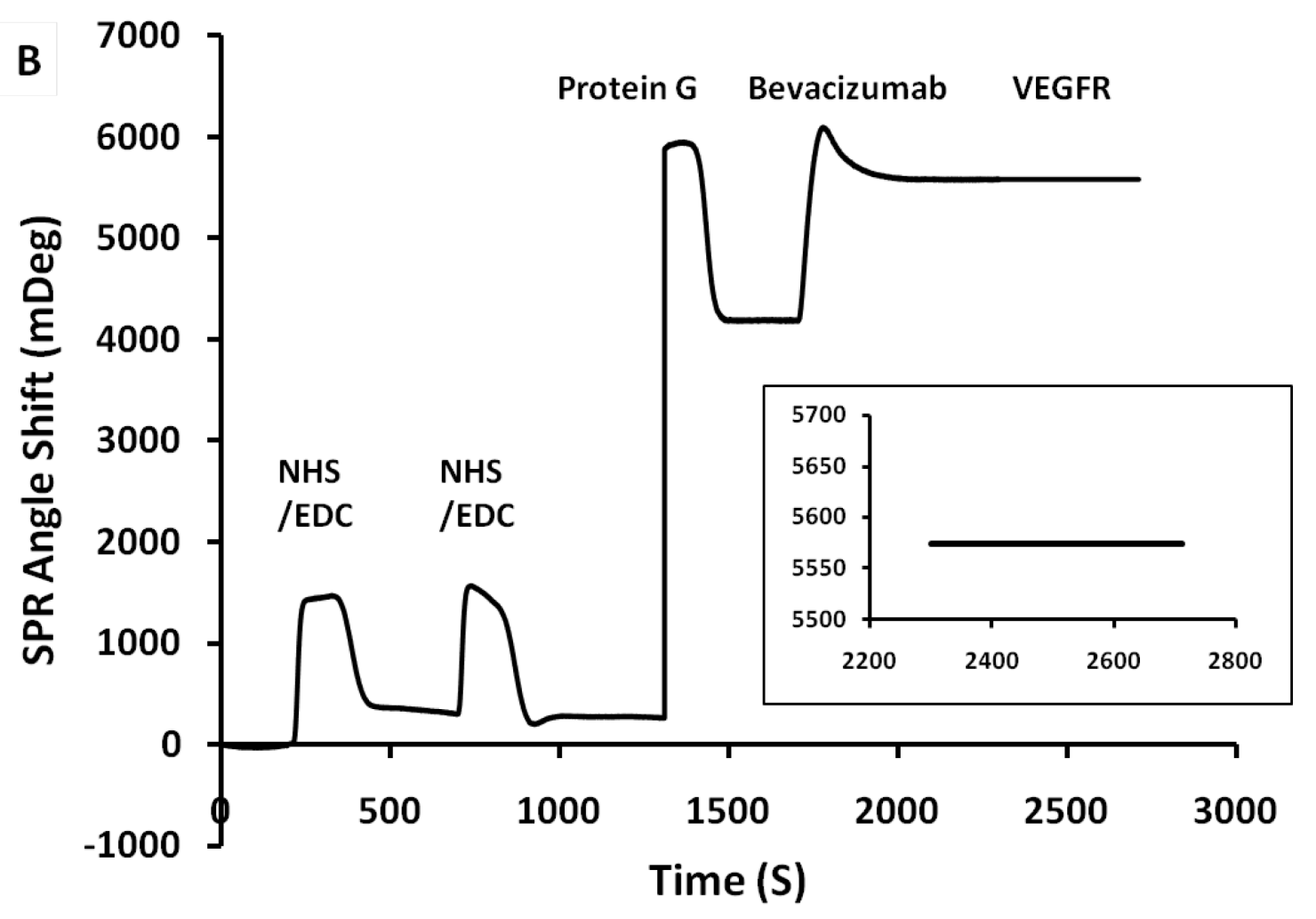

Figure 4.3. (B) SPR sensorgram of surface bound bevacizumab interacting with VEGFR. Inset: enlarged sensorgram of the response upon VEGFR binding.

\subsubsection{Study of SKOV-3 cell viability in the SPR flow chamber}

For the purpose of constructing the VEGF signaling pathway biomimic system for the drug efficacy study, we first demonstrated cell viability in the SPR flow chamber. Fluorescent imaging was performed using the aforementioned method in order to examine cell confluency on each type of substrate. As shown in Figure 4.4, the cell confluency on tissue culture plates and gelatin coated gaskets both reached $100 \%$, whereas the cell confluency on uncoated gaskets only reached $60 \%$. Gelatin coated gaskets showed significant enhancement of cell attachment compared to uncoated gaskets. Therefore, it is a suitable substrate for living cell experiments in the SPR flow 
chamber.

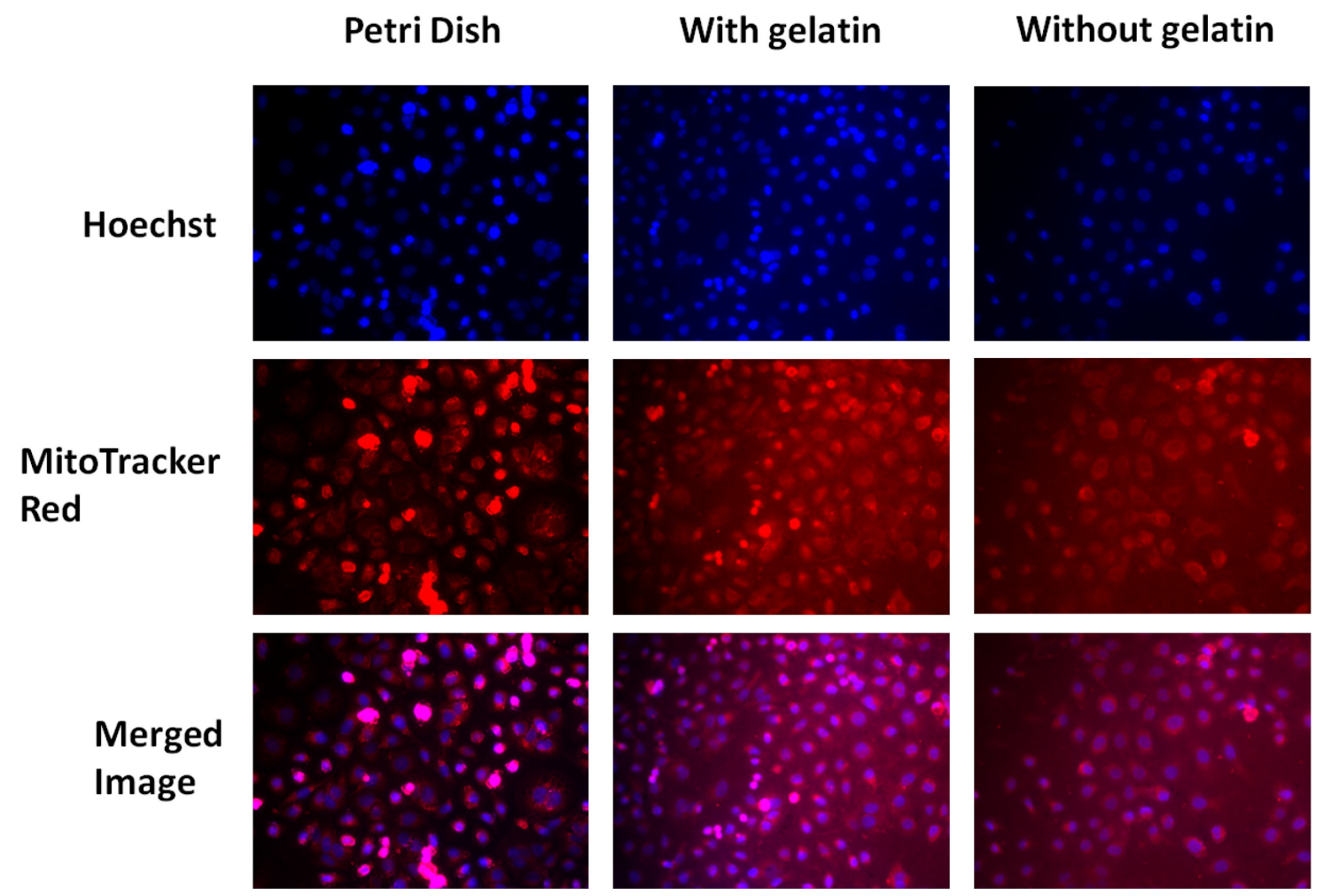

Figure 4.4. Fluorescent images of SKOV-3 cells at Hoechst channel, MitoTracker Red channel and merged images on different type of substrates: petri dishs (exposure time 400ms), gelatin coated gaskets (exposure time 200ms) and uncoated gaskets (exposure time 200ms).

\subsubsection{Binding kinetics study of VEGF-VEGFR and VEGF-bevacizumab interactions}

We have previously shown the binding affinity of VEGF-bevacizumab interaction.

Herein, binding kinetics of the VEGF-bevacizumab interaction was compared to that of the VEGF-VEGFR interaction using the biomimic sensing platform.

Initially, bevacizumab was immobilized on the SPR gold sensing surface using the aforementioned protocol. Following this, $2 \mu \mathrm{g} / \mathrm{mL}$ VEGF solution was injected to pass the sensing surface. A similar experiment was then performed with VEGFR immobilized 
on the SPR gold sensing surface. Both experiments were repeated 4 times for reproducibility test. The SPR sensorgram of the responses on VEGF binding to bevacizumab (blue) and VEGFR (red) is shown in Figure 4.5 (data not shown for repeated measurements).

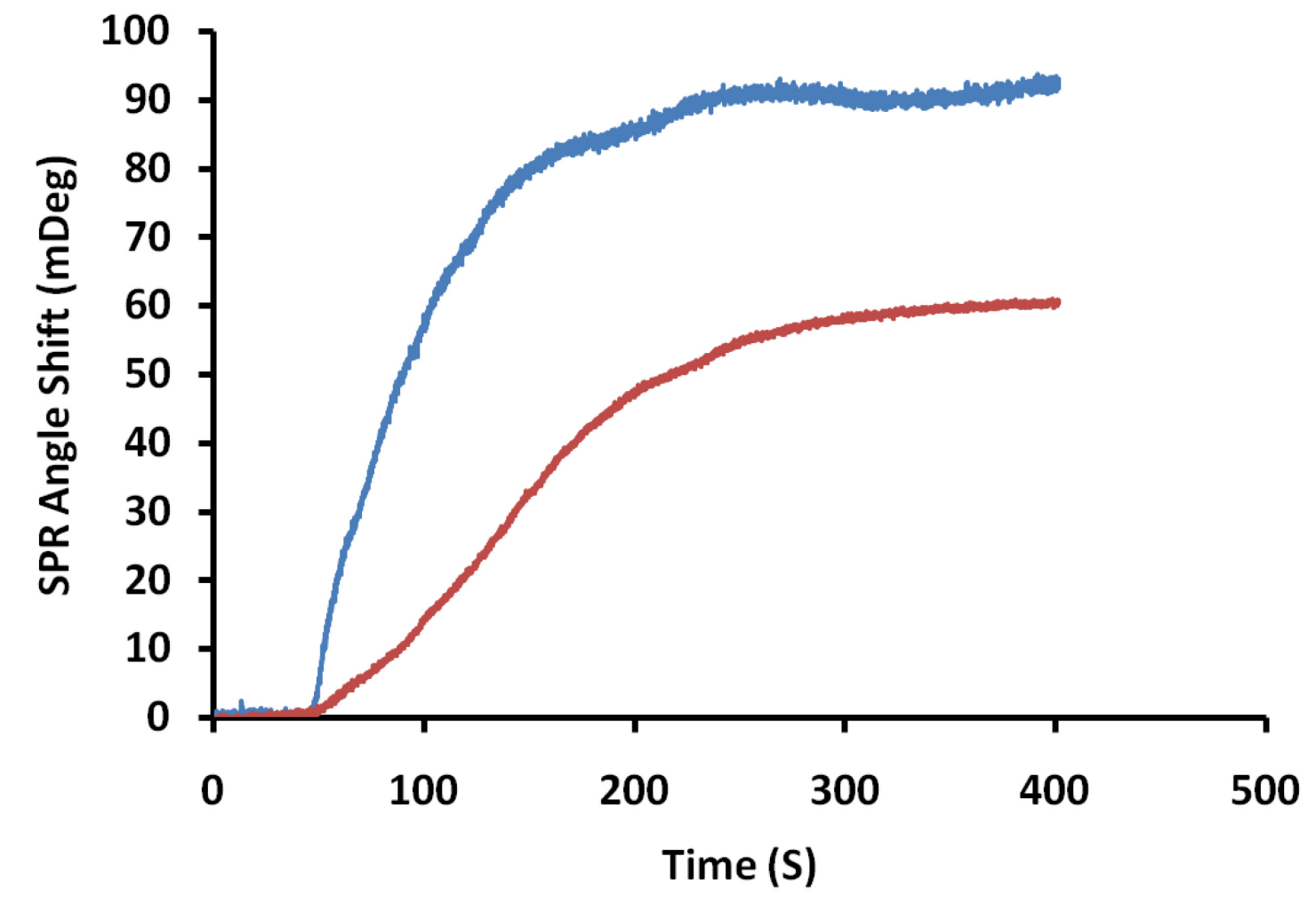

Figure 4.5. SPR sensorgram of VEGF binding response to bevacizumab (blue line) and VEGFR (red line).

A modified equation of a previous reported pseudo-first-order kinetic equation ${ }^{135}$ was used to determine the association rate constants:

where $\mathrm{R}$ is the SPR signal at time $\mathrm{t}, \mathrm{k}_{\mathrm{a}}$ is the association rate constant which indicates the binding affinity between two reactants, [VEGF] and [ligand] are the concentration of 
VEGF and the surface bound binding ligand (bevacizumab or VEGFR), respectively. The maximum value of $(\mathrm{dR} / \mathrm{dt})$ for each sample was determined by calculating the maximum slope of the association curve using Matlab. The weight concentration of VEGF was converted to molar concentration $(47.6 \mathrm{nM})$. The concentration of the surface bound bevacizumab is $30 \mu \mathrm{g} / \mathrm{mL}(201.3 \mathrm{nM})$. As shown in Figure 4.5, the maximum slope of the VEGF-bevacizumab binding curve was calculated to be $0.91 \pm 0.04(n=4)$. Thus the $\mathrm{k}_{\mathrm{a}}$ for bevacizumab is $9.5 \pm 0.4 \times 10^{13} \mathrm{M}^{-2}(\mathrm{n}=4)$. Similarly, the concentration of the surface bound VEGFR is $30 \mu \mathrm{g} / \mathrm{mL}(166.7 \mathrm{nM})$, the maximum slope of the VEGF-VEGFR binding curve was calculated to be $0.43 \pm 0.01(\mathrm{n}=4)$. Therefore the $\mathrm{k}_{\mathrm{a}}$ for VEGFR is $5.4 \pm 0.2 \times 10^{13} \mathrm{M}^{-2}(\mathrm{n}=4)$. We also obtained the binding affinity information for both interactions from Figure 4.5 by measuring the SPR baseline shift upon VEGF binding. VEGF-bevacizumab binding induced a $92.7 \pm 4 \mathrm{mDeg}(\mathrm{n}=4)$ baseline shift, whereas VEGF-VEGFR binding induced a $60.2 \pm 3 \mathrm{mDeg}(\mathrm{n}=4)$ baseline shift. All aforementioned calculations were obtained by the average of 4 repeated measurements. These results indicate that bevacizumab captures VEGF with a higher rate and affinity compared to VEGFR. $^{20}$

\subsubsection{Bevacizumab drug regulation study on VEGF-VEGFR interaction}

According to the aforementioned protocol, the drug efficacy of bevacizumab was evaluated using the VEGF signaling pathway biomimic system, as shown in Figure 4.6. Briefly, VEGFR was immobilized on the SPR gold sensing surface. PDMS flow chamber gasket with SKOV-3 cell culture was mounted on top of the sensing surface. We employed pure Kreb's buffer and Kreb's buffer with $30 \mu \mathrm{g} / \mathrm{mL}$ bevacizumab as the 
SPR flowing buffers for the control experiment and the drug regulation experiment, respectively. $\mathrm{Ca}^{2+}$ ionophore (A23187) was used to stimulate rapid exocytosis of VEGF from the SKOV-3 cells. Both the control experiment and the drug regulation experiment were repeated 4 times under similar condition. In Figure 4.6, the red line depicts the SPR response of VEGF exocytosis binding to VEGFR on the sensing surface (control experiment). The peak between $\sim 50 \mathrm{~s}$ and $\sim 250 \mathrm{~s}$ can be attributed to the refractive index change induced by the $\mathrm{Ca}^{2+}$ ionophore (A23187) passing through the sensing surface. Following the plateau, the baseline is stablized at $103.7 \pm 8.4 \mathrm{mDeg}(\mathrm{n}=4)$. This SPR baseline shift indicates that VEGF released from SKOV-3 cells has bound to the VEGFR. The blue line depicts the SPR response of VEGF exocytosis binding to surface VEGFR under the regulation of bevacizumab (drug regulation experiment). By comparing this data to the control experiment, the SPR base line has dropped by $82.5 \pm 9.6 \mathrm{mDeg}(\mathrm{n}=4)$ after the $\mathrm{Ca}^{2+}$ ionophore (A23187) stimulation. We anticipate this sudden drop of baseline is due to the surface refractive index decrease caused by the fast binding between VEGF and bevacizumab in the flowing buffer. However, further experiments are needed to confirm this assumption. Following the plateau, the baseline is slowly increasing towards the original position, since the VEGF exocytosis was being carried away from the sensing surface. No significant baseline increase was observed in this experiment, which indicates that the VEGF-VEGFR pathway was successfully blocked by bevacizumab in the flow chamber. The amount of VEGF bound to VEGFR was significantly decreased under the regulation of bevacizumab. ${ }^{136}$ 


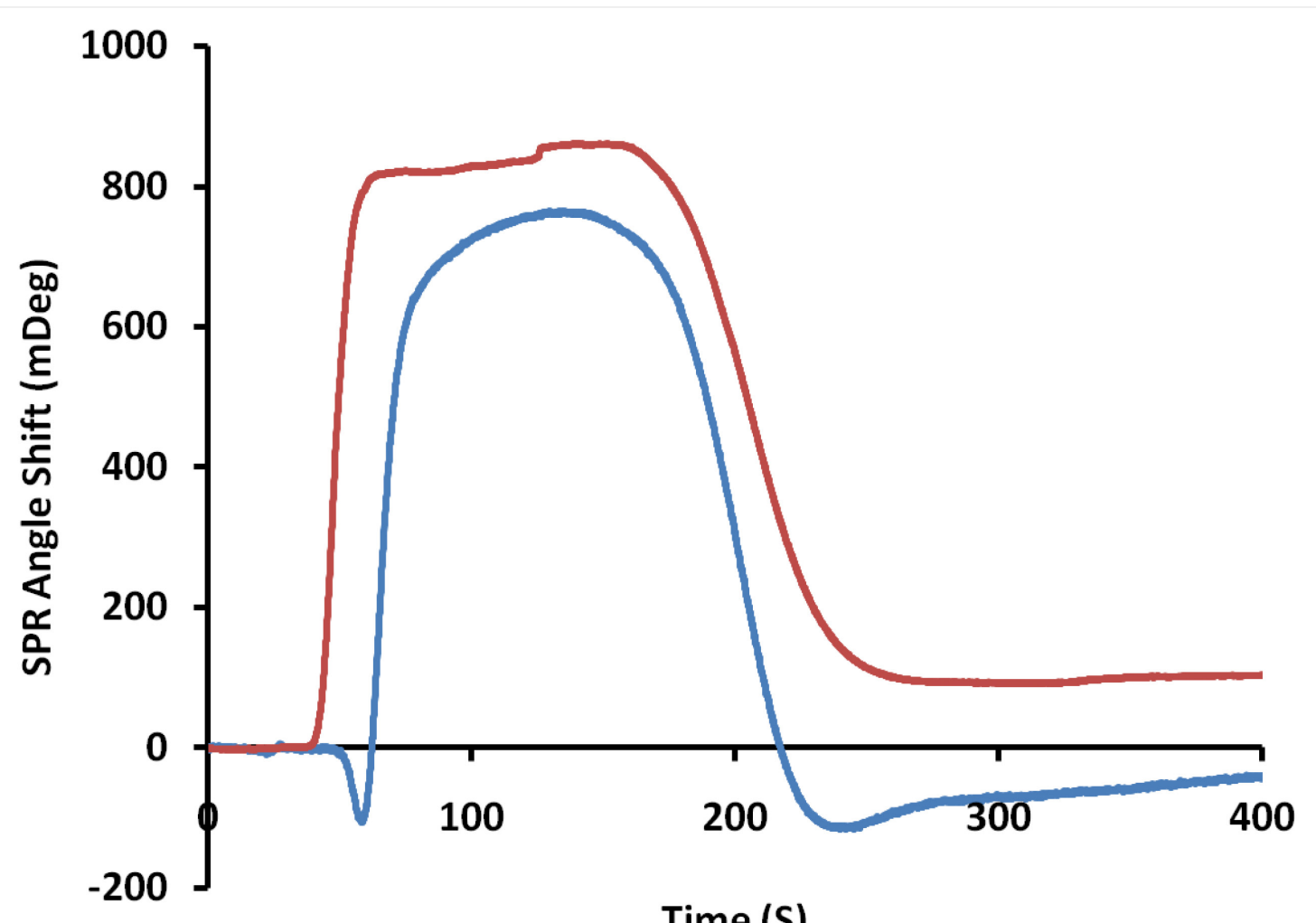

Time (S)

Figure 4.6. SPR response of VEGF (SKOV-3 cells released) - VEGFR interaction (red line) and VEGF (SKOV-3 cells released) - VEGFR interaction under bevacizumab regulation (blue line) in the biomimic system.

\subsection{Conclusions}

In this study, we have constructed a biomimic system for the VEGF-VEGFR signaling pathway in SPR and investigated its potential application on antineoplastic drugs development. To the best of our knowledge, this is the first report showing direct and real-time measurements of drug effect to the VEGF-VEGFR signaling pathway on living carcinoma cells. SKOV-3 cells and the bevacizumab antibody were used as the cell model and the drug model for the evaluation of the strategy. SPR exhibits excellent sensitivity and linear dynamic range towards VEGF and bevacizumab interactions. SKOV-3 cells were incubated in the modified SPR flow chamber for the drug efficacy 
study. VEGF-bevacizumab binding showed a higher association rate constant and binding affinity than VEGF-VEGFR binding. The bevacizumab drug regulation study reveals successful blockage of VEGF-VEGFR binding and activation process. We have outlined a highly sensitive and simple strategy for the direct and real-time measurements of bevacizumab drug efficacy to the VEGF-VEGFR signaling pathway on living SKOV3 cells. It is hopeful that a similar strategy may be employed to develop and evaluate other type of medicine. 
Chapter 5

Conclusions and Future Work 


\subsection{Conclusions}

This dissertation demonstrates real-time, direct and sensitive LSPR based biosensors for biomolecular binding kinetics measurements. We have achieved a LSPR sensing platform for the detection of small heavy metal molecule bindings. The performance of this sensor was evaluated on the study of arsenic detoxification pathway of $E$. coli. We also successfully incorporated a mini cell culture system into the flow chamber of the SPR based biosensors. This novel feature enables the in vitro detection of biomarkers from living cells. Following this, the SPR based biosensors with the capability of biomarker measurements from living cells were employed on the drug regulation study of the VEGF signaling pathway in cancer cells.

Chapter 1 introduces the objectives, the background and literature review on the dissertation topic. Three specific aims of this dissertation are also presented.

Chapter 2 addresses the specific aim 1. It deals with the development of highly sensitive LSPR sensor and its application on detection of small heavy metal molecule binding. A self-assembled 3D hydrogel-nanoparticle composite integrated LSPR sensor is reported. The novel assembled substrate was developed by means of a surface mediated radical copolymerization process to obtain a highly sensitive hydrogel-based thin film that possesses specific binding sites for target analytes. Initially, amino group modified AuNPs were covalently linked to acrylic acid monomer. NIPAAm and AuNPs linked AAc monomers were randomly co-polymerized by the "grafting from" method in the presence of initiator and crosslinker onto the sensing surface. Surface charecterization 
techniques were utilized to evaluate the thickness and composition of the hydrogelnanoparticle film. The sensing platform was employed to study the binding kinetics and conformational changes of the ArsA ATPase as a consequence of binding trivalent arsenicals under a variety of conditions. ArsA, the catalytic subunit of the ArsAB As(III) translocating ATPase, is one of the five proteins encoded by the ars operon of plasmid R773 in cells of E. coli, that confers resistance to trivalent and pentavalent salts of the metalloid arsenic. LSPR measurements indicate that the 3D hydrogel-nanoparticle coated sensors exhibited a higher sensitivity than that of the 2D AuNPs decorated sensors. Binding of As(III) to ArsA is greatly facilitated by the presence of magnesium ion and ATP.

Chapter 3 addresses the specific aim 2. It describes the sensing platform upgrade for biomarker study from living cell model. A SPR biosensor which is capable of monitoring proteomic biomarker secretion from living cells is reported here. The sensing strategy is based on the integration of a mini cell culture system within the traditional SPR sensing platform. SKOV-3 cells were incubated in the gelatin coated SPR flow chamber during the experiment. VEGF secretion from living SKOV-3 ovarian cancer cells was measured for concept demonstration. Fluorescent imaging was utilized to demonstrate cell viability in the SPR flow chamber. Rapid VEGF secretion from SKOV3 cells induced by $\mathrm{Ca}^{2+}$ ionophore was successfully monitored. The amount of VEGF released from SKOV-3 cells was determined by VEGF calibration curve. Cell number study reveals the relationship between SKOV-3 cell number and VEGF secretion amount, which helps to provide a strategy for accurate prediction of carcinoma cell number. By 
modifying the surface functionalization of the SPR assay, this biosensor might open up new horizons for detection and analysis of biomarker from living cells and tissue for different diseases.

Chapter 4 addresses the specific aim 3. It involves the evaluation of drug regulation on VEGF binding with living cell model. VEGF is one of the most important and best documented angiogenic factors, which is involved in the instigation of various types of tumor. VEGF-VEGFR binding process is the key point of angiogenesis, which is also the target of many angiogenesis inhibition therapy strategies. The purpose of this study was to construct a biomimic system of VEGF-VEGFR signaling pathway based on the platform described in chapter 3 for drug regulation study. Bevacizumab is a humanized anti-VEGF monoclonal antibody which is a FDA approved medicine for cancer therapy by regulating VEGF-VEGFR binding interaction. It has been proven to be clinically effect on angiogenesis inhibition. In this study, we employed bevacizumab as a model to demonstrate and evaluate our biomimic system for drug regulation study. Binding affinity and kinetics were studied for VEGF-VEGFR and VEGR-bevacizumab interactions. Results indicate that bevacizumab has higher binding affinity and association rate constant towards VEGF comparing with VEGFR. Biomimic study of bevacizumab regulation to the VEGF-VEGFR signaling pathway of living SKOV-3 cells shows successful blockage of the VEGF-VEGFR binding and activation process. A similar protocol can be used to evaluate drug regulation mechanisms in other disease models. 


\subsection{Future Work}

The LSPR biosensors developed for the arsenic detoxification pathway study will be further employed for the study of other proteins encoded by the ars operon. Binding kinetics of ArsA-As(III) interaction under the combined effect of ArsD and MgATP can be further investigated. Based on previous publication, the ars operon is also able to detoxify organic arsenic components. The LSPR biosensors could be useful for the study of interactions between ars proteins and organic arsenic components.

The signaling pathway biomimic system is capable of living cell studies, but it is lack of long term study capability. A temperature and $\mathrm{CO}_{2}$ controled mini cell culture system will be intergrated to the SPR biomimic systems to achieve long term real-time

measurement. Regulations by other medicine on the VEGF-VEGFR signaling pathway can also be investigated in the future. The SPR biosensors will also be tested for their sensitivity, specificity, reproducibility, etc. on the detection of biomarkers in body fluid from real patients. We have demonstrated the detection and kinetics studies on proteins using SPR. It will be very interesting to test the ability of detecting other types of cellular secretion (DNA, RNA, exosomes, etc.) on this sensing platform. The most promising potential of SPR in medical applications is early diagnosis. However, as we mentioned in chapter 1, SPR instruments are expensive and lack of portability. Research efforts should be put onto the development of cheaper, smaller and lighter SPR sensing platforms to make this technology more accessable for early diagnosis. 


\section{REFERENCES}

1. Turner, A. P. F., Biochemistry - Biosensors sense and sensitivity. Science 2000, (290), 1315-1317.

2. Ligler, F. S., Perspective on Optical Biosensors and Integrated Sensor Systems. Anal. Chem. 2009, (81), 519-526.

3. Karlsson, R.; Michaelsson, A.; Mattsson, L., KINETIC-ANALYSIS OF MONOCLONAL ANTIBODY-ANTIGEN INTERACTIONS WITH A NEW BIOSENSOR BASED ANALYTICAL SYSTEM. J. Immunol. Methods 1991, (145), 229-240.

4. Katz, E.; Willner, I., Probing biomolecular interactions at conductive and semiconductive surfaces by impedance spectroscopy: Routes to impedimetric immunosensors, DNA-Sensors, and enzyme biosensors. Electroanalysis 2003, (15), 913947.

5. Bousse, L., Whole cell biosensors. Sens. Actuator B-Chem. 1996, (34), 270-275.

6. Clark, L. C.; Lyons, C., ELECTRODE SYSTEMS FOR CONTINUOUS MONITORING IN CARDIOVASCULAR SURGERY. Annals of the New York Academy of Sciences 1962, (102), 29-45.

7. Sharma, A.; Rogers, K. R., BIOSENSORS. Meas. Sci. Technol. 1994, (5), 461472.

8. Homola, J.; Yee, S. S.; Gauglitz, G., Surface plasmon resonance sensors: review. Sens. Actuator B-Chem. 1999, (54), 3-15.

9. Homola, J.; Yee, S. S.; Gauglitz, G., Surface plasmon resonance sensors: review. Sensors and Actuators B: Chemical 1999, (54), 3-15.

10. Leatherbarrow, R. J.; Edwards, P. R., Analysis of molecular recognition using optical biosensors. Curr. Opin. Chem. Biol. 1999, (3), 544-547.

11. Cooper, M. A., Optical biosensors in drug discovery. Nat. Rev. Drug Discov. 2002, (1), 515-528.

12. Ince, R.; Narayanaswamy, R., Analysis of the performance of interferometry, surface plasmon resonance and luminescence as biosensors and chemosensors. Anal. Chim. Acta 2006, (569), 1-20.

13. Schuck, P., Use of surface plasmon resonance to probe the equilibrium and dynamic aspects of interactions between biological macromolecules. Annu. Rev. Biophys. Biomolec. Struct. 1997, (26), 541-566. 
14. Kawaguchi, T.; Shankaran, D. R.; Kim, S. J.; Matsumoto, K.; Toko, K.; Miura, N., Surface plasmon resonance immunosensor using Au nanoparticle for detection of TNT. Sens. Actuator B-Chem. 2008, (133), 467-472.

15. Kim, S. A.; Byun, K. M.; Kim, K.; Jang, S. M.; Ma, K.; Oh, Y.; Kim, D.; Kim, S. G.; Shuler, M. L.; Kim, S. J., Surface-enhanced localized surface plasmon resonance biosensing of avian influenza DNA hybridization using subwavelength metallic nanoarrays. Nanotechnology 2010, (21).

16. Fang, Y., Non-invasive optical biosensor for probing cell signaling. Sensors 2007, (7), 2316-2329.

17. Knoll, W.; Frank, C. W.; Heibel, C.; Naumann, R.; Offenhäusser, A.; Rühe, J.; Schmidt, E. K.; Shen, W. W.; Sinner, A., Functional tethered lipid bilayers. Reviews in Molecular Biotechnology 2000, (74), 137-158.

18. Balasubramanian, S.; Sorokulova, I. B.; Vodyanoy, V. J.; Simonian, A. L., Lytic phage as a specific and selective probe for detection of Staphylococcus aureus - A surface plasmon resonance spectroscopic study. Biosens. Bioelectron. 2007, (22), 948-955.

19. Li, Y.; Lee, H. J.; Corn, R. M., Detection of Protein Biomarkers Using RNA Aptamer Microarrays and Enzymatically Amplified Surface Plasmon Resonance Imaging. Anal. Chem. 2007, (79), 1082-1088.

20. Yu, L. L.; Wu, X. M.; Cheng, Z. Y.; Lee, C. V.; LeCouter, J.; Campa, C.; Fuh, G.; Lowman, H.; Ferrara, N., Interaction between bevacizumab and murine VEGF-A: A reassessment. Invest. Ophthalmol. Vis. Sci. 2008, (49), 522-527.

21. Kyo, M.; Usui-Aoki, K.; Koga, H., Label-Free Detection of Proteins in Crude Cell Lysate with Antibody Arrays by a Surface Plasmon Resonance Imaging Technique. Anal. Chem. 2005, (77), 7115-7121.

22. Vostiar, I.; Tkac, J.; Mandenius, C.-F., Intracellular monitoring of superoxide dismutase expression in an Escherichia coli fed-batch cultivation using on-line disruption with at-line surface plasmon resonance detection. Anal. Biochem. 2005, (342), 152-159.

23. Fujii, E.; Koike, T.; Nakamura, K.; Sasaki, S.; Kurihara, K.; Citterio, D.; Iwasaki, Y.; Niwa, O.; Suzuki, K., Application of an absorption-based surface plasmon resonance principle to the development of SPR ammonium ion and enzyme sensors. Anal. Chem. 2002, (74), 6106-6110.

24. Fang, S. P.; Lee, H. J.; Wark, A. W.; Corn, R. M., Attomole microarray detection of MicroRNAs by nanoparticle-amplified SPR imaging measurements of surface polyadenylation reactions. J. Am. Chem. Soc. 2006, (128), 14044-14046. 
25. Qin, J.; Fu, H. L.; Ye, J.; Bencze, K. Z.; Stemmler, T. L.; Rawlings, D. E.; Rosen, B. P., Convergent evolution of a new arsenic binding site in the ArsR/SmtB family of metalloregulators. J. Biol. Chem. 2007, (282), 34346-34355.

26. Zangar, R. C.; Daly, D. S.; White, A. M., ELISA microarray technology as a high-throughput system for cancer biomarker validation. Expert Rev. Proteomics 2006, (3), 37-44.

27. Ye, B.; Cramer, D. W.; Skates, S. J.; Gygi, S. P.; Pratomo, V.; Fu, L. F.; Horick, N. K.; Licklider, L. J.; Schorge, J. O.; Berkowitz, R. S.; Mok, S. C., Haptoglobin-alpha subunit as potential serum biomarker in ovarian cancer: Identification and characterization using proteomic profiling and mass spectrometry. Clin. Cancer Res. 2003, (9), 2904-2911.

28. Haes, A. J.; Van Duyne, R. P., Nanoscale optical biosensors based on localized surface plasmon resonance spectroscopy. 2003, 47-58.

29. Senaratne, W.; Andruzzi, L.; Ober, C. K., Self-assembled monolayers and polymer brushes in biotechnology: Current applications and future perspectives. Biomacromolecules 2005, (6), 2427-2448.

30. Luzinov, I.; Minko, S.; Tsukruk, V. V., Adaptive and responsive surfaces through controlled reorganization of interfacial polymer layers. Progress in Polymer Science 2004, (29), 635-698.

31. Schild, H. G., POLY (N-ISOPROPYLACRYLAMIDE) - EXPERIMENT, THEORY AND APPLICATION. Progress in Polymer Science 1992, (17), 163-249.

32. Pelton, R., Temperature-sensitive aqueous microgels. Adv. Colloid Interface Sci. 2000, (85), 1-33.

33. Bussemer, T.; Otto, I.; Bodmeier, R., Pulsatile drug-delivery systems. Crit. Rev. Ther. Drug Carr. Syst. 2001, (18), 433-458.

34. Vernon, B.; Kim, S. W.; Bae, Y. H., Thermoreversible copolymer gels for extracellular matrix. J. Biomed. Mater. Res. 2000, (51), 69-79.

35. Lindqvist, J.; Nystrom, D.; Ostmark, E.; Antoni, P.; Carlmark, A.; Johansson, M.; Hult, A.; Malmstrom, E., Intelligent dual-responsive cellulose surfaces via surfaceinitiated ATRP. Biomacromolecules 2008, (9), 2139-2145.

36. Jovanovic, J.; Adnadjevic, B.; Kostic, A., The effects of the $\mathrm{pH}$ value of the swelling medium on the kinetics of the swelling of a poly(acrylic acid) hydrogel. Journal of Applied Polymer Science 2010, (116), 1036-1043. 
37. Arizaga, A.; Ibarz, G.; Piñol, R., Stimuli-responsive poly(4-vinyl pyridine) hydrogel nanoparticles: Synthesis by nanoprecipitation and swelling behavior. $J$ Colloid Interf Sci 2010, (348), 668-672.

38. Bissen, M.; Frimmel, F. H., Arsenic - a Review. Part I: Occurrence, Toxicity, Speciation, Mobility. Acta hydrochimica et hydrobiologica 2003, (31), 9-18.

39. Nickson, R.; McArthur, J.; Burgess, W.; Ahmed, K. M.; Ravenscroft, P.; Rahman, M., Arsenic poisoning of Bangladesh groundwater. Nature 1998, (395), 338-338.

40. Smedley, P. L.; Kinniburgh, D. G., A review of the source, behaviour and distribution of arsenic in natural waters. Appl. Geochem. 2002, (17), 517-568.

41. Markiewicz, D. A.; Schultz, D. J.; Haas, J. A.; Harris, E. E. R.; Fox, K. R.; Glick, J. H.; Solin, L. J., The effects of sequence and type of chemotherapy and radiation therapy on cosmesis and complications after breast conservation therapy. Int. J. Radiat. Oncol. Biol. Phys. 1996, (35), 661-668.

42. Yoo, H. S.; Lee, K. H.; Oh, J. E.; Park, T. G., In vitro and in vivo anti-tumor activities of nanoparticles based on doxorubicin-PLGA conjugates. J. Control. Release 2000, (68), 419-431.

43. Folkman, J., ANGIOGENESIS IN CANCER, VASCULAR, RHEUMATOID AND OTHER DISEASE. Nat. Med. 1995, (1), 27-31.

44. Hanahan, D.; Weinberg, R. A., The Hallmarks of Cancer. Cell 2000, (100), 57-70.

45. Hanahan, D.; Weinberg, R. A., Hallmarks of Cancer: The Next Generation. Cell 2011, (144), 646-674.

46. Saaristo, A.; Karpanen, T.; Alitalo, K., Mechanisms of angiogenesis and their use in the inhibition of tumor growth and metastasis. Oncogene 2000, (19), 6122-6129.

47. Lee, H. J.; Nedelkov, D.; Corn, R. M., Surface plasmon resonance imaging measurements of antibody arrays for the multiplexed detection of low molecular weight protein biomarkers. Anal. Chem. 2006, (78), 6504-6510.

48. Natsume, T.; Nakayama, H.; Jansson, Ö.; Isobe, T.; Takio, K.; Mikoshiba, K., Combination of Biomolecular Interaction Analysis and Mass Spectrometric Amino Acid Sequencing. Anal. Chem. 2000, (72), 4193-4198.

49. Quinn, J. G.; O'Neill, S.; Doyle, A.; McAtamney, C.; Diamond, D.; MacCraith, B. D.; O'Kennedy, R., Development and application of surface plasmon resonance-based biosensors for the detection of cell-ligand interactions. Anal. Biochem. 2000, (281), 135143. 
50. Gao, Y.; Wolf, L. K.; Georgiadis, R. M., Secondary structure effects on DNA hybridization kinetics: a solution versus surface comparison. Nucleic Acids Res. 2006, (34), 3370-3377.

51. Cooper, M. A., Label-free screening of bio-molecular interactions. Anal. Bioanal. Chem. 2003, (377), 834-842.

52. Shan, X. N.; Huang, X. P.; Foley, K. J.; Zhang, P. M.; Chen, K. P.; Wang, S. P.; Tao, N. J., Measuring Surface Charge Density and Particle Height Using Surface Plasmon Resonance Technique. Anal. Chem. 2010, (82), 234-240.

53. Beyene, H. T.; Chakravadhanula, V. S. K.; Hanisch, C.; Elbahri, M.; Strunskus, T.; Zaporojtchenko, V.; Kienle, L.; Faupel, F., Preparation and plasmonic properties of polymer-based composites containing Ag-Au alloy nanoparticles produced by vapor phase co-deposition. J. Mater. Sci. 2010, (45), 5865-5871.

54. Jung, L. S.; Campbell, C. T.; Chinowsky, T. M.; Mar, M. N.; Yee, S. S., Quantitative interpretation of the response of surface plasmon resonance sensors to adsorbed films. Langmuir 1998, (14), 5636-5648.

55. Link, S.; El-Sayed, M. A., Spectral Properties and Relaxation Dynamics of Surface Plasmon Electronic Oscillations in Gold and Silver Nanodots and Nanorods. The Journal of Physical Chemistry B 1999, (103), 8410-8426.

56. Waswa, J.; Irudayaraj, J.; DebRoy, C., Direct detection of E-Coli O157 : H7 in selected food systems by a surface plasmon resonance biosensor. LWT-Food Sci. Technol. 2007, (40), 187-192.

57. Waswa, J. W.; Debroy, C.; Irudayaraj, J., RAPID DETECTION OF SALMONELLA ENTERITIDIS AND ESCHERICHIA COLI USING SURFACE PLASMON RESONANCE BIOSENSOR. Journal of Food Process Engineering 2006, (29), 373-385.

58. Liu, Y.; Dong, Y.; Jauw, J.; Linman, M. J.; Cheng, Q., Highly Sensitive Detection of Protein Toxins by Surface Plasmon Resonance with Biotinylation-Based Inline Atom Transfer Radical Polymerization Amplification. Anal. Chem. 2010, (82), 3679-3685.

59. Zhang, X. Z.; Yang, Y. Y.; Wang, F. J.; Chung, T. S., Thermosensitive poly(Nisopropylacrylamide-co-acrylic acid) hydrogels with expanded network structures and improved oscillating swelling-deswelling properties. Langmuir 2002, (18), 2013-2018.

60. Chang, Y.; Chen, W. Y.; Yandi, W.; Shih, Y. J.; Chu, W. L.; Liu, Y. L.; Chu, C. W.; Ruaan, R. C.; Higuchi, A., Dual-Thermoresponsive Phase Behavior of Blood Compatible Zwitterionic Copolymers Containing Nonionic Poly(N-isopropyl acrylamide). Biomacromolecules 2009, (10), 2092-2100. 
61. Ehrentreich-Forster, E.; Scheller, F. W.; Bier, F. F., Detection of progesterone in whole blood samples. Biosens. Bioelectron. 2003, (18), 375-380.

62. Abernathy, C. O.; Thomas, D. J.; Calderon, R. L., Health effects and risk assessment of arsenic. J. Nutr. 2003, (133), 1536S-1538S.

63. Chen, Y. X.; Rosen, B. P., Metalloregulatory properties of the ArsD repressor. $J$. Biol. Chem. 1997, (272), 14257-14262.

64. Sanfrancisco, M. J. D.; Hope, C. L.; Owolabi, J. B.; Tisa, L. S.; Rosen, B. P., IDENTIFICATION OF THE METALLOREGULATORY ELEMENT OF THE PLASMID-ENCODED ARSENICAL RESISTANCE OPERON. Nucleic Acids Res. 1990, (18), 619-624.

65. Wu, J.; Rosen, B. P., The arsD gene encodes a second trans-acting regulatory protein of the plasmid-encoded arsenical resistance operon. Molecular Microbiology 1993, (8), 615-623.

66. Lin, Y. F.; Walmsley, A. R.; Rosen, B. P., An arsenic metallochaperone for an arsenic detoxification pump. Proc. Natl. Acad. Sci. U. S. A. 2006, (103), 15617-15622.

67. Chen, C. M.; Misra, T. K.; Silver, S.; Rosen, B. P., NUCLEOTIDE-SEQUENCE OF THE STRUCTURAL GENES FOR AN ANION PUMP - THE PLASMIDENCODED ARSENICAL RESISTANCE OPERON. J. Biol. Chem. 1986, (261), 50305038.

68. Forzani, E. S.; Foley, K.; Westerhoff, P.; Tao, N. J., Detection of arsenic in groundwater using a surface plasmon resonance sensor. Sens. Actuator B-Chem. 2007, (123), 82-88.

69. Rich, R. L.; Myszka, D. G., Advances in surface plasmon resonance biosensor analysis. Current Opinion in Biotechnology 2000, (11), 54-61.

70. Zhou, T. Q.; Rosen, B. P., Tryptophan fluorescence reports nucleotide-induced conformational changes in a domain of the ArsA ATPase. J. Biol. Chem. 1997, (272), 19731-19737.

71. Bradford, M. M., A rapid and sensitive method for the quantitation of microgram quantities of protein utilizing the principle of protein-dye binding. Anal. Biochem. 1976, (72), 248-254.

72. Gill, S. C.; von Hippel, P. H., Calculation of protein extinction coefficients from amino acid sequence data. Anal. Biochem. 1989, (182), 319-326.

73. McFarland, A. D.; Haynes, C. L.; Mirkin, C. A.; Van Duyne, R. P.; Godwin, H. A., Color My Nanoworld. Journal of Chemical Education 2004, (81), 544A. 
74. Pan, Y. V.; Wesley, R. A.; Luginbuhl, R.; Denton, D. D.; Ratner, B. D., Plasma Polymerized N-Isopropylacrylamide: Synthesis and Characterization of a Smart Thermally Responsive Coating. Biomacromolecules 2000, (2), 32-36.

75. Hirata, I.; Okazaki, M.; Iwata, H., Simple method for preparation of ultra-thin poly (N-isopropylacrylamide) hydrogel layers and characterization of their thermoresponsive properties. Polymer 2004, (45), 5569-5578.

76. Yang, J. B.; Rawat, S.; Stemmler, T. L.; Rosen, B. P., Arsenic Binding and Transfer by the ArsD As(III) Metallochaperone. Biochemistry 2010, (49), 3658-3666.

77. Lin, Y. F.; Yang, J. B.; Rosen, B. P., ArsD residues Cys(12), Cys(13), and Cys(18) form an As(III)-binding site required for arsenic metallochaperone activity. J. Biol. Chem. 2007, (282), 16783-16791.

78. Zhou, T. Q.; Radaev, S.; Rosen, B. P.; Gatti, D. L., Structure of the ArsA ATPase: the catalytic subunit of a heavy metal resistance pump. Embo J. 2000, (19), 4838-4845.

79. Walmsley, A. R.; Zhou, T. Q.; Borges-Walmsley, M. I.; Rosen, B. P., The ATPase mechanism of ArsA, the catalytic subunit of the arsenite pump. J. Biol. Chem. 1999, (274), 16153-16161.

80. Ly, N.; Foley, K.; Tao, N. J., Integrated label-free protein detection and separation in real time using confined surface plasmon resonance imaging. Anal. Chem. 2007, (79), 2546-2551.

81. Taylor, A. D.; Ladd, J.; Yu, Q.; Chen, S.; Homola, J.; Jiang, S., Quantitative and simultaneous detection of four foodborne bacterial pathogens with a multi-channel SPR sensor. Biosensors and Bioelectronics 2006, (22), 752-758.

82. Su, X.; Wu, Y.-J.; Knoll, W., Comparison of surface plasmon resonance spectroscopy and quartz crystal microbalance techniques for studying DNA assembly and hybridization. Biosensors and Bioelectronics 2005, (21), 719-726.

83. Matsumoto, K.; Torimaru, A.; Ishitobi, S.; Sakai, T.; Ishikawa, H.; Toko, K.; Miura, N.; Imato, T., Preparation and characterization of a polyclonal antibody from rabbit for detection of trinitrotoluene by a surface plasmon resonance biosensor. Talanta 2005, (68), 305-311.

84. Forzani, E. S.; Zhang, H. Q.; Chen, W.; Tao, N. J., Detection of heavy metal ions in drinking water using a high-resolution differential surface plasmon resonance sensor. Environ. Sci. Technol. 2005, (39), 1257-1262.

85. Huang, X.; Gottstein, C.; Brekken, R. A.; Thorpe, P. E., Expression of Soluble VEGF Receptor 2 and Characterization of Its Binding by Surface Plasmon Resonance. Biochemical and Biophysical Research Communications 1998, (252), 643-648. 
86. von Tiedemann, B.; Bilitewski, U., Characterization of the vascular endothelial growth factor-receptor interaction and determination of the recombinant protein by an optical receptor sensor. Biosensors and Bioelectronics 2002, (17), 983-991.

87. Reddy, P. J.; Sadhu, S.; Ray, S.; Srivastava, S., Cancer Biomarker Detection by Surface Plasmon Resonance Biosensors. Clinics in Laboratory Medicine 2012, (32), 4772.

88. Altintas, Z.; Uludag, Y.; Gurbuz, Y.; Tothill, I. E., Surface plasmon resonance based immunosensor for the detection of the cancer biomarker carcinoembryonic antigen. Talanta 2011, (86), 377-383.

89. Presta, L. G.; Chen, H.; Oconnor, S. J.; Chisholm, V.; Meng, Y. G.; Krummen, L.; Winkler, M.; Ferrara, N., Humanization of an anti-vascular endothelial growth factor monoclonal antibody for the therapy of solid tumors and other disorders. Cancer Res. 1997, (57), 4593-4599.

90. Ferrara, N.; Gerber, H.-P.; LeCouter, J., The biology of VEGF and its receptors. Nat Med 2003, (9), 669-676.

91. Su, X. D.; Wu, Y. J.; Robelek, R.; Knoll, W., Surface plasmon resonance spectroscopy and quartz crystal microbalance study of streptavidin film structure effects on biotinylated DNA assembly and target DNA hybridization. Langmuir 2005, (21), 348353.

92. Oh, B.-K.; Kim, Y.-K.; Park, K. W.; Lee, W. H.; Choi, J.-W., Surface plasmon resonance immunosensor for the detection of Salmonella typhimurium. Biosensors and Bioelectronics 2004, (19), 1497-1504.

93. Lei, T.; Srinivasan, S.; Tang, Y.; Manchanda, R.; Nagesetti, A.; FernandezFernandez, A.; McGoron, A. J., Comparing cellular uptake and cytotoxicity of targeted drug carriers in cancer cell lines with different drug resistance mechanisms. Nanomedicine: Nanotechnology, Biology and Medicine 2011, (7), 324-332.

94. Mesiano, S.; Ferrara, N.; Jaffe, R. B., Role of Vascular Endothelial Growth Factor in Ovarian Cancer: Inhibition of Ascites Formation by Immunoneutralization. The American Journal of Pathology 1998, (153), 1249-1256.

95. Siegel, R.; Naishadham, D.; Jemal, A., Cancer statistics, 2012. CA: A Cancer Journal for Clinicians 2012, (62), 10-29.

96. Sherr, C. J., Cancer cell cycles. Science 1996, (274), 1672-1677.

97. Reya, T.; Morrison, S. J.; Clarke, M. F.; Weissman, I. L., Stem cells, cancer, and cancer stem cells. Nature 2001, (414), 105-111. 
98. Heng, H. H. Q.; Stevens, J. B.; Bremer, S. W.; Ye, K. J.; Liu, G.; Ye, C. J., The Evolutionary Mechanism of Cancer. J. Cell. Biochem. 2010, (109), 1072-1084.

99. Hermann, P. C.; Bhaskar, S.; Cioffi, M.; Heeschen, C., Cancer stem cells in solid tumors. Semin. Cancer Biol. 2010, (20), 77-84.

100. Golub, T. R.; Slonim, D. K.; Tamayo, P.; Huard, C.; Gaasenbeek, M.; Mesirov, J. P.; Coller, H.; Loh, M. L.; Downing, J. R.; Caligiuri, M. A.; Bloomfield, C. D.; Lander, E. S., Molecular classification of cancer: Class discovery and class prediction by gene expression monitoring. Science 1999, (286), 531-537.

101. Kuttesch, J. F.; Wexler, L. H.; Marcus, R. B.; Fairclough, D.; WeaverMcClure, L.; White, M.; Mao, L.; Delaney, T. F.; Pratt, C. B.; Horowitz, M. E.; Kun, L. E., Second malignancies after Ewing's sarcoma: Radiation dose-dependency of secondary sarcomas. J. Clin. Oncol. 1996, (14), 2818-2825.

102. Crosignani, P.; Demicheli, V.; Fontana, A.; Funto, I.; Masala, G.; Miligi, L.; Nanni, O.; Pisani, P.; Ramazzotti, V.; Rodella, S.; Costantini, A. S.; Stagnaro, E.; Tosi, P.; Tumino, R.; Vigano, C.; Vineis, P., Incidence and time trends for lymphomas, leukemias and myelomas: Hypothesis generation. Leuk. Res. 1996, (20), 285-290.

103. Weidner, N.; Semple, J. P.; Welch, W. R.; Folkman, J., TUMOR ANGIOGENESIS AND METASTASIS - CORRELATION IN INVASIVE BREASTCARCINOMA. N. Engl. J. Med. 1991, (324), 1-8.

104. Folkman, J., WHAT IS THE EVIDENCE THAT TUMORS ARE ANGIOGENESIS DEPENDENT. J. Natl. Cancer Inst. 1990, (82), 4-6.

105. Risau, W., Mechanisms of angiogenesis. Nature 1997, (386), 671-674.

106. Hanahan, D.; Folkman, J., Patterns and emerging mechanisms of the angiogenic switch during tumorigenesis. Cell 1996, (86), 353-364.

107. Folkman, J.; Shing, Y., ANGIOGENESIS. J. Biol. Chem. 1992, (267), 1093110934.

108. Carmeliet, P., Angiogenesis in health and disease. Nat. Med. 2003, (9), 653-660.

109. Carmeliet, P., Mechanisms of angiogenesis and arteriogenesis. Nat. Med. 2000, (6), 389-395.

110. Carmeliet, P., Angiogenesis in life, disease and medicine. Nature 2005, (438), 932-936.

111. Carmeliet, P.; Jain, R. K., Angiogenesis in cancer and other diseases. Nature 2000, (407), 249-257. 
112. Bergers, G.; Benjamin, L. E., Tumorigenesis and the angiogenic switch. Nat. Rev. Cancer 2003, (3), 401-410.

113. Yancopoulos, G. D.; Davis, S.; Gale, N. W.; Rudge, J. S.; Wiegand, S. J.; Holash, J., Vascular-specific growth factors and blood vessel formation. Nature 2000, (407), 242248.

114. Neufeld, G.; Cohen, T.; Gengrinovitch, S.; Poltorak, Z., Vascular endothelial growth factor (VEGF) and its receptors. Faseb J. 1999, (13), 9-22.

115. Hicklin, D. J.; Ellis, L. M., Role of the vascular endothelial growth factor pathway in tumor growth and angiogenesis. J. Clin. Oncol. 2005, (23), 1011-1027.

116. Dvorak, H. F., Vascular permeability factor/vascular endothelial growth factor: A critical cytokine in tumor angiogenesis and a potential target for diagnosis and therapy. $J$. Clin. Oncol. 2002, (20), 4368-4380.

117. Ferrara, N.; Kerbel, R. S., Angiogenesis as a therapeutic target. Nature 2005, (438), 967-974.

118. Prewett, M.; Huber, J.; Li, Y. W.; Santiago, A.; O'Connor, W.; King, K.; Overholser, J.; Hooper, A.; Pytowski, B.; Witte, L.; Bohlen, P.; Hicklin, D. J., Antivascular endothelial growth factor receptor (fetal liver kinase 1) monoclonal antibody inhibits tumor angiogenesis and growth of several mouse and human tumors. Cancer Res. 1999, (59), 5209-5218.

119. Ferrara, N.; Hillan, K. J.; Gerber, H. P.; Novotny, W., Discovery and development of bevacizumab, an anti-VEGF antibody for treating cancer. Nat. Rev. Drug Discov. 2004, (3), 391-400.

120. Willett, C. G.; Boucher, Y.; di Tomaso, E.; Duda, D. G.; Munn, L. L.; Tong, R. T.; Chung, D. C.; Sahani, D. V.; Kalva, S. P.; Kozin, S. V.; Mino, M.; Cohen, K. S.; Scadden, D. T.; Hartford, A. C.; Fischman, A. J.; Clark, J. W.; Ryan, D. P.; Zhu, A. X.; Blaszkowsky, L. S.; Chen, H. X.; Shellito, P. C.; Lauwers, G. Y.; Jain, R. K., Direct evidence that the VEGF-specific antibody bevacizumab has antivascular effects in human rectal cancer. Nat. Med. 2004, (10), 145-147.

121. Johnson, D. H.; Fehrenbacher, L.; Novotny, W. F.; Herbst, R. S.; Nemunaitis, J. J.; Jablons, D. M.; Langer, C. J.; DeVore, R. F.; Gaudreault, J.; Damico, L. A.; Holmgren, E.; Kabbinavar, F., Randomized phase II trial comparing bevacizumab plus carboplatin and paclitaxel with carboplatin and paclitaxel alone in previously untreated locally advanced or metastatic non-small-cell lung cancer. J. Clin. Oncol. 2004, (22), 2184-2191.

122. Miller, K. D.; Chap, L. I.; Holmes, F. A.; Cobleigh, M. A.; Marcom, P. K.; Fehrenbacher, L.; Dickler, M.; Overmoyer, B. A.; Reimann, J. D.; Sing, A. P.; Langmuir, V.; Rugo, H. S., Randomized phase III trial of capecitabine compared with bevacizumab 
plus capecitabine in patients with previously treated metastatic breast cancer. J. Clin. Oncol. 2005, (23), 792-799.

123. Saltz, L. B.; Clarke, S.; Diaz-Rubio, E.; Scheithauer, W.; Figer, A.; Wong, R.; Koski, S.; Lichinitser, M.; Yang, T. S.; Rivera, F.; Couture, F.; Sirzen, F.; Cassidy, J., Bevacizumab in combination with oxaliplatin-based chemotherapy as first-line therapy in metastatic colorectal cancer: A randomized phase III study. J. Clin. Oncol. 2008, (26), 2013-2019.

124. Friedman, H. S.; Prados, M. D.; Wen, P. Y.; Mikkelsen, T.; Schiff, D.; Abrey, L. E.; Yung, W. K. A.; Paleologos, N.; Nicholas, M. K.; Jensen, R.; Vredenburgh, J.; Huang, J.; Zheng, M. X.; Cloughesy, T., Bevacizumab Alone and in Combination With Irinotecan in Recurrent Glioblastoma. J. Clin. Oncol. 2009, (27), 4733-4740.

125. Tol, J.; Koopman, M.; Cats, A.; Rodenburg, C. J.; Creemers, G. J. M.; Schrama, J. G.; Erdkamp, F. L. G.; Vos, A. H.; van Groeningen, C. J.; Sinnige, H. A. M.; Richel, D. J.; Voest, E. E.; Dijkstra, J. R.; Vink-Borger, M. E.; Antonini, N. F.; Mol, L.; van Krieken, J.; Dalesio, O.; Punt, C. J. A., Chemotherapy, Bevacizumab, and Cetuximab in Metastatic Colorectal Cancer. N. Engl. J. Med. 2009, (360), 563-572.

126. McDermott, D. F.; George, D. J., Bevacizumab as a treatment option in advanced renal cell carcinoma: An analysis and interpretation of clinical trial data. Cancer Treat. Rev. 2010, (36), 216-223.

127. Patel, P. J.; Bunce, C.; Tufail, A.; Investigators, A. B. C. T., A randomised, double-masked phase III/IV study of the efficacy and safety of Avastin (R) (Bevacizumab) intravitreal injections compared to standard therapy in subjects with choroidal neovascularisation secondary to age-related macular degeneration: clinical trial design. Trials 2008, (9).

128. Yang, J. C.; Haworth, L.; Sherry, R. M.; Hwu, P.; Schwartzentruber, D. J.; Topalian, S. L.; Steinberg, S. M.; Chen, H. X.; Rosenberg, S. A., A randomized trial of bevacizumab, an anti-vascular endothelial growth factor antibody, for metastatic renal cancer. N. Engl. J. Med. 2003, (349), 427-434.

129. Michels, S.; Rosenfeld, P. J.; Puliafito, C. A.; Marcus, E. N.; Venkatraman, A. S., Systemic bevacizumab (Avastin) therapy for neovascular age-related macular degeneration - Twelve-week results of an uncontrolled open-label clinical study. Ophthalmology 2005, (112), 1035-1047.

130. Bock, F.; Onderka, J.; Dietrich, T.; Bachmann, B.; Kruse, F. E.; Paschke, M.; Zahn, G.; Cursiefen, C., Bevacizumab as a potent inhibitor of inflammatory corneal angiogenesis and lymphangiogenesis. Invest. Ophthalmol. Vis. Sci. 2007, (48), 2545-2552.

131. Klettner, A.; Roider, J., Comparison of bevacizumab, ranibizumab, and pegaptanib in vitro: Efficiency and possible additional pathways. Invest. Ophthalmol. Vis. Sci. 2008, (49), 4523-4527. 
132. Liu, C.; Lei, T.; Ino, K.; Matsue, T.; Tao, N.; Li, C.-Z., Real-time monitoring biomarker expression of carcinoma cells by surface plasmon resonance biosensors. Chemical Communications 2012, (48), 10389-10391.

133. Hsieh, C. H.; Kuo, W. T.; Huang, Y. C.; Huang, Y. Y., High-efficiency cell seeding method by relatively hydrophobic culture strategy. J. Biomed. Mater. Res. Part B 2011, (98B), 38-46.

134. Kramer, I.; Lipp, H. P., Bevacizumab, a humanized anti-angiogenic monoclonal antibody for the treatment of colorectal cancer. J. Clin. Pharm. Ther. 2007, (32), 1-14.

135. Edwards, P. R.; Leatherbarrow, R. J., Determination of Association Rate Constants by an Optical Biosensor Using Initial Rate Analysis. Anal. Biochem. 1997, (246), 1-6.

136. Nagengast, W. B.; de Vries, E. G.; Hospers, G. A.; Mulder, N. H.; de Jong, J. R.; Hollema, H.; Brouwers, A. H.; van Dongen, G. A.; Perk, L. R.; Lub-de Hooge, M. N., In vivo VEGF imaging with radiolabeled bevacizumab in a human ovarian tumor xenograft. J. Nucl. Med. 2007, (48), 1313-1319. 
VITA

CHANG LIU

Born, Beijing, P.R.China

2003-2007

B.S., Biomedical Engineering

Beijing JiaoTong University

Beijing, P.R.China

2007-present

Ph.D. Candidate, Biomedical Engineering

Expected Graduation: April, 2013

Florida International University

Miami, Florida

2011

Chinese Government Award for Outstanding Selffinanced Students Aboard $(\$ 5,000)$

2012

"Top-50 most cited articles" as published in Biosensors \& Bioelectronics (2010-2011):

Membraneless enzymatic biofuel cells based on graphene nanosheets

2012

Research article "Real-Time Monitoring Biomarker Expression of Carcinoma Cells by Surface Plasmon Resonance Biosensors" featured as inside cover on Chemical Communications, volume 48.

\section{PUBLICATIONS AND PRESENTATIONS}

S. Alwarappan, A. Erdem, C. Liu and C. Z. Li, "Probing the electrochemical properties of graphene nanosheets for biosensing applications", J. Phy. Chem. C, vol. 113, pp. 8853$8857,2009$.

B. Chen, Y. Xiao, C. Liu, C. Z. Li and F. Leng, "DNA linking number change induced by sequence-specific DNA-binding proteins", Nucleic Acids Res., vol. 38, pp. 3643-3654, 2010 .

S. Alwarappan, C. Liu, A. Kumar, and C. Z. Li, "Enzyme-doped graphene nanosheets for enhanced glucose biosensing", J. Phys. Chem. C, vol. 114, pp. 12920-12924, 2010.

C. Liu, S. Alwarappan, Z. Chen, X. Kong and C. Z. Li, "Membraneless enzymatic biofuel cells based on graphene nanosheets", Biosens. Bioelectron., vol. 25, pp. 1829-1833, 2010. 
E. Hondroulis, C. Liu and C. Z. Li, "Whole cell based electrical impedance sensing approach for a rapid nanotoxicity assay", Nanotechnology, vol. 21, 315103, 2010.

C. Liu and C. Z. Li, "Surface engineering of graphene-enzyme nano composites for miniaturized biofuel cell", IEEE Trans. Nanotechnol., vol 10, pp. 59-62, 2011.

C. Liu, V. Balsamo, D. Sun, M. Naja, X. Wang, B. Rosen, C. Z. Li, “A 3D localized surface plasmon resonance biosensor for the study of trivalent arsenic binding to the ArsA ATPase", Biosens. Bioelectron., vol 38, pp. 19-26, 2012.

C. Liu, T. Lei, I. Kosuke, T. Matsue, N. Tao and C. Z. Li, "Real-Time Monitoring Biomarker Expression of Carcinoma Cells by Surface Plasmon Resonance Biosensors", Chem. Commun., vol 48, pp. 10389-10391, 2012.

C. Liu, S. Alwarappan, and C. Z. Li, "Enzyme-Graphene nanoconjugation for biofuel cell applications", Nanoelectronic Devices for Defense \& Security Conference, Fort Lauderdale, Florida, 2009.

C. Liu, S. Alwarappan, and C. Z. Li, "Performance comparison of enzymatic biofuel cells using graphene nanosheets and carbon nanotube materials", Pittcon 2010, Orlando, Florida, 2010.

C. Liu, V. Balsamo, D. Sun, B. Rosen and C. Z. Li, "Enhanced Surface Plasmon Resonance Sensing of Arsenic-protein Interaction”, Pittcon 2011, Atlanta, Georgia, 2011.

C. Liu, S. Alwarappan, and C. Z. Li, "Carbon based nanomaterials for bioenergy devices" (Invited), the 4th Annual NanoScience Technology Symposium (NanoFlorida 2011), Miami, Florida, 2011.

P. Shah, S. Prabhulkar, C. Liu and C. Z. Li, "Miniaturized Biosensors for Biomarker Analysis at Single Cellular Level", the Miami 2012 Winter Symposium: Nanotechnology in Biomedicine (Nature Conference), Miami, Florida, 2012.

C. Liu and C. Z. Li, "In situ Study of Proteomic Cancer Biomarker Secretion", Pittcon 2012, Orlando, Florida, 2012.

C. Liu and C. Z. Li, "In situ Surface Plasmon Resonance Biosensor for Monitoring Biomarker Expression from Living Carcinoma Cells", the 5th Annual NanoScience Technology Symposium (NanoFlorida 2012), Tampa, Florida, 2012.

C. Liu, T. Matsue, and C. Z. Li, "Direct Observation of Vascular Endothelial Growth Factor Secretion from a Living Carcinoma Cell Model by Surface Plasmon Resonance", Biomedical Engineering Society 2012 Annual Meeting, Atlanta, Georgia, 2012. 